\title{
Silyl Radical Activation of Alkyl Halides in \\ Metallaphotoredox Catalysis: A Unique Pathway for Cross- Electrophile Coupling
}

Patricia Zhang, Chi “Chip" Le and David W. C. MacMillan*

Merck Center for Catalysis at Princeton University, Princeton, New Jersey 08544

\section{Supporting Information}




\section{Table of Contents}

1) General Information

2) Procedure for Optimization Studies

3) Procedure for Silane-Mediated Metallaphotoredox Alkyl-Aryl Cross-

Electrophile Coupling

4) Aryl Halide Scope

S7

5) Alkyl Halide Scope

S31

6) Cyclic Voltammetry Data

S46

7) Stern-Volmer Fluorescence Quenching Experiments

8) Procedure for Investigating Other Reductants (Table 3)

S48

9) References

S49

10) Spectral Data for Alkyl-Aryl Compounds

S50 


\section{1) General Information}

Commercial reagents were purified prior to use following the guidelines of Perrin and Armarego ${ }^{1}$. $\operatorname{Ir}[\mathrm{dF}(\mathrm{CF} 3)$ ppy $] 2$ (dtbbpy)PF6 was prepared using literature procedures ${ }^{2}$. Reagent grade dimethoxyethane was used for the alkyl-aryl cross-electrophile reactions. All other solvents were purified according to the method of Grubbs ${ }^{3}$. Organic solutions were concentrated under reduced pressure on a Büchi rotary evaporator using a water bath. Chromatographic purification of products was accomplished using forced-flow chromatography on silica gel (Fluka, 230-400 mesh) according to the method of Still ${ }^{4}$. Thin-layer chromatography (TLC) was performed on Silicycle $0.25 \mathrm{~mm}$ silica gel F-254 plates. Visualization of the developed chromatogram was performed by fluorescence quenching or $\mathrm{KMnO}_{4}$ stain. ${ }^{1} \mathrm{H} \mathrm{NMR}$ spectra were recorded on a Bruker UltraShield Plus Avance III $500 \mathrm{MHz}$ and are internally referenced to residual protic $\mathrm{CDCl} 3(\delta 7.26 \mathrm{ppm})$ and $\left(\mathrm{CD}_{3}\right)_{2} \mathrm{CO}$ signals $(\delta$ $2.05 \mathrm{ppm})$. Data for ${ }^{1} \mathrm{H}$ NMR are reported as follows: chemical shift $(\delta \mathrm{ppm})$, multiplicity $(\mathrm{s}=$ singlet, $\mathrm{d}=$ doublet, $\mathrm{t}=$ triplet, $\mathrm{q}=$ quartet, $\mathrm{m}=$ multiplet, $\mathrm{dd}=$ doublet of doublets, $\mathrm{dt}=$ doublet of triplets, $\mathrm{br}=$ broad), coupling constant $(\mathrm{Hz})$, and integration. ${ }^{13} \mathrm{C}$ NMR spectra were recorded on a Bruker UltraShield Plus Avance III $500 \mathrm{MHz}(125 \mathrm{MHz})$ and data are reported in terms of chemical shift relative to $\mathrm{CDCl} 3$ (77.16 ppm) or $\left(\mathrm{CD}_{3}\right)_{2} \mathrm{CO}$ (29.84 ppm and $\left.206.26 \mathrm{ppm}\right) .{ }^{19} \mathrm{~F}$ NMR spectra were recorded on a Bruker NanoBay $300 \mathrm{MHz}(282 \mathrm{MHz}$ ). IR spectra were recorded on a Perkin Elmer Spectrum 100 FTIR spectrometer and are reported in wavenumbers (cm-1). High Resolution Mass Spectra were obtained from the Princeton University Mass Spectral Facility. 


\section{2) Procedure for Optimization Studies}

To an $8 \mathrm{~mL}$ vial equipped with a stir bar was added photocatalyst $\operatorname{Ir}\left[\mathrm{dF}\left(\mathrm{CF}_{3}\right) \text { ppy }\right]_{2}(\mathrm{dtbbpy}) \mathrm{PF}_{6} \quad(2.5 \mathrm{mg}, 2.5 \mu \mathrm{mol}, 0.01$ equiv.), methyl 4-bromo benzoate (54 mg, $0.25 \mathrm{mmol}, 1$ equiv.), 4-bromotetrahydropyran ( $42 \mu \mathrm{L}, 0.375$ mmol, 1.5 equiv.), tris(trimethylsilyl)silane (77 $\mu \mathrm{L}, 0.25 \mathrm{mmol}, 1.0$ equiv), and anhydrous sodium carbonate (53 $\mathrm{mg}, 0.5 \mathrm{mmol}, 2$ equiv.). The vial was sealed and placed under nitrogen before $2 \mathrm{~mL}$ of solvent was added. To a separate vial was added $\mathrm{NiCl}_{2} \bullet$ glyme (2.8 mg, $0.013 \mathrm{mmol}, 0.05$ equiv.) and 4,4'-di-tert-butyl-2,2'-bipyridine (3.4 mg, $0.013 \mathrm{mmol}, 0.05$ equiv). The catalyst vial was sealed, purged with nitrogen then to it was added $1 \mathrm{~mL}$ of solvent. The precatalyst solution was sonicated or stirred for 5 minutes, after which, $0.1 \mathrm{~mL}$ of the solution $(0.5 \mathrm{~mol} \%$ catalyst, $1.25 \mu \mathrm{mol}$, 0.005 equv.) was syringed into the reaction vessel. The solution was degassed by sparging with nitrogen while stirring for 10 minutes before sealing with Parafilm. The reaction was stirred and irradiated with a $34 \mathrm{~W}$ blue LED lamp (7 cm away, with cooling fan to keep the reaction temperature at $25^{\circ} \mathrm{C}$ ) for 6 hours. The reaction was quenched by exposure to air. Mesitylene (internal standard, $35 \mu \mathrm{L}, 0.250 \mathrm{mmol}, 1.0$ equiv.) was added then the reaction mixture was analyzed by ${ }^{1} \mathrm{H}$ NMR. 


\section{3) Procedure for Silane-Mediated Metallaphotoredox Alkyl-Aryl Cross- Electrophile Coupling}

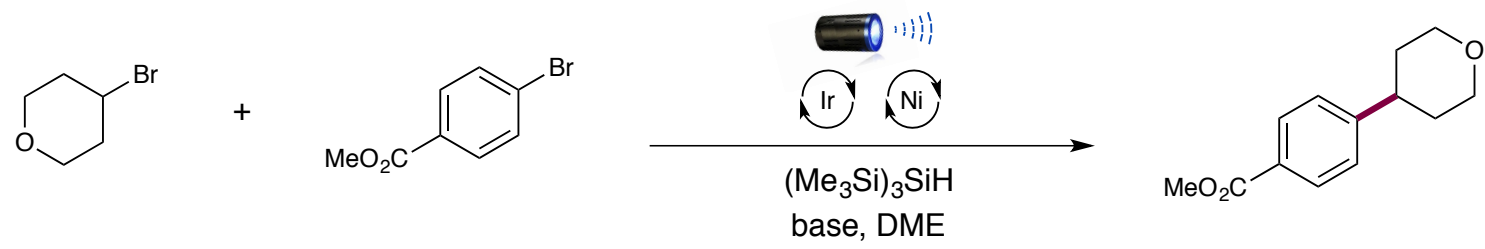

To an $8 \mathrm{~mL}$ vial equipped with a stir bar was added photocatalyst $\operatorname{Ir}\left[\mathrm{dF}\left(\mathrm{CF}_{3}\right) \text { ppy }\right]_{2}(\mathrm{dtbbpy}) \mathrm{PF}_{6}$ (5.6 $\mathrm{mg}, 5.0 \mu \mathrm{mol}, 0.01$ equiv.), methyl 4-bromo benzoate (108 mg, $0.5 \mathrm{mmol}, 1$ equiv.), 4-bromotetrahydropyran ( $84 \mu \mathrm{L}, 0.750$ mmol, 1.5 equiv.), tris(trimethylsilyl)silane ( $154 \mu \mathrm{L}, 0.5 \mathrm{mmol}, 1.0$ equiv), and anhydrous sodium carbonate (106 mg, $1.0 \mathrm{mmol}, 2$ equiv.). The vial was sealed and placed under nitrogen before $4 \mathrm{~mL}$ of DME was added. To a separate vial was added $\mathrm{NiCl}_{2} \bullet$ glyme (1 mg, 5 umol, 0.01 equiv.) and 4,4'-di-tert-butyl-2,2'-bipyridine (1.3 $\mathrm{mg}, 5 \mu \mathrm{mol}, 0.01$ equiv). The catalyst vial was sealed, purged with nitrogen then to it was added $2 \mathrm{~mL}$ of DME. The precatalyst solution was sonicated or stirred for 5 minutes, after which, $1 \mathrm{~mL}(0.5 \mathrm{~mol} \%$ catalyst, $2.5 \mu \mathrm{mol}, 0.005$ equv.) was syringed into the reaction vessel. The solution was degassed by sparging with nitrogen while stirring for 10 minutes before sealing with Parafilm. The reaction was stirred and irradiated with a $34 \mathrm{~W}$ blue LED lamp ( $7 \mathrm{~cm}$ away, with cooling fan to keep the reaction temperature at $25^{\circ} \mathrm{C}$ ) for 6 hours. The reaction was quenched by exposure to air and concentrated in vacuo. Purification by column chromatography (silica gel, 0 $60 \%$ EtOAc in hexanes) yielded the alkyl-aryl product.

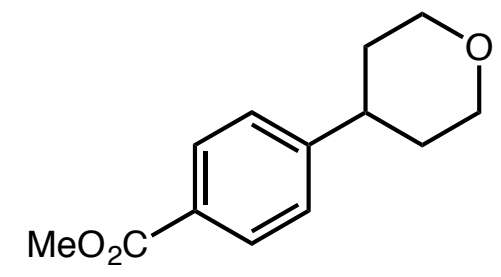

\section{methyl 4-(tetrahydro-2H-pyran-4-yl)benzoate}

Prepared following the general procedure outlined above using $\operatorname{Ir}\left[\mathrm{dF}_{(}\left(\mathrm{CF}_{3}\right) \text { ppy }\right]_{2}$ (dtbbpy)PF 6 (5.6 mg, $0.5 \mu \mathrm{mol}, 0.01$ equiv.), $\mathrm{NiCl}_{2} \bullet$ glyme (0.5 mg, $2.5 \mu \mathrm{mol}, 0.005$ equiv.), 4,4'-di-tert-butyl-2,2'-bipyridine ( $0.8 \mathrm{mg}, 3.0 \mu \mathrm{mol}, 0.006$ equiv, $\mathrm{Na}_{2} \mathrm{CO}_{3}(106.0$ 
mg, 1.00 mmol, 2.0 equiv.), TTMSS (154 $\mu \mathrm{L}, 0.50$ mmol, 1.0 equiv.), 4bromotetrahydro-2H-pyran ( $84 \mu \mathrm{L}, 0.75 \mathrm{mmol}, 1.5$ equiv.), methyl 4-bromobenzoate (108 mg, $0.50 \mathrm{mmol}, 1.0$ equiv.), and DME (5.0 mL). Purification by column chromatography yielded the pure product as a white solid $(87 \mathrm{mg}, 0.39 \mathrm{mmol}, 79 \%$ yield).

${ }^{1}$ H NMR (500 MHz, $\left.\mathbf{C D C l}_{3}\right) \delta 7.97(\mathrm{~d}, J=8.2 \mathrm{~Hz}, 2 \mathrm{H}), 7.29$ (d, $\left.J=8.2 \mathrm{~Hz}, 2 \mathrm{H}\right), 4.15$ $4.04(\mathrm{~m}, 2 \mathrm{H}), 3.91(\mathrm{~s}, 3 \mathrm{H}), 3.54(\mathrm{td}, J=11.6,2.5 \mathrm{~Hz}, 2 \mathrm{H}), 2.82(\mathrm{tt}, J=11.7,4.2 \mathrm{~Hz}, 1 \mathrm{H})$, $1.90-1.72(\mathrm{~m}, 4 \mathrm{H})$.

${ }^{13}$ C NMR (125 MHz, $\left.\mathbf{C D C l}_{3}\right)$ 8167.03, 151.08, 129.91, 128.30, 126.81, 77.28, 77.03, 76.77, 68.24, 52.06, 41.67, 33.60.

Spectroscopic data matches with previously reported data. ${ }^{5}$ 


\section{4) Aryl Halide Scope}

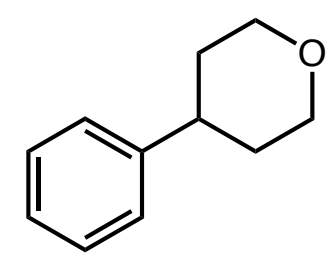

\section{4-phenyltetrahydro-2H-pyran (1)}

Prepared following the general procedure outlined above using $\operatorname{Ir}\left[\mathrm{dF}\left(\mathrm{CF}_{3}\right) \text { ppy }\right]_{2}$ (dtbbpy)PF 6 (5.6 mg, $5.0 \mu \mathrm{mol}, 0.01$ equiv.), $\mathrm{NiCl}_{2} \bullet$ glyme (0.5 mg, $2.5 \mu \mathrm{mol}, 0.005$ equiv.), 4,4'-di-tert-butyl-2,2'-bipyridine ( $0.8 \mathrm{mg}, 3.0 \mu \mathrm{mol}, 0.006$ equiv.), $\mathrm{Na}_{2} \mathrm{CO}_{3}$ (106.0 mg, $1.00 \mathrm{mmol}, 2.0$ equiv.), tris(triethylsilyl)silane $(211 \mu \mathrm{L}, 0.5 \mathrm{mmol}, 1.0$ equiv.), 4-bromotetrahydro-2H-pyran ( $84 \mu \mathrm{L}, 0.75 \mathrm{mmol}, 1.5$ equiv.), bromobenzene (52 $\mu \mathrm{L}, 0.5 \mathrm{mmol}, 1.0$ equiv.), and DME (5.0 mL). Purification by column chromatography yielded the pure product as a white solid (64 mg, $0.39 \mathrm{mmol}, 79 \%$ yield).

***Tris(triethylsilyl)silane was used for ease of purification. The reaction gave the same efficiency when tris(trimethylsilyl)silane was employed.***

${ }^{1}$ H NMR (500 MHz, CDCl $) \delta 7.37-7.30(\mathrm{~m}, 2 \mathrm{H}), 7.23(\mathrm{~m}, 3 \mathrm{H}), 4.16-4.02(\mathrm{~m}, 2 \mathrm{H})$, $3.54(\mathrm{td}, J=11.6,2.5 \mathrm{~Hz}, 2 \mathrm{H}), 2.76(\mathrm{tt}, J=11.7,4.2 \mathrm{~Hz}, 1 \mathrm{H}), 1.91-1.73(\mathrm{~m}, 4 \mathrm{H})$.

${ }^{13}$ C NMR (125 MHz, $\left.\mathbf{C D C l}_{3}\right) \delta 145.88,128.53,126.76,126.75,126.33,77.29,77.04$, $76.79,68.44,41.60,33.96$.

Spectroscopic data matches with previously reported data. ${ }^{6}$ 


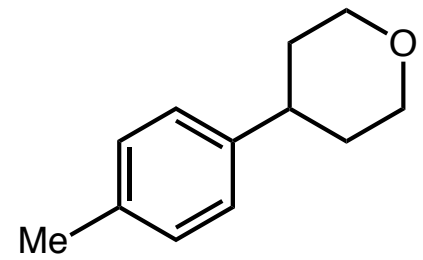

\section{4-(p-tolyl)tetrahydro-2H-pyran (2)}

Prepared following the general procedure outlined above using $\operatorname{Ir}\left[\mathrm{dF}\left(\mathrm{CF}_{3}\right) \text { ppy }\right]_{2}$ (dtbbpy)PF 6 (5.5 mg, $5.0 \mu \mathrm{mol}, 0.01$ equiv.), $\mathrm{NiCl}_{2} \bullet$ glyme (0.5 mg, $2.5 \mu \mathrm{mol}, 0.005$ equiv.), 4,4'-di-tert-butyl-2,2'-bipyridine ( $0.8 \mathrm{mg}, 3.0 \mu \mathrm{mol}, 0.006$ equiv.), $\mathrm{Na}_{2} \mathrm{CO}_{3}$ (106.0 mg, 1.00 mmol, 2.0 equiv.), TTMSS (154 $\mu \mathrm{L}, 0.50 \mathrm{mmol}, 1.0$ equiv.), 4bromotetrahydro-2H-pyran ( $84 \mu \mathrm{L}, 0.75 \mathrm{mmol}, 1.5$ equiv.), 4-bromotoluene $(86.0 \mathrm{mg}$, $0.50 \mathrm{mmol}, 1.0$ equiv.), and DME $(5.0 \mathrm{~mL})$. Purification by column chromatography yielded the pure product as a clear oil (72 $\mathrm{mg}, 0.41 \mathrm{mmol}, 82 \%$ yield).

${ }^{1}$ H NMR (500 MHz, CDCl 3 ) $\delta 7.13$ (s, 4H), 4.08 (ddt, $\left.J=11.7,4.5,1.1 \mathrm{~Hz}, 2 \mathrm{H}\right), 3.53$ (td, $J=11.6,2.5 \mathrm{~Hz}, 2 \mathrm{H}), 2.72$ (tt, $J=11.7,4.2 \mathrm{~Hz}, 1 \mathrm{H}), 2.33$ (s, 3H), $1.87-1.69$ (m, $4 \mathrm{H})$.

${ }^{13}$ C NMR (125 MHz, CDCl 3$) \delta 142.9,135.8,129.2,126.6,68.4,41.1,34.0,21.0$.

Spectroscopic data matches with previously reported data. ${ }^{7}$ 


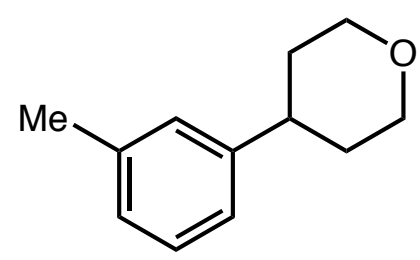

\section{4-(m-tolyl)tetrahydro-2H-pyran (3)}

Prepared following the general procedure outlined above using $\operatorname{Ir}\left[\mathrm{dF}\left(\mathrm{CF}_{3}\right) \text { ppy }\right]_{2}$ (dtbbpy)PF 6 (5.6 mg, $0.5 \mu \mathrm{mol}, 0.01$ equiv.), $\mathrm{NiCl}_{2} \bullet$ glyme (0.5 mg, $2.5 \mu \mathrm{mol}, 0.005$ equiv.), 4,4'-di-tert-butyl-2,2'-bipyridine ( $0.8 \mathrm{mg}, 3.0 \mu \mathrm{mol}, 0.006$ equiv, $\mathrm{Na}_{2} \mathrm{CO}_{3}$ (106.0 mg, 1.00 mmol, 2.0 equiv.), TTMSS (154 $\mu \mathrm{L}, 0.50$ mmol, 1.0 equiv.), 4bromotetrahydro-2H-pyran ( $84 \mu \mathrm{L}, 0.75 \mathrm{mmol}, 1.5$ equiv.), 1-bromo-3-methylbenzene (86 mg, $61 \mu \mathrm{L}, 0.5 \mathrm{mmol}, 1.0$ equiv.), and DME (5.0 mL). Purification by column chromatography yielded the pure product as a white crystalline solid $(71 \mathrm{mg}, 0.40 \mathrm{mmol}$, $80 \%$ yield).

${ }^{1}$ H NMR (500 MHz, $\left.\mathbf{C D C l}_{3}\right) \delta 7.25$ - 7.19 (m, 1H), 7.04 (d, $\left.J=8.3 \mathrm{~Hz}, 3 \mathrm{H}\right), 4.15-4.02$ $(\mathrm{m}, 2 \mathrm{H}), 3.53(\mathrm{td}, J=11.7,2.4 \mathrm{~Hz}, 2 \mathrm{H}), 2.72(\mathrm{tt}, J=11.8,4.1 \mathrm{~Hz}, 1 \mathrm{H}), 2.35(\mathrm{~s}, 3 \mathrm{H}), 1.90$ $-1.71(\mathrm{~m}, 4 \mathrm{H})$.

${ }^{13}$ C NMR (125 MHz, $\left.\mathbf{C D C l}_{3}\right) \delta 145.87,138.07,128.43,127.59,127.07,123.74,68.47$, $41.55,21.52$.

IR (film) $v_{\max } 3021,2931,2839,1607,1129,1086 \mathrm{~cm}^{-1}$.

HRMS (ESI-TOF) m/z calcd. for $\mathrm{C}_{12} \mathrm{H}_{17} \mathrm{O}\left([\mathrm{M}+\mathrm{H}]^{+}\right)$177.1274, found 177.1275. 


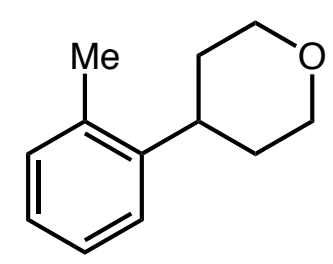

\section{4-(o-tolyl)tetrahydro-2H-pyran (4)}

Prepared following the general procedure outlined above using $\operatorname{Ir}\left[\mathrm{dF}_{(}\left(\mathrm{CF}_{3}\right) \text { ppy }\right]_{2}$ (dtbbpy)PF 6 (5.6 mg, $0.5 \mu \mathrm{mol}, 0.01$ equiv.), $\mathrm{NiCl}_{2} \bullet$ glyme (0.5 mg, $2.5 \mu \mathrm{mol}, 0.005$ equiv.), 4,4'-di-tert-butyl-2,2'-bipyridine (0.8 mg, $3.0 \mu \mathrm{mol}, 0.006$ equiv, $\mathrm{Na}_{2} \mathrm{CO}_{3}$ (106.0 mg, 1.00 mmol, 2.0 equizv.), TTMSS (154 $\mu \mathrm{L}, 0.50$ mmol, 1.0 equiv.), 4bromotetrahydro-2H-pyran ( $84 \mu \mathrm{L}, 0.75 \mathrm{mmol}, 1.5$ equiv.), 1-bromo-2-methylbenzene (86 mg, $60 \mu \mathrm{L}, 0.5 \mathrm{mmol}, 1.0$ equiv.), and DME (5.0 mL). Purification by column chromatography yielded the pure product as a white crystalline solid ( $74 \mathrm{mg}, 0.42 \mathrm{mmol}$, $83 \%$ yield).

${ }^{1}$ H NMR (500 MHz, CDCl $) \delta 7.25-7.08(\mathrm{~m}, 4 \mathrm{H}), 4.14-4.07(\mathrm{~m}, 3 \mathrm{H}), 3.56(\mathrm{td}, J=$ 11.8, 2.0 Hz, 3H), $2.98(\mathrm{tt}, J=11.9,3.6 \mathrm{~Hz}, 1 \mathrm{H}), 2.36$ (s, 3H), 1.83 (dtd, $J=13.5,12.0$, $4.4 \mathrm{~Hz}, 2 \mathrm{H}), 1.73-1.66(\mathrm{~m}, 2 \mathrm{H})$.

${ }^{13}$ C NMR (125 MHz, $\left.\mathbf{C D C l}_{3}\right) \delta 143.67,135.11,130.42,126.37,126.00,125.51,68.69$, $37.37,33.21,19.36$.

Spectroscopic data matches with previously reported data. ${ }^{8}$ 


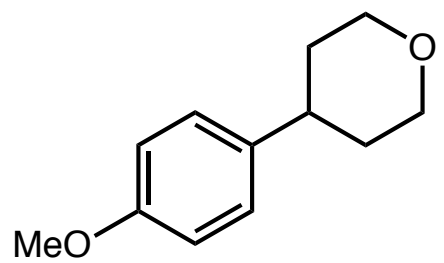

\section{4-(4-methoxyphenyl)tetrahydro-2H-pyran (4)}

Prepared following the general procedure outlined above using $\operatorname{Ir}\left[\mathrm{dF}\left(\mathrm{CF}_{3}\right) \text { ppy }\right]_{2}$ (dtbbpy)PF 6 (5.6 mg, $0.5 \mu \mathrm{mol}, 0.01$ equiv.), $\mathrm{NiCl}_{2} \bullet$ glyme (0.5 mg, $2.5 \mu \mathrm{mol}, 0.005$ equiv.), 4,4'-di-tert-butyl-2,2'-bipyridine ( $0.8 \mathrm{mg}, 3.0 \mu \mathrm{mol}, 0.006$ equiv, $\mathrm{Na}_{2} \mathrm{CO}_{3}$ (106.0 mg, 1.00 mmol, 2.0 equiv.), TTMSS (154 $\mu \mathrm{L}, 0.50 \mathrm{mmol}, 1.0$ equiv.), 4bromotetrahydro-2H-pyran ( $84 \mu \mathrm{L}, 0.75 \mathrm{mmol}, 1.5$ equiv.), 4-bromoanisole (63 $\mu \mathrm{L}, 94.0$ mg, $0.50 \mathrm{mmol}, 1.0$ equiv.), and DME (5.0 mL). Purification by column chromatography yielded the pure product as a clear oil (73 $\mathrm{mg}, 0.38 \mathrm{mmol}, 77 \%$ yield).

${ }^{1}$ H NMR (500 MHz, $\left.\mathbf{C D C l}_{3}\right) \delta 7.15(\mathrm{~d}, J=8.6 \mathrm{~Hz}, 2 \mathrm{H}), 6.87(\mathrm{~d}, J=8.6 \mathrm{~Hz}, 2 \mathrm{H}), 4.12$ $4.03(\mathrm{~m}, 2 \mathrm{H}), 3.80(\mathrm{~s}, 3 \mathrm{H}), 3.52(\mathrm{td}, J=11.4,2.8 \mathrm{~Hz}, 2 \mathrm{H}), 2.70(\mathrm{tt}, J=11.2,4.6 \mathrm{~Hz}, 1 \mathrm{H})$, $1.84-1.70(\mathrm{~m}, 4 \mathrm{H})$.

${ }^{13} \mathbf{C}$ NMR (125 MHz, $\left.\mathbf{C D C l}_{3}\right) \delta 158.0,138.1,127.6,113.9,68.5,55.3,40.7,34.2$.

Spectroscopic data matches with previously reported data. ${ }^{8}$ 


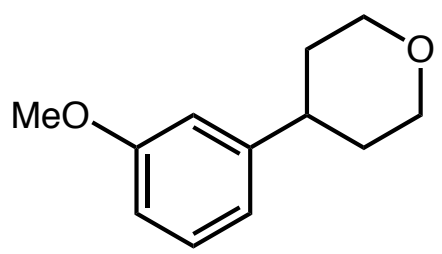

\section{4-(3-methoxyphenyl)tetrahydro-2H-pyran (6)}

Prepared following the general procedure outlined above using $\operatorname{Ir}\left[\mathrm{dF}_{(}\left(\mathrm{CF}_{3}\right) \text { ppy }\right]_{2}$ (dtbbpy)PF 6 (5.6 mg, $0.5 \mu \mathrm{mol}, 0.01$ equiv.), $\mathrm{NiCl}_{2} \bullet$ glyme (0.5 mg, $2.5 \mu \mathrm{mol}, 0.005$ equiv.), 4,4'-di-tert-butyl-2,2'-bipyridine ( $0.8 \mathrm{mg}, 3.0 \mu \mathrm{mol}, 0.006$ equiv, $\mathrm{Na}_{2} \mathrm{CO}_{3}$ (106.0 mg, 1.00 mmol, 2.0 equiv.), TTMSS (154 $\mu \mathrm{L}, 0.50$ mmol, 1.0 equiv.), 4bromotetrahydro-2H-pyran ( $84 \mu \mathrm{L}, 0.75$ mmol, 1.5 equiv.), 3-bromoanisole ( $63 \mu \mathrm{L}, 94.0$ $\mathrm{mg}, 0.50 \mathrm{mmol}, 1.0$ equiv.), and DME (5.0 mL). Purification by column chromatography yielded the pure product as a clear oil ( $79 \mathrm{mg}, 0.41 \mathrm{mmol}, 82 \%$ yield).

${ }^{1}$ H NMR (500 MHz, CDCl $) \delta 7.28(\mathrm{t}, J=7.7 \mathrm{~Hz}, 1 \mathrm{H}), 6.86(\mathrm{dt}, J=7.7,1.2 \mathrm{~Hz}, 1 \mathrm{H})$, $6.83-6.77(\mathrm{~m}, 2 \mathrm{H}), 4.14-4.08(\mathrm{~m}, 2 \mathrm{H}), 3.84(\mathrm{~s}, 3 \mathrm{H}), 3.56(\mathrm{td}, J=11.6,2.6 \mathrm{~Hz}, 2 \mathrm{H})$, $2.77(\mathrm{tt}, J=11.6,4.3 \mathrm{~Hz}, 1 \mathrm{H}), 1.91-1.77(\mathrm{~m}, 4 \mathrm{H})$.

${ }^{13}$ C NMR (125 MHz, CDCl $)$ ) $\delta 159.7,147.6,129.5,119.1,112.8,111.2,68.4,55.2$, 41.6, 33.9 .

Spectroscopic data matches with previously reported data. ${ }^{8}$ 


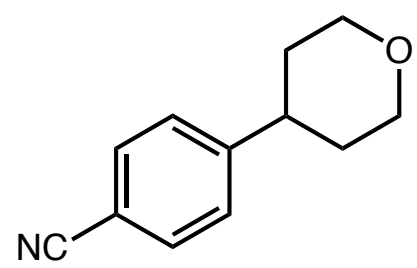

\section{4-(tetrahydro-2H-pyran-4-yl)benzonitrile (7)}

Prepared following the general procedure outlined above using $\operatorname{Ir}\left[\mathrm{dF}\left(\mathrm{CF}_{3}\right) \text { ppy }\right]_{2}$ (dtbbpy)PF 6 (5.6 mg, $0.5 \mu \mathrm{mol}, 0.01$ equiv.), $\mathrm{NiCl}_{2} \bullet$ glyme (0.5 mg, $2.5 \mu \mathrm{mol}, 0.005$ equiv.), 4,4'-di-tert-butyl-2,2'-bipyridine ( $0.8 \mathrm{mg}, 3.0 \mu \mathrm{mol}, 0.006$ equiv, $\mathrm{Na}_{2} \mathrm{CO}_{3}$ (106.0 mg, 1.00 mmol, 2.0 equiv.), TTMSS (154 $\mu \mathrm{L}, 0.50 \mathrm{mmol}, 1.0$ equiv.), 4bromotetrahydro-2H-pyran ( $84 \mu \mathrm{L}, 0.75 \mathrm{mmol}, 1.5$ equiv.), 4-bromobenzonitrile (91 mg, $0.50 \mathrm{mmol}, 1.0$ equiv.), and DME (5.0 mL). Purification by column chromatography yielded the pure product as a white solid (68 $\mathrm{mg}, 0.365 \mathrm{mmol}, 73 \%$ yield).

${ }^{1}$ H NMR (500 MHz, Acetone-d6) $\delta 7.72(\mathrm{~d}, J=8.3 \mathrm{~Hz}, 2 \mathrm{H}), 7.51(\mathrm{~d}, J=8.3 \mathrm{~Hz}, 2 \mathrm{H})$, $4.06-3.93(\mathrm{~m}, 2 \mathrm{H}), 3.49(\mathrm{ddd}, J=11.4,7.9,5.1 \mathrm{~Hz}, 2 \mathrm{H}), 3.01-2.88(\mathrm{~m}, 1 \mathrm{H}), 1.83-$ $1.69(\mathrm{~m}, 4 \mathrm{H})$.

${ }^{13}$ C NMR (126 MHz, Acetone-d 6 ) $\delta$ 152.70, 133.16, 128.80, 119.45, 110.82, 68.38, $42.37,34.23$.

IR (film) $v_{\max } 2942,2843,2226,1607,1386,1238,1123,1083,836.93 \mathrm{~cm}^{-1}$.

HRMS (ESI-TOF) m/z calcd. for $\mathrm{C}_{12} \mathrm{H}_{14} \mathrm{NO}\left([\mathrm{M}+\mathrm{H}]^{+}\right)$187.09971, found 187.0996. 


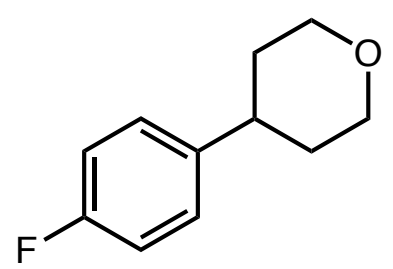

\section{4-(4-fluorophenyl)tetrahydro-2H-pyran (8)}

Prepared following the general procedure outlined above using $\operatorname{Ir}\left[\mathrm{dF}_{(\mathrm{CF}}\right)$ ppy $]_{2}$ (dtbbpy)PF 6 (5.6 mg, $0.5 \mu \mathrm{mol}, 0.01$ equiv.), $\mathrm{NiCl}_{2} \bullet$ glyme (0.5 mg, $2.5 \mu \mathrm{mol}, 0.005$ equiv.), 4,4'-di-tert-butyl-2,2'-bipyridine ( $0.8 \mathrm{mg}, 3.0 \mu \mathrm{mol}, 0.006$ equiv, $\mathrm{Na}_{2} \mathrm{CO}_{3}$ (106.0 mg, 1.00 mmol, 2.0 equiv.), TTMSS (154 $\mu \mathrm{L}, 0.50$ mmol, 1.0 equiv.), 4bromotetrahydro-2H-pyran ( $84 \mu \mathrm{L}, 0.75 \mathrm{mmol}, 1.5$ equiv.), 1-bromo-4fluorobenzene (55 $\mu \mathrm{L}, 87 \mathrm{mg}, 0.50 \mathrm{mmol}, 1.0$ equiv.), and $\mathrm{DME}(5.0 \mathrm{~mL})$. Purification by column chromatography yielded the pure product as a clear oil (66 mg, $0.36 \mathrm{mmol}, 73 \%$ yield).

${ }^{1}$ H NMR (500 MHz, CDCl$\left._{3}\right) \delta 7.21-7.15$ (m, 2H), $7.04-6.96$ (m, 2H), 4.12 - 4.03 (m, 2H), $3.52(\mathrm{td}, J=11.4,3.1 \mathrm{~Hz}, 2 \mathrm{H}), 2.74(\mathrm{ddd}, J=15.8,10.4,4.9 \mathrm{~Hz}, 1 \mathrm{H}), 1.84-1.70$ $(\mathrm{m}, 4 \mathrm{H})$.

${ }^{13}$ C NMR (125 MHz, CDCl $) \delta 161.38\left(\mathrm{~d}, J_{\mathrm{C}, \mathrm{F}}=244.0 \mathrm{~Hz}\right), 141.54\left(\mathrm{~d}, J_{\mathrm{C}, \mathrm{F}}=3.2 \mathrm{~Hz}\right)$, $128.07\left(\mathrm{~d}, J_{\mathrm{C}, \mathrm{F}}=7.8 \mathrm{~Hz}\right), 115.23\left(\mathrm{~d}, J_{\mathrm{C}, \mathrm{F}}=21.0 \mathrm{~Hz}\right), 68.35,40.87,34.11$.

${ }^{19}$ F NMR (282 MHz, $\left.\mathrm{CDCl}_{3}\right) \delta-117.06$.

IR (film) $v_{\max } 2939,2842,1603,1509,1221,1129,1016 \mathrm{~cm}^{-1}$.

HRMS (EI-TOF) calcd. for $\mathrm{C}_{11} \mathrm{H}_{13} \mathrm{FO}\left(\left[\mathrm{M}^{*}\right]^{+}\right)$180.0945, found 180.0952 . 


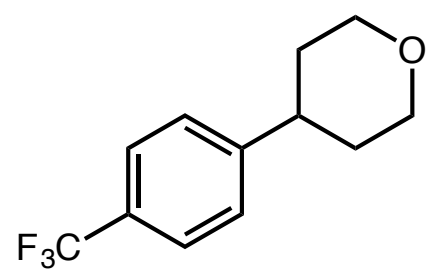

\section{4-(4-(trifluoromethyl)phenyl)tetrahydro-2H-pyran (9)}

Prepared following the general procedure outlined above using $\operatorname{Ir}\left[\mathrm{dF}\left(\mathrm{CF}_{3}\right) \text { ppy }\right]_{2}$ (dtbbpy)PF 6 (5.6 mg, $0.5 \mu \mathrm{mol}, 0.01$ equiv.), $\mathrm{NiCl}_{2} \bullet$ glyme (0.5 mg, $2.5 \mu \mathrm{mol}, 0.005$ equiv.), 4,4'-di-tert-butyl-2,2'-bipyridine ( $0.8 \mathrm{mg}, 3.0 \mu \mathrm{mol}, 0.006$ equiv, $\mathrm{Na}_{2} \mathrm{CO}_{3}(106.0$ mg, 1.00 mmol, 2.0 equiv.), TTMSS (154 $\mu \mathrm{L}, 0.50 \mathrm{mmol}, 1.0$ equiv.), 4bromotetrahydro-2H-pyran (84 $\mu \mathrm{L}, \quad 0.75$ mmol, 1.5 equiv.), 1-bromo-4(trifluoromethyl)benzene (70 $\mu \mathrm{L}, 113 \mathrm{mg}, 0.50 \mathrm{mmol}, 1.0$ equiv.), and DME $(5.0 \mathrm{~mL})$. Purification by column chromatography yielded the pure product as a clear oil $(89 \mathrm{mg}$, $0.39 \mathrm{mmol}, 78 \%$ yield).

${ }^{1}$ H NMR (500 MHz, $\left.\mathbf{C D C l}_{3}\right) \delta 7.57(\mathrm{~d}, J=7.9 \mathrm{~Hz}, 2 \mathrm{H}), 7.34(\mathrm{~d}, J=8.0 \mathrm{~Hz}, 2 \mathrm{H}), 4.16$ $4.04(\mathrm{~m}, 2 \mathrm{H}), 3.54(\mathrm{td}, J=11.6,2.5 \mathrm{~Hz}, 2 \mathrm{H}), 2.83(\mathrm{tt}, J=11.6,4.3 \mathrm{~Hz}, 1 \mathrm{H}), 1.92-1.72$ $(\mathrm{m}, 4 \mathrm{H})$.

${ }^{13}$ C NMR (125 MHz, CDCl 3 ) $\delta 149.8,128.7$ (q, $\left.J_{\mathrm{C}, \mathrm{F}}=31.5 \mathrm{~Hz}\right), 127.1,125.5\left(\mathrm{q}, J_{\mathrm{C}, \mathrm{F}}=\right.$ $3.8 \mathrm{~Hz}), 124.3\left(\mathrm{q}, J_{\mathrm{C}, \mathrm{F}}=272.2 \mathrm{~Hz}\right), 68.2,41.5,33.6$.

${ }^{19}$ F NMR (282 MHz, $\left.\mathbf{C D C l}_{3}\right) \delta-62.4$.

IR (film) $v_{\max } 2942,2844,1618,1323,1115,1067 \mathrm{~cm}^{-1}$.

HRMS (ESI-TOF) m/z calcd. for $\mathrm{C}_{12} \mathrm{H}_{14} \mathrm{~F}_{3} \mathrm{O}\left([\mathrm{M}+\mathrm{H}]^{+}\right)$231.0991, found 231.0993 . 


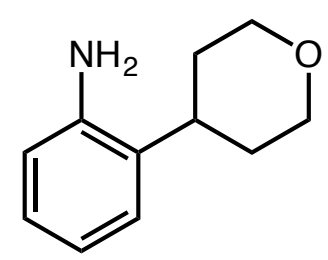

\section{2-(tetrahydro-2H-pyran-4-yl)aniline (10)}

Prepared following the general procedure outlined above using $\operatorname{Ir}\left[\mathrm{dF}\left(\mathrm{CF}_{3}\right) \text { ppy }\right]_{2}$ (dtbbpy)PF 6 (5.6 mg, $0.5 \mu \mathrm{mol}, 0.01$ equiv.), $\mathrm{NiCl}_{2} \bullet$ glyme (0.5 mg, $2.5 \mu \mathrm{mol}, 0.005$ equiv.), 4,4'-di-tert-butyl-2,2'-bipyridine ( $0.8 \mathrm{mg}, 3.0 \mu \mathrm{mol}, 0.006$ equiv, $\mathrm{Na}_{2} \mathrm{CO}_{3}(106.0$ mg, 1.00 mmol, 2.0 equiv.), TTMSS (154 $\mu \mathrm{L}, 0.50 \mathrm{mmol}, 1.0$ equiv.), 4bromotetrahydro-2H-pyran ( $84 \mu \mathrm{L}, 0.75 \mathrm{mmol}, 1.5$ equiv.), 2-bromoaniline ( $86 \mathrm{mg}, 0.50$ mmol, 1.0 equiv.), and DME (5.0 mL). The reaction was let run to 12 hours. Purification by column chromatography yielded the pure product as an orange oil $(58 \mathrm{mg}, 0.325$ mmol, $65 \%$ yield).

${ }^{1}$ H NMR (500 MHz, Acetone-d6) $\delta 7.04(\mathrm{dd}, J=7.8,1.6 \mathrm{~Hz}, 1 \mathrm{H}), 6.91(\mathrm{td}, J=7.6,1.6$ $\mathrm{Hz}, 1 \mathrm{H}), 6.69$ (dd, $J=8.0,1.4 \mathrm{~Hz}, 1 \mathrm{H}), 6.62(\mathrm{td}, J=7.4,1.4 \mathrm{~Hz}, 1 \mathrm{H}), 4.51(\mathrm{~s}, 2 \mathrm{H}), 3.96$ (ddd, $J=11.2,4.4,1.7 \mathrm{~Hz}, 2 \mathrm{H}), 3.51$ (td, $J=11.7,2.1 \mathrm{~Hz}, 2 \mathrm{H}), 2.90$ (tt, $J=11.7,3.7 \mathrm{~Hz}$, $1 \mathrm{H}), 1.80-1.72(\mathrm{~m}, 2 \mathrm{H}), 1.72-1.60(\mathrm{~m}, 2 \mathrm{H})$.

${ }^{13}$ C NMR (125 MHz, Acetone-d $) \delta$ 146.03, 130.08, 127.30, 126.39, 118.24, 116.19, $68.85,35.94,33.31$.

IR (film) $v_{\max } 3359,2940,2844,1622,1496,1121,1083 \mathrm{~cm}^{-1}$.

HRMS (ESI-TOF) m/z calcd. for $\mathrm{C}_{11} \mathrm{H}_{16} \mathrm{NO}\left([\mathrm{M}+\mathrm{H}]^{+}\right)$177.11536, found 177.11536. 


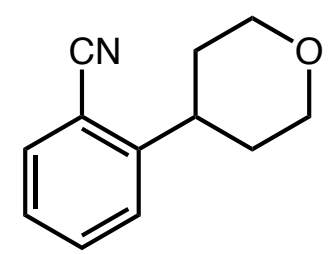

\section{2-(tetrahydro-2H-pyran-4-yl)benzonitrile (11)}

Prepared following the general procedure outlined above using $\operatorname{Ir}\left[\mathrm{dF}_{(\mathrm{CF}}\right)$ ppy $]_{2}$ (dtbbpy)PF 6 (5.6 mg, $0.5 \mu \mathrm{mol}, 0.01$ equiv.), $\mathrm{NiCl}_{2} \bullet$ glyme (0.5 mg, $2.5 \mu \mathrm{mol}, 0.005$ equiv.), 4,4'-di-tert-butyl-2,2'-bipyridine ( $0.8 \mathrm{mg}, 3.0 \mu \mathrm{mol}, 0.006$ equiv, $\mathrm{Na}_{2} \mathrm{CO}_{3}$ (106.0 mg, 1.00 mmol, 2.0 equiv.), TTMSS (154 $\mu \mathrm{L}, 0.50 \mathrm{mmol}, 1.0$ equiv.), 4bromotetrahydro-2H-pyran ( $84 \mu \mathrm{L}, 0.75 \mathrm{mmol}, 1.5$ equiv.), 2-bromobenzonitrile (91 $\mathrm{mg}$, $0.50 \mathrm{mmol}, 1.0$ equiv.), and DME $(5.0 \mathrm{~mL})$. The reaction was let run to 12 hours. Purification by column chromatography yielded the pure product as a white solid $(87 \mathrm{mg}$, $0.470 \mathrm{mmol}, 94 \%$ yield).

${ }^{1}$ H NMR (500 MHz, Acetone-d $) \delta 7.74(\mathrm{dd}, J=7.7,1.4 \mathrm{~Hz}, 1 \mathrm{H}), 7.69(\mathrm{td}, J=7.7,1.4$ $\mathrm{Hz}, 1 \mathrm{H}), 7.56(\mathrm{dd}, J=8.0,1.1 \mathrm{~Hz}, 1 \mathrm{H}), 7.43(\mathrm{td}, J=7.6,1.2 \mathrm{~Hz}, 1 \mathrm{H}), 4.02$ (ddt, $J=11.7$, 4.5, 1.1 Hz, 2H), $3.54(\mathrm{td}, J=11.7,2.4 \mathrm{~Hz}, 2 \mathrm{H}), 3.25-3.16(\mathrm{~m}, 1 \mathrm{H}), 1.89-1.73(\mathrm{~m}$, $4 \mathrm{H})$.

${ }^{13}$ C NMR (125 MHz, Acetone-d $\left.{ }_{6}\right) \delta$ 150.05, 134.25, 133.88, 127.93, 127.77, 118.38, $112.57,68.42,40.95,33.80$.

IR (film) $v_{\max } 2947,2844,2222,1238,1129,1089,760 \mathrm{~cm}^{-1}$.

HRMS (ESI-TOF) m/z calcd. for $\mathrm{C}_{12} \mathrm{H}_{14} \mathrm{NO}\left([\mathrm{M}+\mathrm{H}]^{+}\right)$187.09971, found 187.09939. 


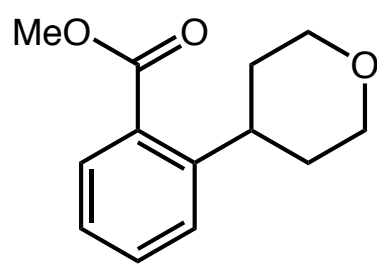

methyl 2-(tetrahydro-2H-pyran-4-yl)benzoate (12)

Prepared following the general procedure outlined above using $\operatorname{Ir}\left[\mathrm{dF}\left(\mathrm{CF}_{3}\right) \text { ppy }\right]_{2}$ (dtbbpy)PF 6 (5.6 mg, $0.5 \mu \mathrm{mol}, 0.01$ equiv.), $\mathrm{NiCl}_{2} \bullet$ glyme (0.5 mg, $2.5 \mu \mathrm{mol}, 0.005$ equiv.), 4,4'-di-tert-butyl-2,2'-bipyridine ( $0.8 \mathrm{mg}, 3.0 \mu \mathrm{mol}, 0.006$ equiv, $\mathrm{Na}_{2} \mathrm{CO}_{3}$ (106.0 mg, 1.00 mmol, 2.0 equiv.), TTMSS (154 $\mu \mathrm{L}, 0.50$ mmol, 1.0 equiv.), 4bromotetrahydro-2H-pyran ( $84 \mu \mathrm{L}, 0.75 \mathrm{mmol}, 1.5$ equiv.), methyl 2-bromobenzoate (70 $\mu \mathrm{L}, 108 \mathrm{mg}, 0.50 \mathrm{mmol}, 1.0$ equiv.), and DME (5.0 mL). The reaction was let run to 12 hours. Purification by column chromatography yielded the pure product as a white solid (87 mg, $0.470 \mathrm{mmol}, 94 \%$ yield).

${ }^{1}$ H NMR (500 MHz, $\left.\mathbf{C D C l}_{3}-d\right) \delta 7.80(\mathrm{dd}, J=7.7,1.5 \mathrm{~Hz}, 1 \mathrm{H}), 7.52-7.46(\mathrm{t}, J=7.5$, 1H), $7.41(\mathrm{~d}, J=7.5 \mathrm{~Hz}, 1 \mathrm{H}), 7.26(\mathrm{t}, J=8.0 \mathrm{~Hz}, 1 \mathrm{H}) . \delta 4.10-4.06(\mathrm{~m}, 2 \mathrm{H}), 3.65(\mathrm{tt}, J=$ 11.6, $4.0 \mathrm{~Hz}, 1 \mathrm{H}), 3.58$ (td, $J=11.6,2.4 \mathrm{~Hz}, 2 \mathrm{H}), 1.90-1.68(\mathrm{~m}, 5 \mathrm{H}), 4.10-4.04$ (m, $2 \mathrm{H})$.

${ }^{13}$ C NMR (125 MHz, CDCl $) \delta 168.58,146.93,132.20,130.40,129.80,127.09,126.06$, $68.69,52.23,37.57,34.04$.

IR (film) $v_{\max } 2950,2841,1718,1241,1085,754 \mathrm{~cm}^{-1}$.

HRMS (ESI-TOF) m/z calcd. for $\mathrm{C}_{13} \mathrm{H}_{17} \mathrm{O}_{3}\left([\mathrm{M}+\mathrm{H}]^{+}\right)$220.1099, found 220.1102. 


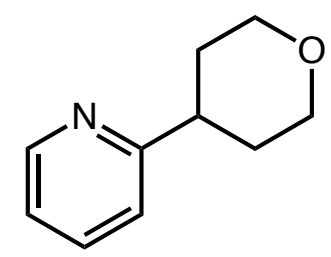

\section{2-(tetrahydro-2H-pyran-4-yl)pyridine (13)}

Prepared following the general procedure outlined above using $\operatorname{Ir}\left[\mathrm{dF}\left(\mathrm{CF}_{3}\right) \text { ppy }\right]_{2}$ (dtbbpy)PF 6 (5.6 mg, $0.5 \mu \mathrm{mol}, 0.01$ equiv.), $\mathrm{NiCl}_{2} \bullet$ glyme (0.5 mg, $2.5 \mu \mathrm{mol}, 0.005$ equiv.), 4,4'-di-tert-butyl-2,2'-bipyridine $(0.8 \mathrm{mg}, 3.0 \mu \mathrm{mol}, 0.006$ equiv, $\mathrm{LiOH}(24.0$ mg, 1.00 mmol, 2.0 equiv.), TTMSS (154 $\mu \mathrm{L}, 0.50$ mmol, 1.0 equiv.), 4bromotetrahydro-2H-pyran ( $84 \mu \mathrm{L}, 0.75 \mathrm{mmol}, 1.5$ equiv.), 2-bromopyridine ( $48 \mu \mathrm{L}, 79$ $\mathrm{mg}, 0.50 \mathrm{mmol}, 1.0$ equiv.), and DME (5.0 mL). Purification by column chromatography yielded the pure product as a clear oil (41 $\mathrm{mg}, 0.25 \mathrm{mmol}, 50 \%$ yield).

${ }^{1}$ H NMR (500 MHz, Acetone- $\left.\boldsymbol{d}_{\mathbf{6}}\right) \delta 8.50(\mathrm{ddd}, J=4.8,1.9,1.0 \mathrm{~Hz}, 1 \mathrm{H}), 7.69(\mathrm{td}, J=7.7$, $1.9 \mathrm{~Hz}, 1 \mathrm{H}), 7.29-7.24$ (m, 1H), 7.17 (ddd, $J=7.5,4.8,1.1 \mathrm{~Hz}, 1 \mathrm{H}), 4.00-3.95$ (m, 2H), $3.48(\mathrm{td}, J=11.7,2.4 \mathrm{~Hz}, 2 \mathrm{H}), 2.93(\mathrm{tt}, J=11.6,4.1 \mathrm{~Hz}, 1 \mathrm{H}), 1.91-1.73(\mathrm{~m}, 4 \mathrm{H})$.

${ }^{13}$ C NMR (125 MHz, Acetone- $\left.\boldsymbol{d}_{\mathbf{6}}\right) \delta$ 165.43, 150.01, 137.20, 122.18, 121.94, 68.37, 44.06, 33.23.

IR (film) $v_{\max } 2943,2842,1589,1472,1433,1237,1127,1087,748 \mathrm{~cm}^{-1}$.

HRMS (EI-TOF) m/z calcd. for $\mathrm{C}_{10} \mathrm{H}_{14} \mathrm{NO}\left([\mathrm{M}+\mathrm{H}]^{+}\right)$163.0997, found 163.0996. 


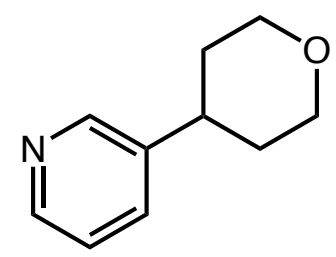

\section{3-(tetrahydro-2H-pyran-4-yl)pyridine (14)}

Prepared following the general procedure outlined above using $\operatorname{Ir}\left[\mathrm{dF}\left(\mathrm{CF}_{3}\right) \text { ppy }\right]_{2}$ (dtbbpy)PF 6 (5.6 mg, $0.5 \mu \mathrm{mol}, 0.01$ equiv.), $\mathrm{NiCl}_{2} \bullet$ glyme (0.5 mg, $2.5 \mu \mathrm{mol}, 0.005$ equiv.), 4,4'-di-tert-butyl-2,2'-bipyridine ( $0.8 \mathrm{mg}, 3.0 \mu \mathrm{mol}, 0.006$ equiv, $\mathrm{LiOH}(24.0$ mg, 1.00 mmol, 2.0 equiv.), TTMSS (154 $\mu \mathrm{L}, 0.50$ mmol, 1.0 equiv.), 4bromotetrahydro-2H-pyran ( $84 \mu \mathrm{L}, 0.75 \mathrm{mmol}, 1.5$ equiv.), 3-bromopyridine ( $48 \mu \mathrm{L}, 79$ $\mathrm{mg}, 0.50 \mathrm{mmol}, 1.0$ equiv.), and DME (5.0 mL). Purification by column chromatography yielded the pure product as a clear oil ( $64 \mathrm{mg}, 0.4 \mathrm{mmol}, 80 \%$ yield).

${ }^{1}$ H NMR (500 MHz, Acetone- $\left.\boldsymbol{d}_{\mathbf{6}}\right) \boldsymbol{\delta} 8.51(\mathrm{~s}, 1 \mathrm{H}), 8.45-8.41(\mathrm{~m}, 1 \mathrm{H}), 7.66(\mathrm{dt}, J=8.1$, $1.9 \mathrm{~Hz}, 1 \mathrm{H}), 7.30$ (dd, $J=7.9,4.7 \mathrm{~Hz}, 1 \mathrm{H}), 4.05-3.94(\mathrm{~m}, 2 \mathrm{H}), 3.53-3.45$ (m, 2H), $2.85(\mathrm{ddd}, J=15.9,10.6,6.2 \mathrm{~Hz}, 1 \mathrm{H}), 1.75(\mathrm{td}, J=9.7,8.4,3.7 \mathrm{~Hz}, 4 \mathrm{H})$.

${ }^{13}$ C NMR (125 MHz, Acetone- $\left.\boldsymbol{d}_{\mathbf{6}}\right) \delta$ 149.69, 148.54, 142.07, 134.62, 124.24, 68.45, 39.76, 34.38.

IR (film) $v_{\max } 3414,2940,2843,1575,1425,1238,1126,1085,1019,839 \mathrm{~cm}^{-1}$.

HRMS (EI-TOF) m/z calcd. for $\mathrm{C}_{10} \mathrm{H}_{14} \mathrm{NO}\left([\mathrm{M}+\mathrm{H}]^{+}\right)$163.0997, found 163.0995. 


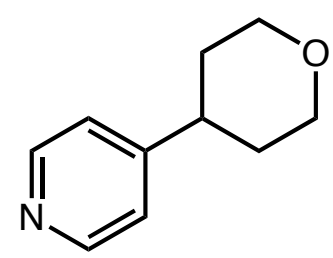

\section{4-(tetrahydro-2H-pyran-4-yl)pyridine (15)}

Prepared following the general procedure outlined above using $\operatorname{Ir}\left[\mathrm{dF}\left(\mathrm{CF}_{3}\right) \text { ppy }\right]_{2}$ (dtbbpy)PF 6 (5.6 mg, $10.0 \mu \mathrm{mol}, 0.01$ equiv.), $\mathrm{NiCl}_{2} \bullet$ glyme (0.6 mg, $5.0 \mu \mathrm{mol}, 0.005$ equiv.), 4,4'-di-tert-butyl-2,2'-bipyridine ( $0.8 \mathrm{mg}, 12.0 \mu \mathrm{mol}, 0.006$ equiv.), $\mathrm{LiOH}$ (24.0 mg, 2.0 mmol, 2.0 equiv.), TTMSS (154 $\mu \mathrm{L}, 1.00 \mathrm{mmol}, 1.0$ equiv.), 4-bromotetrahydro2H-pyran (84 $\mu \mathrm{L}, 0.75 \mathrm{mmol}, 1.5$ equiv.), 4-bromopyridine $\bullet$ hydrochloride (97 $\mathrm{mg}, 0.50$ mmol, 1.0 equiv.), and DME (5.0 mL). Purification by column chromatography yielded the pure product as a white crystalline solid (66 $\mathrm{mg}, 0.40 \mathrm{mmol}, 81 \%$ yield).

${ }^{1}$ H NMR (500 MHz, $\left.\mathbf{C D C l}_{3}\right) \delta 8.53(\mathrm{~d}, J=5.2 \mathrm{~Hz}, 2 \mathrm{H}), 7.14(\mathrm{~d}, J=6.1 \mathrm{~Hz}, 2 \mathrm{H}), 4.13-$ $4.07(\mathrm{~m}, 2 \mathrm{H}), 3.58-3.49(\mathrm{~m}, 2 \mathrm{H}), 2.76(\mathrm{tt}, J=10.8,5.2 \mathrm{~Hz}, 1 \mathrm{H}), 1.86-1.74(\mathrm{~m}, 4 \mathrm{H})$.

${ }^{13} \mathbf{C}$ NMR (125 MHz, $\left.\mathbf{C D C l}_{3}\right) \delta 154.3,150.0,122.2,68.0,40.8,32.9$.

IR (film) $v_{\max } 3413,3025,2941,2843,1598,1127 \mathrm{~cm}^{-1}$.

HRMS (ESI-TOF) m/z calcd. for $\mathrm{C}_{10} \mathrm{H}_{14} \mathrm{NO}\left([\mathrm{M}+\mathrm{H}]^{+}\right)$164.1070, found 164.1070. 


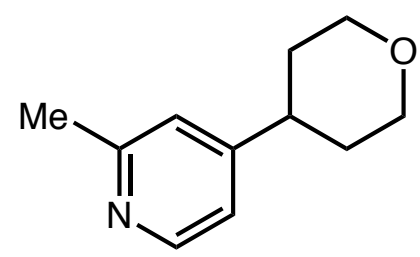

2-methyl-4-(tetrahydro-2H-pyran-4-yl)pyridine (16)

Prepared following the general procedure outlined above using $\operatorname{Ir}\left[\mathrm{dF}_{(}\left(\mathrm{CF}_{3}\right) \text { ppy }\right]_{2}$ (dtbbpy)PF 6 (5.6 mg, $5.0 \mu \mathrm{mol}, 0.01$ equiv.), $\mathrm{NiCl}_{2} \bullet$ glyme (0.6 mg, $2.5 \mu \mathrm{mol}, 0.005$ equiv.), 4,4'-di-tert-butyl-2,2'-bipyridine ( $0.8 \mathrm{mg}, 3.0 \mu \mathrm{mol}, 0.006$ equiv.), quinuclidine (0.6 mg, $10.0 \mu \mathrm{mol}, 0.01$ equiv.), LiOH (24.0 mg, $1.0 \mathrm{mmol}, 2.0$ equiv.), TTMSS (154 $\mu \mathrm{L}, 1.00$ mmol, 1.0 equiv.), 4-bromotetrahydro-2H-pyran ( $84 \mu \mathrm{L}, 0.75 \mathrm{mmol}, 1.5$ equiv.), 4-bromo-2-methylpyridine (86 mg, $0.50 \mathrm{mmol}, 1.0$ equiv.), and DME (5.0 mL). Purification by column chromatography yielded the pure product as a clear oil (64 mg, $0.36 \mathrm{mmol}, 72 \%$ yield).

${ }^{1}$ H NMR (500 MHz, CDCl 3 ) $\delta 8.41(\mathrm{~d}, J=5.2 \mathrm{~Hz}, 1 \mathrm{H}), 7.00(\mathrm{~d}, J=1.6 \mathrm{~Hz}, 1 \mathrm{H}), 6.95$ $(\mathrm{dd}, J=5.3,1.7 \mathrm{~Hz}, 1 \mathrm{H}), 4.08(\mathrm{ddd}, J=11.4,4.5,2.0 \mathrm{~Hz}, 2 \mathrm{H}), 3.52(\mathrm{td}, J=11.4,3.0 \mathrm{~Hz}$, $2 \mathrm{H}), 2.78-2.66(\mathrm{~m}, 1 \mathrm{H}), 2.54(\mathrm{~s}, 3 \mathrm{H}), 1.84-1.73(\mathrm{~m}, 4 \mathrm{H})$.

${ }^{13}$ C NMR (125 MHz, CDCl 3 ) $\delta$ 158.54, 154.66, 149.28, 121.68, 119.29, 68.06, 40.85, $32.97,24.48$.

IR (film) $v_{\max } 3400,2936,2843,1604,1558,1128,1086 \mathrm{~cm}^{-1}$.

HRMS (ESI-TOF) m/z calcd. for $\mathrm{C}_{11} \mathrm{H}_{16} \mathrm{NO}\left([\mathrm{M}+\mathrm{H}]^{+}\right)$177.1154, found 177.1156. 


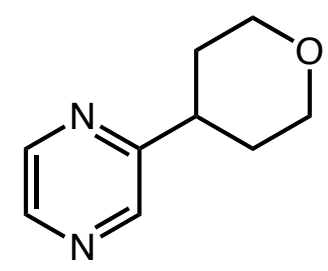

\section{2-(tetrahydro-2H-pyran-4-yl)pyrazine (17)}

Prepared following the general procedure outlined above using $\operatorname{Ir}\left[\mathrm{dF}_{(}\left(\mathrm{CF}_{3}\right) \text { ppy }\right]_{2}$ (dtbbpy)PF 6 (5.6 mg, $10.0 \mu \mathrm{mol}, 0.01$ equiv.), $\mathrm{NiCl}_{2} \bullet$ glyme (0.6 mg, $5.0 \mu \mathrm{mol}, 0.005$ equiv.), 4,4'-di-tert-butyl-2,2'-bipyridine ( $0.8 \mathrm{mg}, 12.0 \mu \mathrm{mol}, 0.006$ equiv.), $\mathrm{LiOH}(24.0$ mg, 2.0 mmol, 2.0 equiv.), TTMSS (154 $\mu \mathrm{L}, 1.00 \mathrm{mmol}, 1.0$ equiv.), 4-bromotetrahydro2H-pyran ( $84 \mu \mathrm{L}, 0.75 \mathrm{mmol}, 1.5$ equiv.), 2-bromopyrazine ( $45 \mu \mathrm{L}, 79 \mathrm{mg}, 0.50 \mathrm{mmol}$, 1.0 equiv.), and DME $(5.0 \mathrm{~mL})$. Purification by column chromatography yielded the pure product as a clear oil ( $41 \mathrm{mg}, 0.25 \mathrm{mmol}, 50 \%$ yield).

${ }^{1}$ H NMR (500 MHz, Acetone- $\left.d_{6}\right) \delta 8.56(\mathrm{~d}, J=1.6 \mathrm{~Hz}, 1 \mathrm{H}), 8.53(\mathrm{dd}, J=2.5,1.5 \mathrm{~Hz}$, 1H), 8.45 (d, $J=2.5 \mathrm{~Hz}, 1 \mathrm{H}), 4.04-3.96$ (m, 2H), 3.51 (td, $J=11.7,2.3 \mathrm{~Hz}, 2 \mathrm{H}), 3.06$ (tt, $J=11.7,4.1 \mathrm{~Hz}, 1 \mathrm{H}), 1.88(\mathrm{dtd}, J=13.2,11.9,4.4 \mathrm{~Hz}, 2 \mathrm{H}), 1.83-1.77(\mathrm{~m}, 2 \mathrm{H})$.

${ }^{13}$ C NMR (125 MHz, Acetone- $\left.d_{6}\right) \delta$ 160.67, 144.93, 144.49, 143.60, 68.16, 41.48, 32.69.

IR (film) $v_{\max } 2950,2844,1408,1239,1150,1086,1022,845 \mathrm{~cm}^{-1}$.

HRMS (ESI-TOF) $\mathrm{m} / \mathrm{z}$ calcd. for $\mathrm{C}_{9} \mathrm{H}_{13} \mathrm{~N}_{2} \mathrm{O}\left([\mathrm{M}+\mathrm{H}]^{+}\right)$164.09496, found 164.0952. 


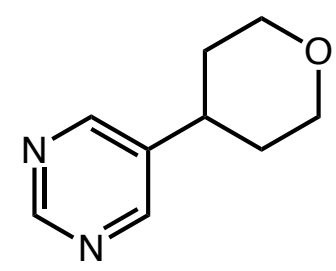

\section{5-(tetrahydro-2H-pyran-4-yl)pyrimidine (18)}

Prepared following the general procedure outlined above using $\operatorname{Ir}\left[\mathrm{dF}\left(\mathrm{CF}_{3}\right) \text { ppy }\right]_{2}$ (dtbbpy)PF 6 (5.6 mg, $10.0 \mu \mathrm{mol}, 0.01$ equiv.), $\mathrm{NiCl}_{2} \bullet$ glyme (0.6 mg, $5.0 \mu \mathrm{mol}, 0.005$ equiv.), 4,4'-di-tert-butyl-2,2'-bipyridine ( $0.8 \mathrm{mg}, 12.0 \mu \mathrm{mol}, 0.006$ equiv.), $\mathrm{LiOH}$ (24.0 mg, 2.0 mmol, 2.0 equiv.), TTMSS (154 $\mu \mathrm{L}, 1.00$ mmol, 1.0 equiv.), 4-bromotetrahydro2H-pyran (84 $\mu \mathrm{L}, 0.75 \mathrm{mmol}, 1.5$ equiv.), 5-chloropyrimidine (57.3 mg, $0.50 \mathrm{mmol}, 1.0$ equiv.), and DME (5.0 mL). Purification by column chromatography yielded the pure product as a white crystalline solid (53 $\mathrm{mg}, 0.32 \mathrm{mmol}, 64 \%$ yield).

${ }^{1}$ H NMR (500 MHz, CDCl $) \delta 9.10$ (s, 1H), 8.62 (s, 2H), 4.11 (ddd, $J=11.5,4.2,1.8$ $\mathrm{Hz}, 2 \mathrm{H}), 3.54(\mathrm{td}, J=11.5,2.8 \mathrm{~Hz}, 2 \mathrm{H}), 2.81(\mathrm{tt}, J=11.5,4.6 \mathrm{~Hz}, 1 \mathrm{H}), 1.92-1.76(\mathrm{~m}$, $5 \mathrm{H})$.

${ }^{13}$ C NMR (125 MHz, $\left.\mathbf{C D C l}_{3}\right) \delta 157.2,155.6,138.2,67.9,37.1,33.0$.

IR (film) $v_{\max } 3402,2963,2849,1562,1446,1408,1387,1276,1270,1234,1163,1126$, $1083,1016 \mathrm{~cm}^{-1}$.

HRMS (ESI-TOF) m/z calcd. for $\mathrm{C}_{9} \mathrm{H}_{13} \mathrm{~N}_{2} \mathrm{O}\left([\mathrm{M}+\mathrm{H}]^{+}\right)$165.1022, found 165.1022. 


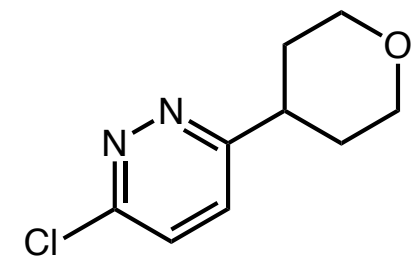

3-chloro-6-(tetrahydro-2H-pyran-4-yl)pyridazine (19)

Prepared following the general procedure outlined above using $\operatorname{Ir}\left[\mathrm{dF}_{(}\left(\mathrm{CF}_{3}\right) \text { ppy }\right]_{2}$ (dtbbpy) $\mathrm{PF}_{6}\left(5.6 \mathrm{mg}, 10.0 \mu \mathrm{mol}, 0.01\right.$ equiv.), $\mathrm{NiCl}_{2} \bullet$ glyme (11 mg, 0.05 mmol, 0.1 equiv.), 4,4'-di-tert-butyl-2,2'-bipyridine ( $15 \mathrm{mg}, 0.055 \mathrm{mmol}, 0.11$ equiv.), $\mathrm{LiOH}$ (24.0 mg, 2.0 mmol, 2.0 equiv.), TTMSS (154 $\mu \mathrm{L}, 1.00 \mathrm{mmol}, 1.0$ equiv.), 4-bromotetrahydro2H-pyran ( $84 \mu \mathrm{L}, 0.75 \mathrm{mmol}, 1.5$ equiv.), 3,6-dichloropyrdazine (74 mg, $0.50 \mathrm{mmol}, 1.0$ equiv.), and DME $(5.0 \mathrm{~mL})$. Purification by column chromatography yielded the pure product as a white solid (53 $\mathrm{mg}, 0.265 \mathrm{mmol}, 53 \%$ yield).

${ }^{1} \mathbf{H}$ NMR (500 MHz, CDCl $) \delta 7.47(\mathrm{~d}, J=8.8 \mathrm{~Hz}, 1 \mathrm{H}), 7.34(\mathrm{~d}, J=8.8 \mathrm{~Hz}, 1 \mathrm{H}), 4.12$ (dt, $J=11.6,3.3 \mathrm{~Hz}, 2 \mathrm{H}), 3.62-3.54(\mathrm{~m}, 2 \mathrm{H}), 3.30-3.18(\mathrm{~m}, 1 \mathrm{H}), 1.96-1.88(\mathrm{~m}, 4 \mathrm{H})$.

${ }^{13}$ C NMR (125 MHz, CDCl $)$ ) $\delta$ 165.13, 155.53, 128.63, 127.06, 67.89, 41.39, 32.13.

IR (film) $v_{\max } 3036,2957,2856,1418,1234,1144,1125,1085,979,871 \mathrm{~cm}^{-1}$.

HRMS (ESI-TOF) $\mathrm{m} / \mathrm{z}$ calcd. for $\mathrm{C}_{9} \mathrm{H}_{12} \mathrm{ClN}_{2} \mathrm{O}\left([\mathrm{M}+\mathrm{H}]^{+}\right)$198.0559, found 198.0561. 


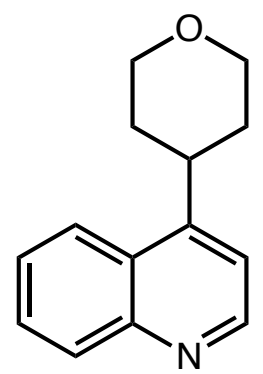

\section{4-(tetrahydro-2H-pyran-4-yl)quinoline (20)}

Prepared following the general procedure outlined above using $\operatorname{Ir}\left[\mathrm{dF}_{(}\left(\mathrm{CF}_{3}\right) \text { ppy }\right]_{2}$ (dtbbpy)PF 6 (5.6 mg, $10.0 \mu \mathrm{mol}, 0.01$ equiv.), $\mathrm{NiCl}_{2} \bullet$ glyme (5.5 mg, $0.025 \mathrm{mmol}, 0.05$ equiv.), 4,4'-di-tert-butyl-2,2'-bipyridine ( $8 \mathrm{mg}, 0.030 \mathrm{mmol}, 0.06$ equiv.), LiOH (24.0 mg, 2.0 mmol, 2.0 equiv.), TTMSS (154 $\mu \mathrm{L}, 1.00 \mathrm{mmol}, 1.0$ equiv.), 4-bromotetrahydro2H-pyran ( $84 \mu \mathrm{L}, 0.75 \mathrm{mmol}, 1.5$ equiv.), 4-bromoquinoline (104 mg, $0.50 \mathrm{mmol}, 1.0$ equiv.), and DME (5.0 mL). Purification by column chromatography yielded the pure product as a white solid ( $80 \mathrm{mg}, 0.375 \mathrm{mmol}, 75 \%$ yield).

${ }^{1}$ H NMR (300 MHz, Acetone- $\left.d_{6}\right) \delta 8.87(\mathrm{~d}, J=4.6 \mathrm{~Hz}, 1 \mathrm{H}), 8.34-8.27(\mathrm{~m}, 1 \mathrm{H}), 8.12-$ $8.03(\mathrm{~m}, 1 \mathrm{H}), 7.75$ (ddd, $J=8.5,6.8,1.5 \mathrm{~Hz}, 1 \mathrm{H}), 7.63$ (ddd, $J=8.4,6.8,1.4 \mathrm{~Hz}, 1 \mathrm{H}$ ), $7.44(\mathrm{~d}, J=4.6 \mathrm{~Hz}, 1 \mathrm{H}), 4.07$ (dt, $J=11.3,3.0 \mathrm{~Hz}, 2 \mathrm{H}), 3.84-3.63(\mathrm{~m}, 3 \mathrm{H}), 1.90(\mathrm{tt}, J=$ $5.9,2.9 \mathrm{~Hz}, 4 \mathrm{H})$.

${ }^{13}$ C NMR (125 MHz, Acetone- $\left.d_{6}\right) \delta$ 152.11, 151.36, 149.60, 131.30, 129.61, 127.48, $127.15,124.03,118.57,68.48,36.74,33.98$.

IR (film) $v_{\max } 2952,2848,1591,1509,1264,1127,1086,898 \mathrm{~cm}^{-1}$.

HRMS (ESI-TOF) $\mathrm{m} / \mathrm{z}$ calcd. for $\mathrm{C}_{14} \mathrm{H}_{16} \mathrm{NO}\left([\mathrm{M}+\mathrm{H}]^{+}\right)$213.1153, found 213.1154 . 


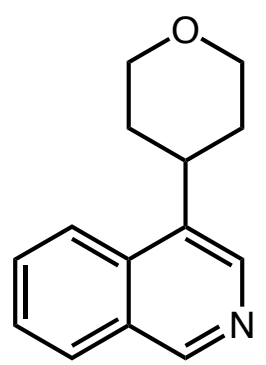

\section{4-(tetrahydro-2H-pyran-4-yl)isoquinoline (21)}

Prepared following the general procedure outlined above using $\operatorname{Ir}\left[\mathrm{dF}_{(\mathrm{CF}}\right)$ ppy $]_{2}$ (dtbbpy)PF 6 (5.6 mg, $10.0 \mu \mathrm{mol}, 0.01$ equiv.), $\mathrm{NiCl}_{2} \bullet$ glyme (5.5 mg, $0.025 \mathrm{mmol}, 0.05$ equiv.), 4,4'-di-tert-butyl-2,2'-bipyridine ( $8 \mathrm{mg}, 0.030 \mathrm{mmol}, 0.06$ equiv.), $\mathrm{LiOH}$ (24.0 mg, 2.0 mmol, 2.0 equiv.), TTMSS (154 $\mu \mathrm{L}, 1.00 \mathrm{mmol}, 1.0$ equiv.), 4-bromotetrahydro2H-pyran ( $84 \mu \mathrm{L}, 0.75 \mathrm{mmol}, 1.5$ equiv.), 4-bromoisoquinoline (104 mg, $0.50 \mathrm{mmol}, 1.0$ equiv.), and DME (5.0 mL). Purification by column chromatography yielded the pure product as a white solid ( $83 \mathrm{mg}, 0.390 \mathrm{mmol}, 78 \%$ yield).

${ }^{1}$ H NMR (300 MHz, Acetone-d $) \delta 9.17(\mathrm{~s}, 1 \mathrm{H}), 8.46(\mathrm{~s}, 1 \mathrm{H}), 8.25(\mathrm{~d}, J=8.6 \mathrm{~Hz}, 1 \mathrm{H})$, $8.12(\mathrm{~d}, J=8.1 \mathrm{~Hz}, 1 \mathrm{H}), 7.81(\mathrm{ddd}, J=8.5,6.7,1.5 \mathrm{~Hz}, 1 \mathrm{H}), 7.68(\mathrm{t}, J=7.5 \mathrm{~Hz}, 1 \mathrm{H})$, $4.10-4.01(\mathrm{~m}, 2 \mathrm{H}), 3.75-3.57(\mathrm{~m}, 3 \mathrm{H}), 2.00-1.85(\mathrm{~m}, 4 \mathrm{H})$.

${ }^{13}$ C NMR (125 MHz, Acetone-d $\left.d_{6}\right) \delta 152.05,141.00,135.23,134.55,131.12,129.38$, $129.26,127.70,123.11,68.72,35.83,34.23$.

IR (film) $v_{\max } 2950,2916,2842,1584,1383,1127,1085,904,855 \mathrm{~cm}^{-1}$.

HRMS (ESI-TOF) m/z calcd. for $\mathrm{C}_{14} \mathrm{H}_{16} \mathrm{NO}\left([\mathrm{M}+\mathrm{H}]^{+}\right)$213.1153, found 213.1156. 


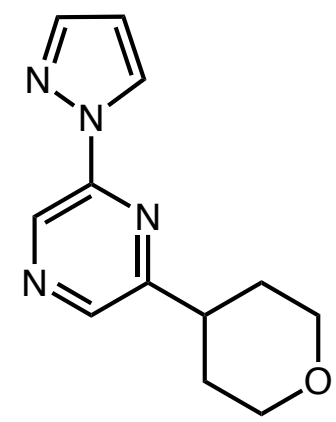

\section{2-(1H-pyrazol-1-yl)-6-(tetrahydro-2H-pyran-4-yl)pyrazine (22)}

Prepared following the general procedure outlined above using $\operatorname{Ir}\left[\mathrm{dF}\left(\mathrm{CF}_{3}\right) \text { ppy }\right]_{2}$ (dtbbpy)PF 6 (5.6 mg, $10.0 \mu \mathrm{mol}, 0.01$ equiv.), $\mathrm{NiCl}_{2} \bullet$ glyme (5.5 mg, $0.025 \mathrm{mmol}, 0.05$ equiv.), 4,4'-di-tert-butyl-2,2'-bipyridine ( $8 \mathrm{mg}, 0.030 \mathrm{mmol}, 0.06$ equiv.), $\mathrm{LiOH}$ (24.0 mg, 2.0 mmol, 2.0 equiv.), TTMSS (154 $\mu \mathrm{L}, 1.00 \mathrm{mmol}, 1.0$ equiv.), 4-bromotetrahydro2H-pyran ( $84 \mu \mathrm{L}, 0.75 \mathrm{mmol}, 1.5$ equiv.), 2-chloro-6-(1H-pyrazol-1-yl)pyrazine (90 mg, $0.50 \mathrm{mmol}, 1.0$ equiv.), and DME (5.0 mL). Purification by column chromatography yielded the pure product as a white solid $(99 \mathrm{mg}, 0.430 \mathrm{mmol}, 86 \%$ yield).

${ }^{1}$ H NMR (500 MHz, Acetone-d d $_{\text {) }} \delta 9.08(\mathrm{~s}, 1 \mathrm{H}), 8.68(\mathrm{~d}, J=2.6 \mathrm{~Hz}, 1 \mathrm{H}), 8.52(\mathrm{~s}, 1 \mathrm{H})$, $7.83(\mathrm{~d}, J=1.6 \mathrm{~Hz}, 1 \mathrm{H}), 6.60(\mathrm{dd}, J=2.6,1.6 \mathrm{~Hz}, 1 \mathrm{H}), 4.06-3.98(\mathrm{~m}, 2 \mathrm{H}), 3.54(\mathrm{td}, J=$ 11.7, $2.4 \mathrm{~Hz}, 2 \mathrm{H}), 3.14(\mathrm{tt}, J=11.7,4.1 \mathrm{~Hz}, 1 \mathrm{H}), 2.02-1.82(\mathrm{~m}, 4 \mathrm{H})$.

${ }^{13}$ C NMR (125 MHz, Acetone-d $)$ ) $158.49,147.58,143.68,141.41,133.25,128.13$, 109.24, 68.08, 40.98, 32.47.

IR (film) $v_{\max } 3161,3117,3076,2957,2844,1532,1454,1400,961 \mathrm{~cm}^{-1}$.

HRMS (ESI-TOF) m/z calcd. for $\mathrm{C}_{12} \mathrm{H}_{15} \mathrm{~N}_{4} \mathrm{O}\left([\mathrm{M}+\mathrm{H}]^{+}\right)$230.1167, found 230.1164. 


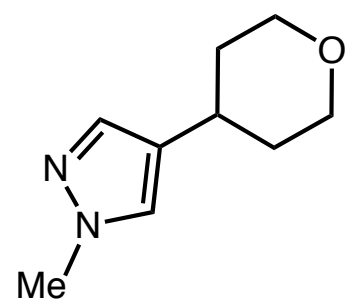

\section{1-methyl-4-(tetrahydro-2H-pyran-4-yl)-1H-pyrazole (23)}

Prepared following the general procedure outlined above using $\operatorname{Ir}\left[\mathrm{dF}\left(\mathrm{CF}_{3}\right) \text { ppy }\right]_{2}$ (dtbbpy)PF 6 (5.6 mg, $10.0 \mu \mathrm{mol}, 0.01$ equiv.), $\mathrm{NiCl}_{2} \bullet$ glyme (11 mg, $0.05 \mathrm{mmol}, 0.1$ equiv.), 4,4'-di-tert-butyl-2,2'-bipyridine (15 mg, $0.055 \mathrm{mmol}, 0.11$ equiv.), $\mathrm{LiOH}$ (24.0 mg, 2.0 mmol, 2.0 equiv.), TTMSS (154 $\mu \mathrm{L}, 1.00$ mmol, 1.0 equiv.), 4-bromotetrahydro2H-pyran ( $84 \mu \mathrm{L}, 0.75 \mathrm{mmol}, 1.5$ equiv.), 4-bromo-1-methyl-1H-pyrazole (52 $\mu \mathrm{L}, 80 \mathrm{mg}$, $0.50 \mathrm{mmol}, 1.0$ equiv.), and DME $(5.0 \mathrm{~mL})$. Purification by column chromatography $(10 \% \mathrm{MeOH}$ in $\mathrm{DCM})$ yielded the pure product as a white solid $(55 \mathrm{mg}, 0.330 \mathrm{mmol}$, $66 \%$ yield).

${ }^{1}$ H NMR (500 MHz, Acetone-d d $_{\text {) }} \delta 7.37$ (s, 1H), $7.26(\mathrm{~s}, 1 \mathrm{H}), 3.93-3.86(\mathrm{~m}, 2 \mathrm{H}), 3.80$ (s, 3H), $3.42(\mathrm{td}, J=11.8,2.1 \mathrm{~Hz}, 2 \mathrm{H}), 2.70(\mathrm{tt}, J=11.7,3.9 \mathrm{~Hz}, 1 \mathrm{H}), 1.78$ (ddd, $J=$ 13.2, 4.0, 2.0 Hz, 2H), 1.57 (dtd, $J=13.2,11.7,4.3 \mathrm{~Hz}, 2 \mathrm{H})$.

${ }^{13}$ C NMR (125 MHz, Acetone-d $\left.{ }_{6}\right) \delta$ 137.19, 127.61, 127.40, 68.28, 38.78, 35.23, 32.21.

IR (film) $v_{\max } 3437,2933,2844,1442,1387,1237,1088,987,826 \mathrm{~cm}^{-1}$.

HRMS (ESI-TOF) m/z calcd. for $\mathrm{C}_{9} \mathrm{H}_{15} \mathrm{~N}_{2} \mathrm{O}\left([\mathrm{M}+\mathrm{H}]^{+}\right)$166.1106, found 166.1103. 


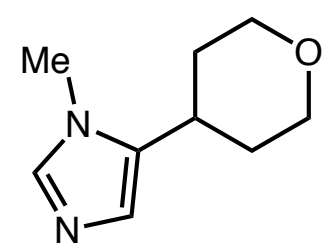

\section{1-methyl-5-(tetrahydro-2H-pyran-4-yl)-1H-imidazole (24)}

Prepared following the general procedure outlined above using $\operatorname{Ir}\left[\mathrm{dF}_{(}\left(\mathrm{CF}_{3}\right) \text { ppy }\right]_{2}$ (dtbbpy)PF 6 (5.6 mg, $10.0 \mu \mathrm{mol}, 0.01$ equiv.), $\mathrm{NiCl}_{2} \bullet$ glyme (11 mg, $0.05 \mathrm{mmol}, 0.1$ equiv.), 4,4'-di-tert-butyl-2,2'-bipyridine (15 mg, $0.055 \mathrm{mmol}, 0.11$ equiv.), $\mathrm{LiOH}$ (24.0 mg, 2.0 mmol, 2.0 equiv.), TTMSS (154 $\mu \mathrm{L}, 1.00 \mathrm{mmol}, 1.0$ equiv.), 4-bromotetrahydro2H-pyran ( $84 \mu \mathrm{L}, 0.75 \mathrm{mmol}, 1.5$ equiv.), 5-bromo-1-methyl-1H-imidazole (80 mg, 0.50 mmol, 1.0 equiv.), and DME (5.0 mL). Purification by column chromatography (10\% $\mathrm{MeOH}$ in DCM) yielded the pure product as a white solid (50 mg, $0.300 \mathrm{mmol}, 60 \%$ yield).

${ }^{1}$ H NMR (500 MHz, Acetone-d $\left.d_{6}\right) \delta 7.40(\mathrm{~s}, 1 \mathrm{H}), 6.72(\mathrm{~s}, 1 \mathrm{H}), 3.98-3.90(\mathrm{~m}, 2 \mathrm{H}), 3.64$ (s, 3H), $3.48(\mathrm{td}, J=11.8,2.0 \mathrm{~Hz}, 2 \mathrm{H}), 2.88$ (ddt, $J=11.8,8.0,3.8 \mathrm{~Hz}, 1 \mathrm{H}), 1.82$ (ddd, $J$ $=13.3,3.9,2.1 \mathrm{~Hz}, 2 \mathrm{H}), 1.62(\mathrm{dtd}, J=13.2,11.7,4.2 \mathrm{~Hz}, 2 \mathrm{H})$.

${ }^{13}$ C NMR (125 MHz, Acetone- $\left.\boldsymbol{d}_{6}\right) \delta$ 138.88, 137.24, 125.48, 68.18, 33.52, 31.89, 31.40.

IR (film) $v_{\max } 3365,2945,2846,1653,1503,1240,1124,1085,826,665 \mathrm{~cm}^{-1}$.

HRMS (ESI-TOF) m/z calcd. for $\mathrm{C}_{9} \mathrm{H}_{15} \mathrm{~N}_{2} \mathrm{O}\left([\mathrm{M}+\mathrm{H}]^{+}\right)$166.1106, found 166.1109. 


\section{5) Alkyl Halide Scope}

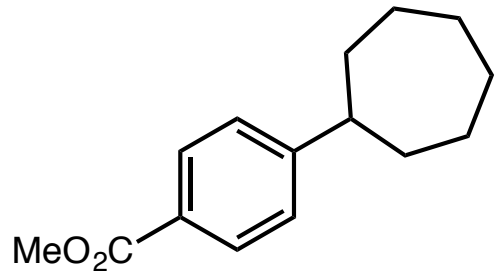

methyl 4-cycloheptylbenzoate (25)

Prepared following the general procedure outlined above using $\operatorname{Ir}\left[\mathrm{dF}\left(\mathrm{CF}_{3}\right) \mathrm{ppy}\right]_{2}$ (dtbbpy)PF 6 (5.6 mg, $5.0 \mu \mathrm{mol}, 0.01$ equiv.), $\mathrm{NiCl}_{2} \bullet$ glyme (0.6 mg, $2.5 \mu \mathrm{mol}, 0.005$ equiv.), 4,4'-di-tert-butyl-2,2'-bipyridine (0.8 mg, $3.0 \mu \mathrm{mol}, 0.006$ equiv.), $\mathrm{Na}_{2} \mathrm{CO}_{3}$ (106.0 mg, $1.00 \mathrm{mmol}, 2.0$ equiv.), TTMSS (154 $\mu \mathrm{L}, 0.50 \mathrm{mmol}, 1.0$ equiv.), bromocycloheptane (103 $\mu \mathrm{L}, 0.75 \mathrm{mmol}, 1.5$ equiv.), methyl 4-bromobenzoate (108 mg, $0.50 \mathrm{mmol}, 1.0$ equiv.), and Toluene/DME (4/1, $5.0 \mathrm{~mL})$. Purification by silica gel column chromatography (EtOAc in hexanes) followed by reverse phase chromatography $\left(\mathrm{MeCN}\right.$ in $\mathrm{H}_{2} \mathrm{O}$ ) yielded the pure product as a clear oil (71 $\mathrm{mg}, 0.31 \mathrm{mmol}, 62 \%$ yield).

${ }^{1}$ H NMR (500 MHz, $\left.\mathbf{C D C l}_{3}\right) \delta 7.94(\mathrm{~d}, J=8.4 \mathrm{~Hz}, 2 \mathrm{H}), 7.25(\mathrm{~d}, J=8.3 \mathrm{~Hz}, 2 \mathrm{H}), 3.89$ (s, $3 \mathrm{H}), 2.71(\mathrm{tt}, J=10.5,3.6 \mathrm{~Hz}, 1 \mathrm{H}), 1.94-1.86(\mathrm{~m}, 2 \mathrm{H}), 1.80(\mathrm{dh}, J=13.3,3.2 \mathrm{~Hz}, 2 \mathrm{H})$, $1.75-1.50(\mathrm{~m}, 4 \mathrm{H})$.

${ }^{13}$ C NMR (125 MHz, $\left.\mathbf{C D C l}_{3}\right) \delta 167.21,155.39,129.75,127.48,126.72,51.95,47.09$, $36.51,27.88,27.23$.

Spectroscopic data matches with previously reported data. ${ }^{9}$ 


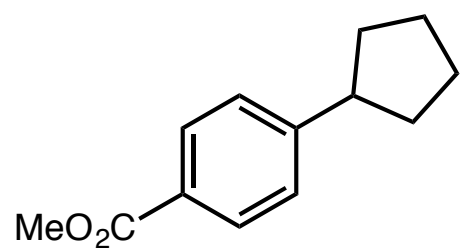

\section{methyl 4-cyclopentylbenzoate (26)}

Prepared following the general procedure outlined above using $\operatorname{Ir}\left[\mathrm{dF}\left(\mathrm{CF}_{3}\right) \text { ppy }\right]_{2}$ (dtbbpy)PF 6 (5.6 mg, $5.0 \mu \mathrm{mol}, 0.01$ equiv.), $\mathrm{NiCl}_{2} \bullet$ glyme (0.6 mg, $2.5 \mu \mathrm{mol}, 0.005$ equiv.), 4,4'-di-tert-butyl-2,2'-bipyridine (0.8 mg, $3.0 \mu \mathrm{mol}, 0.006$ equiv.), $\mathrm{Na}_{2} \mathrm{CO}_{3}$ (106.0 mg, 1.00 mmol, 2.0 equiv.), TTMSS (154 $\mu \mathrm{L}, 0.50 \mathrm{mmol}, 1.0$ equiv.), bromocyclopentane (76 $\mu \mathrm{L}, 0.75 \mathrm{mmol}, 1.5$ equiv.), methyl 4-bromobenzoate (108 $\mathrm{mg}$, $0.50 \mathrm{mmol}, 1.0$ equiv.), and Toluene/DME (4/1, $5.0 \mathrm{~mL})$. Purification by silica gel column chromatography (EtOAc in hexanes) followed by reverse phase chromatography ( $\mathrm{MeCN}$ in $\mathrm{H}_{2} \mathrm{O}$ ) yielded the pure product as a clear oil (70 mg, $0.34 \mathrm{mmol}, 68 \%$ yield).

${ }^{1}$ H NMR (500 MHz, CDCl $)_{3} \delta 7.95(\mathrm{~d}, J=8.1 \mathrm{~Hz}, 2 \mathrm{H}), 7.30(\mathrm{~d}, J=8.1 \mathrm{~Hz}, 2 \mathrm{H}), 3.90$ (s, $3 \mathrm{H}), 3.04(\mathrm{ddd}, J=17.2,9.6,7.6 \mathrm{~Hz}, 1 \mathrm{H}), 2.16-2.01(\mathrm{~m}, 2 \mathrm{H}), 1.88-1.54(\mathrm{~m}, 6 \mathrm{H})$.

${ }^{13}$ C NMR (125 MHz, $\left.\mathbf{C D C l}_{3}\right) \delta 167.20,152.22,129.61,127.62,127.12,77.28,77.03$, $76.77,51.97,45.99,34.52,25.58$.

Spectroscopic data matches with previously reported data. ${ }^{5}$ 


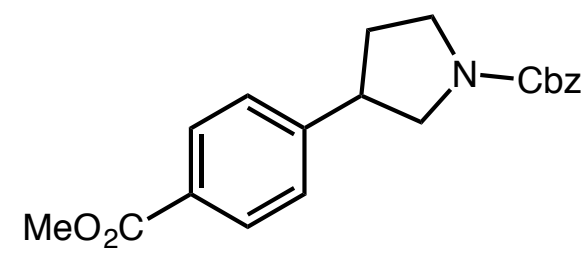

benzyl 3-(4-(methoxycarbonyl)phenyl)pyrrolidine-1-carboxylate (27)

Prepared following the general procedure outlined above using $\operatorname{Ir}\left[\mathrm{dF}\left(\mathrm{CF}_{3}\right) \text { ppy }\right]_{2}$ (dtbbpy)PF 6 (5.6 mg, $10.0 \mu \mathrm{mol}, 0.01$ equiv.), $\mathrm{NiCl}_{2} \bullet$ glyme (0.6 mg, $5.0 \mu \mathrm{mol}, 0.005$ equiv.), 4,4'-di-tert-butyl-2,2'-bipyridine (0.8 mg, $12.0 \mu \mathrm{mol}, 0.006$ equiv.), $\mathrm{Na}_{2} \mathrm{CO}_{3}$ (106.0 mg, 2.0 mmol, 2.0 equiv.), TTMSS (154 $\mu \mathrm{L}, 1.00 \mathrm{mmol}, 1.0$ equiv.), benzyl 3bromopyrrolidine-1-carboxylate $(213 \mathrm{mg}, \quad 0.75 \mathrm{mmol}, \quad 1.5$ equiv.), methyl 4bromobenzoate (108 $\mathrm{mg}, 0.50 \mathrm{mmol}, 1.0$ equiv.), and DME (5.0 mL). Purification by column chromatography yielded the pure product as a clear oil $(136 \mathrm{mg}, 0.40 \mathrm{mmol}, 80 \%$ yield).

${ }^{1}$ H NMR (500 MHz, Acetone-d $\left.)_{6}\right) \delta .97$ (d, $\left.J=8.0 \mathrm{~Hz}, 2 \mathrm{H}\right), 7.49-7.44$ (m, 2H), 7.37 $(\mathrm{dd}, J=13.6,7.6 \mathrm{~Hz}, 5 \mathrm{H}), 5.16-5.11(\mathrm{~m}, 2 \mathrm{H}), 3.95-3.84(\mathrm{~m}, 1 \mathrm{H}), 3.87(\mathrm{~s}, 3 \mathrm{H}), 3.72-$ $3.28(\mathrm{~m}, 4 \mathrm{H}), 2.34(\mathrm{ddt}, J=9.0,6.0,2.9 \mathrm{~Hz}, 1 \mathrm{H}), 2.17-2.08(\mathrm{~m}, 1 \mathrm{H})$.

${ }^{13}$ C NMR (125 MHz, Acetone-d $\left.{ }_{6}\right) \delta$ 167.02, 130.45, 129.62, 129.22, 128.58, 128.55, $128.26,66.85,53.03,52.65,52.26,46.88,46.42,44.88,43.95,33.70,32.78$.

IR (film) $v_{\max } 2951,2883,1697,1416,1277,1106,697 \mathrm{~cm}^{-1}$.

HRMS (ESI-TOF) m/z calcd. for $\mathrm{C}_{20} \mathrm{H}_{22} \mathrm{NO}_{4}\left([\mathrm{M}+\mathrm{H}]^{+}\right) 339.1470$, found 339.1468. 


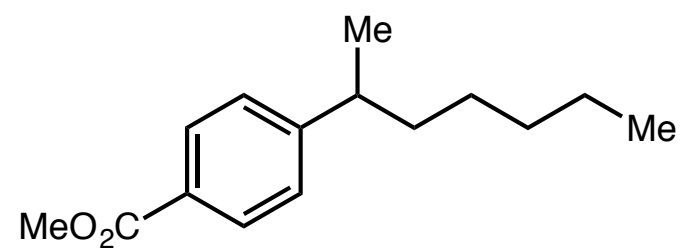

methyl 4-(heptan-2-yl)benzoate (28)

Prepared following the general procedure outlined above using $\operatorname{Ir}\left[\mathrm{dF}\left(\mathrm{CF}_{3}\right) \text { ppy }\right]_{2}$ (dtbbpy)PF 6 (5.6 mg, $5.0 \mu \mathrm{mol}, 0.01$ equiv.), $\mathrm{NiCl}_{2} \bullet$ glyme (0.6 mg, $2.5 \mu \mathrm{mol}, 0.005$ equiv.), 4,4'-di-tert-butyl-2,2'-bipyridine ( $0.8 \mathrm{mg}, 3.0 \mu \mathrm{mol}, 0.006$ equiv.), $\mathrm{Na}_{2} \mathrm{CO}_{3}$ (106.0 mg, $1.00 \mathrm{mmol}, 2.0$ equiv.), TTMSS (154 $\mu \mathrm{L}, 0.50 \mathrm{mmol}, 1.0$ equiv.), 2bromoheptane (118 $\mu \mathrm{L}, 0.75 \mathrm{mmol}, 1.5$ equiv.), methyl 4-bromobenzoate (108 $\mathrm{mg}, 0.50$ mmol, 1.0 equiv.), and Toluene/DME (4/1, $5.0 \mathrm{~mL})$. Purification by silica gel column chromatography (EtOAc in hexanes) followed by reverse phase chromatography (MeCN in $\mathrm{H}_{2} \mathrm{O}$ ) yielded the pure product as a clear oil ( $83 \mathrm{mg}, 0.35 \mathrm{mmol}, 71 \%$ yield).

${ }^{1}$ H NMR (500 MHz, CDCl $) \delta 7.96(\mathrm{~d}, J=8.3 \mathrm{~Hz}, 2 \mathrm{H}), 7.24(\mathrm{~d}, J=8.3 \mathrm{~Hz}, 2 \mathrm{H}), 3.90(\mathrm{~s}$, $3 \mathrm{H}), 2.73$ (h, $J=7.1 \mathrm{~Hz}, 1 \mathrm{H}), 1.59-1.52(\mathrm{~m}, 2 \mathrm{H}), 1.29-1.07$ (m, 9H), $0.88-0.79$ (m, $3 \mathrm{H})$.

${ }^{13}$ C NMR (125 MHz, $\left.\mathbf{C D C l}_{3}\right) \delta 167.22,153.53,129.68,127.76,127.05,77.27,77.02$, $76.77,51.96,40.08,38.14,31.87,27.30,22.56,22.08,14.06$.

IR (film) $v_{\max } 2956,2926,2856,1721,1610,1434,1274,1106 \mathrm{~cm}^{-1}$.

HRMS (ESI-TOF) calcd. for $\mathrm{C}_{15} \mathrm{H}_{22} \mathrm{O}_{2}\left(\left[\mathrm{M}^{*}\right]^{+}\right)$234.1614, found 234.1623 . 


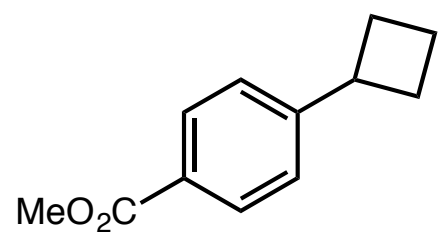

methyl 4-cyclobutylbenzoate (29)

Prepared following the general procedure outlined above using $\operatorname{Ir}\left[\mathrm{dF}\left(\mathrm{CF}_{3}\right) \mathrm{ppy}\right]_{2}$ (dtbbpy)PF 6 (5.6 mg, $5.0 \mu \mathrm{mol}, 0.01$ equiv.), $\mathrm{NiCl}_{2} \bullet$ glyme (0.6 mg, $2.5 \mu \mathrm{mol}, 0.005$ equiv.), 4,4'-di-tert-butyl-2,2'-bipyridine ( $0.8 \mathrm{mg}, 3.0 \mu \mathrm{mol}, 0.006$ equiv.), $\mathrm{Na}_{2} \mathrm{CO}_{3}$ (106.0 mg, $1.00 \mathrm{mmol}, 2.0$ equiv.), TTMSS (154 $\mu \mathrm{L}, 0.50 \mathrm{mmol}, 1.0$ equiv.), bromocyclobutane ( $70 \mu \mathrm{L}, 0.75 \mathrm{mmol}, 1.5$ equiv.), methyl 4-bromobenzoate (108 $\mathrm{mg}$, $0.50 \mathrm{mmol}, 1.0$ equiv.), and DME $(5.0 \mathrm{~mL})$. Purification by column chromatography yielded the pure product as a clear oil ( $58 \mathrm{mg}, 0.31 \mathrm{mmol}, 61 \%$ yield).

${ }^{1}$ H NMR (500 MHz, CDCl $) \delta 7.96(\mathrm{~d}, J=8.4 \mathrm{~Hz}, 2 \mathrm{H}), 7.29-7.24$ (m, 2H), 3.90 (s, $3 \mathrm{H}), 3.60$ (p, $J=8.8 \mathrm{~Hz}, 1 \mathrm{H}$ ), 2.37 (ddt, $J=10.6,7.9,3.8 \mathrm{~Hz}, 2 \mathrm{H}), 2.21-2.12(\mathrm{~m}, 2 \mathrm{H})$, $2.10-1.99(\mathrm{~m}, 1 \mathrm{H}), 1.88(\mathrm{tdd}, J=10.0,5.9,1.8 \mathrm{~Hz}, 1 \mathrm{H})$.

${ }^{13}$ C NMR (125 MHz, $\left.\mathbf{C D C l}_{3}\right) \delta 167.19,151.68,129.58,127.56,126.28,51.98,40.24$, $29.55,18.30$.

Spectroscopic data matches with previously reported data. ${ }^{5}$ 


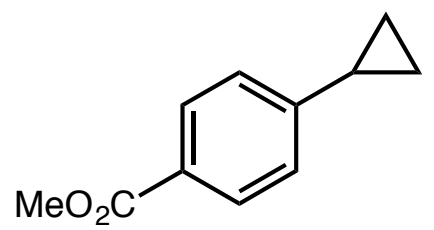

\section{methyl 4-cyclopropylbenzoate (30)}

Prepared following the general procedure outlined above using $\operatorname{Ir}\left[\mathrm{dF}_{(\mathrm{CF}}\right)$ ppy $]_{2}$ (dtbbpy)PF 6 (5.6 mg, $5.0 \mu \mathrm{mol}, 0.01$ equiv.), $\mathrm{NiCl}_{2} \bullet$ glyme (0.6 mg, $2.5 \mu \mathrm{mol}, 0.005$ equiv.), 4,4'-di-tert-butyl-2,2'-bipyridine ( $0.8 \mathrm{mg}, 3.0 \mu \mathrm{mol}, 0.006$ equiv.), $\mathrm{Na}_{2} \mathrm{CO}_{3}$ (106.0 mg, 1.00 mmol, 2.0 equiv.), TTMSS (154 $\mu \mathrm{L}, 0.50 \mathrm{mmol}, 1.0$ equiv.), bromocyclopropane (60 $\mu \mathrm{L}, 0.75 \mathrm{mmol}, 1.5$ equiv.), methyl 4-bromobenzoate (108 $\mathrm{mg}$, $0.50 \mathrm{mmol}, 1.0$ equiv.), and DME (5.0 mL). Purification by column chromatography yielded the pure product as a clear oil (29 $\mathrm{mg}, 0.16 \mathrm{mmol}, 32 \%$ yield).

${ }^{1}$ H NMR (500 MHz, CDCl $\left.)_{3}\right) \quad 7.92(\mathrm{~d}, J=8.3 \mathrm{~Hz}, 2 \mathrm{H}), 7.10(\mathrm{~d}, J=8.4 \mathrm{~Hz}, 2 \mathrm{H}), 1.94$ $(\mathrm{tt}, J=8.4,5.0 \mathrm{~Hz}, 1 \mathrm{H}), 1.08-1.01(\mathrm{~m}, 2 \mathrm{H}), 0.79-0.74(\mathrm{~m}, 2 \mathrm{H})$.

${ }^{13} \mathbf{C}$ NMR (125 MHz, CDCl $\left.\mathbf{3}\right) \delta 167.15,149.98,129.63,127.20,125.32,51.95,15.71$, 10.31 .

Spectroscopic data matches with previously reported data. ${ }^{10}$ 


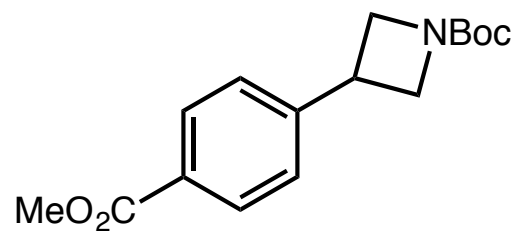

tert-butyl 3-(4-(methoxycarbonyl)phenyl)azetidine-1-carboxylate (31)

Prepared following the general procedure outlined above using $\operatorname{Ir}\left[\mathrm{dF}\left(\mathrm{CF}_{3}\right) \text { ppy }\right]_{2}$ (dtbbpy)PF 6 (5.6 mg, $10.0 \mu \mathrm{mol}, 0.01$ equiv.), $\mathrm{NiCl}_{2} \bullet$ glyme (0.6 mg, $5.0 \mu \mathrm{mol}, 0.005$ equiv.), 4,4'-di-tert-butyl-2,2'-bipyridine ( $0.8 \mathrm{mg}, 12.0 \mu \mathrm{mol}, 0.006$ equiv.), $\mathrm{Na}_{2} \mathrm{CO}_{3}$ (106.0 mg, 2.0 mmol, 2.0 equiv.), TTMSS (154 $\mu \mathrm{L}, 1.00 \mathrm{mmol}, 1.0$ equiv.), tert-butyl 3bromoazetidine-1-carboxylate (177 mg, $0.75 \mathrm{mmol}, 1.5$ equiv.), methyl 4-bromobenzoate (108 mg, $0.50 \mathrm{mmol}, 1.0$ equiv.), and DME (5.0 mL). Purification by column chromatography yielded the pure product as a clear oil (134 mg, $0.460 \mathrm{mmol}, 92 \%$ yield).

${ }^{1}$ H NMR (500 MHz, Acetone-d $) \delta 8.00(\mathrm{~d}, J=8.3 \mathrm{~Hz}, 2 \mathrm{H}), 7.51(\mathrm{~d}, J=8.3 \mathrm{~Hz}, 2 \mathrm{H})$, $4.33(\mathrm{~s}, 2 \mathrm{H}), 3.92(\mathrm{~d}, J=5.3 \mathrm{~Hz}, 3 \mathrm{H}), 3.88(\mathrm{~s}, 3 \mathrm{H}), 1.44(\mathrm{~s}, 9 \mathrm{H})$.

${ }^{13}$ C NMR (125 MHz, Acetone-d $\left.d_{6}\right) \delta 166.99,156.84,149.00,130.59,129.69,127.87$, $79.41,52.29,34.13,28.53$.

IR (film) $v_{\max } 2974,2887,1721,1699,1392,1278,1111,770 \mathrm{~cm}^{-1}$.

HRMS (ESI-TOF) m/z calcd. for $\mathrm{C}_{12} \mathrm{H}_{14} \mathrm{NO}_{4}\left([\mathrm{M}+\mathrm{H}]^{+}\right)$235.0844, found 235.0840. 


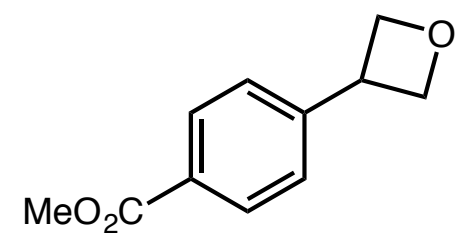

methyl 4-(oxetan-3-yl)benzoate (32)

Prepared following the general procedure outlined above using $\operatorname{Ir}\left[\mathrm{dF}\left(\mathrm{CF}_{3}\right) \mathrm{ppy}\right]_{2}$ (dtbbpy)PF 6 (5.6 mg, $5.0 \mu \mathrm{mol}, 0.01$ equiv.), $\mathrm{NiCl}_{2} \bullet$ glyme (0.6 mg, $2.5 \mu \mathrm{mol}, 0.005$ equiv.), 4,4'-di-tert-butyl-2,2'-bipyridine ( $0.8 \mathrm{mg}, 3.0 \mu \mathrm{mol}, 0.006$ equiv.), $\mathrm{Na}_{2} \mathrm{CO}_{3}$ (106.0 mg, $1.00 \mathrm{mmol}, 2.0$ equiv.), TTMSS (154 $\mu \mathrm{L}, 0.50 \mathrm{mmol}, 1.0$ equiv.), 3bromooxetane ( $63 \mu \mathrm{L}, 0.75 \mathrm{mmol}, 1.5$ equiv.), methyl 4-bromobenzoate (108 mg, 0.50 mmol, 1.0 equiv.), and DME (5.0 mL). Purification by column chromatography yielded the pure product as a clear oil ( $69 \mathrm{mg}, 0.36 \mathrm{mmol}, 72 \%$ yield).

${ }^{1}$ H NMR (500 MHz, CDCl $) \delta 8.04(\mathrm{~d}, J=8.3 \mathrm{~Hz}, 2 \mathrm{H}), 7.47(\mathrm{~d}, J=8.3 \mathrm{~Hz}, 2 \mathrm{H}), 5.10$ (dd, $J=8.3,6.1 \mathrm{~Hz}, 2 \mathrm{H}), 4.77$ (t, $J=6.3 \mathrm{~Hz}, 2 \mathrm{H}), 4.28$ (tt, $J=8.4,6.6 \mathrm{~Hz}, 1 \mathrm{H}), 3.92$ (s, $3 \mathrm{H})$.

${ }^{13}$ C NMR (125 MHz, $\left.\mathbf{C D C l}_{3}\right) \delta 166.84,146.76,130.11,128.97,126.83,78.43,52.15$, 40.26 .

IR (film) $v_{\max } 2953,1875,1720,1611,1435,1277 \mathrm{~cm}^{-1}$.

HRMS (ESI-TOF) $\mathrm{m} / \mathrm{z}$ calcd. for $\mathrm{C}_{11} \mathrm{H}_{13} \mathrm{O}_{3}\left([\mathrm{M}+\mathrm{H}]^{+}\right)$192.0787, found 192.0786. 


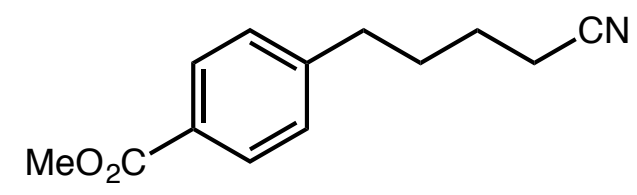

methyl 4-(4-cyanobutyl)benzoate (33)

Prepared following the general procedure outlined above using $\operatorname{Ir}\left[\mathrm{dF}\left(\mathrm{CF}_{3}\right) \text { ppy }\right]_{2}$ (dtbbpy)PF 6 (5.6 mg, $5.0 \mu \mathrm{mol}, 0.01$ equiv.), $\mathrm{NiCl}_{2} \bullet$ glyme (0.6 mg, $2.5 \mu \mathrm{mol}, 0.005$ equiv.), 4,4'-di-tert-butyl-2,2'-bipyridine ( $0.8 \mathrm{mg}, 3.0 \mu \mathrm{mol}, 0.006$ equiv.), $\mathrm{Na}_{2} \mathrm{CO}_{3}$ (106.0 mg, 1.00 mmol, 2.0 equiv.), TTMSS (154 $\mu \mathrm{L}, 0.50 \mathrm{mmol}, 1.0$ equiv.), 5bromopentanenitrile ( $87 \mu \mathrm{L}, 0.75 \mathrm{mmol}, 1.5$ equiv.), methyl 4-bromobenzoate (108 $\mathrm{mg}$, $0.50 \mathrm{mmol}, 1.0$ equiv.), and DME (5.0 mL). Purification by column chromatography yielded the pure product as a clear oil ( $90 \mathrm{mg}, 0.40 \mathrm{mmol}, 82 \%$ yield).

${ }^{1}$ H NMR (500 MHz, CDCl $)_{3} \delta 7.96(\mathrm{~d}, J=8.2 \mathrm{~Hz}, 2 \mathrm{H}), 7.24(\mathrm{~d}, J=8.2 \mathrm{~Hz}, 2 \mathrm{H}), 3.91$ (s, $3 \mathrm{H}), 2.72(\mathrm{t}, J=7.6 \mathrm{~Hz}, 2 \mathrm{H}), 2.36(\mathrm{t}, J=7.0 \mathrm{~Hz}, 2 \mathrm{H}), 1.86-1.77(\mathrm{~m}, 2 \mathrm{H}), 1.73-1.65$ (m, 2H).

${ }^{13}$ C NMR (125 MHz, CDCl 3 ) $\delta$ 167.02, 146.65, 129.85, 128.39, 128.16, 119.46, 52.06, $29.92,24.84,17.10$.

IR (film) $v_{\max } 2951,2868,2246,1716,1610,1434,1276 \mathrm{~cm}^{-1}$.

HRMS (ESI-TOF) m/z calcd. for $\mathrm{C}_{13} \mathrm{H}_{15} \mathrm{NaNO}_{2}\left([\mathrm{M}+\mathrm{Na}]^{+}\right)$240.0995, found 240.0997. 


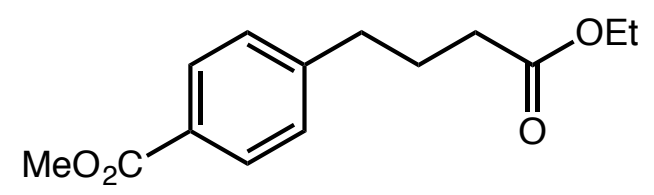

\section{methyl 4-(4-ethoxy-4-oxobutyl)benzoate (34)}

Prepared following the general procedure outlined above using $\operatorname{Ir}\left[\mathrm{dF}\left(\mathrm{CF}_{3}\right) \text { ppy }\right]_{2}$ (dtbbpy)PF 6 (5.6 mg, $5.0 \mu \mathrm{mol}, 0.01$ equiv.), $\mathrm{NiCl}_{2} \bullet$ glyme (0.6 mg, $2.5 \mu \mathrm{mol}, 0.005$ equiv.), 4,4'-di-tert-butyl-2,2'-bipyridine ( $0.8 \mathrm{mg}, 3.0 \mu \mathrm{mol}, 0.006$ equiv.), $\mathrm{Na}_{2} \mathrm{CO}_{3}$ (106.0 mg, $1.00 \mathrm{mmol}, 2.0$ equiv.), TTMSS (154 $\mu \mathrm{L}, 0.50 \mathrm{mmol}, 1.0$ equiv.), ethyl 4bromobutanoate (107 $\mu \mathrm{L}, 146 \mathrm{mg}, 0.75 \mathrm{mmol}, 1.5$ equiv.), methyl 4-bromobenzoate (108 $\mathrm{mg}, 0.50 \mathrm{mmol}, 1.0$ equiv.), and DME (5.0 mL). Purification by column chromatography yielded the pure product as a clear oil (115 mg, $0.46 \mathrm{mmol}, 92 \%$ yield).

${ }^{1}$ H NMR (500 MHz, CDCl $) \delta 7.96(\mathrm{~d}, J=8.3 \mathrm{~Hz}, 2 \mathrm{H}), 7.25(\mathrm{~d}, J=8 \mathrm{~Hz}, 2 \mathrm{H}), 4.13$ (q, $J$ $=7.2 \mathrm{~Hz}, 3 \mathrm{H}), 3.90(\mathrm{~s}, 3 \mathrm{H}), 2.71(\mathrm{t}, J=7.7 \mathrm{~Hz}, 2 \mathrm{H}), 2.32(\mathrm{t}, J=7.5 \mathrm{~Hz}, 2 \mathrm{H}), 2.01-1.90$ $(\mathrm{m}, 2 \mathrm{H}), 1.25(\mathrm{t}, J=7.1 \mathrm{~Hz}, 2 \mathrm{H})$.

${ }^{13}$ C NMR (125 MHz, $\left.\mathbf{C D C l}_{3}\right) \delta 173.42,167.24,147.10,129.89,128.66,128.14,60.52$, $52.17,35.27,33.68,26.32,14.40$.

Spectroscopic data matches with previously reported data. ${ }^{12}$ 


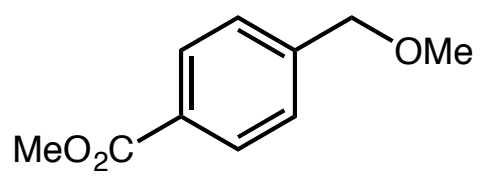

methyl 4-(methoxymethyl)benzoate (35)

Prepared following the general procedure outlined above using $\operatorname{Ir}\left[\mathrm{dF}\left(\mathrm{CF}_{3}\right) \text { ppy }\right]_{2}$ (dtbbpy)PF 6 (5.6 mg, $5.0 \mu \mathrm{mol}, 0.01$ equiv.), $\mathrm{NiCl}_{2} \bullet$ glyme (0.6 mg, $2.5 \mu \mathrm{mol}, 0.005$ equiv.), 4,4'-di-tert-butyl-2,2'-bipyridine (0.8 mg, $3.0 \mu \mathrm{mol}, 0.006$ equiv.), $\mathrm{Na}_{2} \mathrm{CO}_{3}$ (106.0 mg, 1.00 mmol, 2.0 equiv.), TTMSS (154 $\mu \mathrm{L}, 0.50 \mathrm{mmol}, 1.0$ equiv.), methoxymethyl chloride ( $57 \mu \mathrm{L}, 0.75 \mathrm{mmol}, 1.5$ equiv.), methyl 4-bromobenzoate (108 $\mathrm{mg}, 0.50 \mathrm{mmol}, 1.0$ equiv.), and DME (5.0 mL). Purification by column chromatography yielded the pure product as a clear oil (52 mg, $0.29 \mathrm{mmol}, 58 \%$ yield).

${ }^{1}$ H NMR (500 MHz, CDCl $)_{3} \delta 8.02(\mathrm{~d}, J=8.3 \mathrm{~Hz}, 2 \mathrm{H}), 7.40(\mathrm{~d}, J=8.1 \mathrm{~Hz}, 2 \mathrm{H}), 4.51(\mathrm{~s}$, $2 \mathrm{H}), 3.91(\mathrm{~s}, 3 \mathrm{H}), 3.42(\mathrm{~s}, 3 \mathrm{H})$.

${ }^{13}$ C NMR (125 MHz, $\left.\mathbf{C D C l}_{3}\right) \delta 166.98,143.50,129.73,129.36,127.20,77.28,77.03$, $76.77,74.06,58.45,52.13$.

Spectroscopic data matches with previously reported data. ${ }^{11}$ 


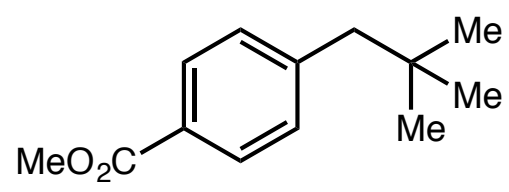

methyl 4-neopentylbenzoate (33)

Prepared following the general procedure outlined above using $\operatorname{Ir}\left[\mathrm{dF}\left(\mathrm{CF}_{3}\right) \text { ppy }\right]_{2}$ (dtbbpy)PF 6 (5.6 mg, $5.0 \mu \mathrm{mol}, 0.01$ equiv.), $\mathrm{NiCl}_{2} \bullet$ glyme (0.6 mg, $2.5 \mu \mathrm{mol}, 0.005$ equiv.), 4,4'-di-tert-butyl-2,2'-bipyridine ( $0.8 \mathrm{mg}, 3.0 \mu \mathrm{mol}, 0.006$ equiv.), $\mathrm{Na}_{2} \mathrm{CO}_{3}$ (106.0 mg, 1.00 mmol, 2.0 equiv.), TTMSS (154 $\mu \mathrm{L}, 0.50 \mathrm{mmol}, 1.0$ equiv.), 1-bromo2,2-dimethylpropane (94 $\mu \mathrm{L}, 113 \mathrm{mg}, 0.75 \mathrm{mmol}, 1.5$ equiv.), methyl 4-bromobenzoate (108 mg, $0.50 \mathrm{mmol}, 1.0$ equiv.), and DME (5.0 mL). Purification by column chromatography yielded the pure product as a clear oil (79 mg, $0.385 \mathrm{mmol}, 77 \%$ yield).

${ }^{1}$ H NMR (500 MHz, Acetone-d d $_{\text {) }} \delta 7.92(\mathrm{~d}, J=8.2 \mathrm{~Hz}, 2 \mathrm{H}), 7.30(\mathrm{~d}, J=8.2 \mathrm{~Hz}, 2 \mathrm{H})$, $3.87(\mathrm{~s}, 3 \mathrm{H}), 2.60(\mathrm{~s}, 2 \mathrm{H}), 0.92(\mathrm{~s}, 9 \mathrm{H})$.

${ }^{13}$ C NMR (125 MHz, Acetone-d $\left.d_{6}\right) \delta 167.26,146.15,131.42,129.57,128.85,52.16$, $50.39,32.36,29.60$.

IR (film) $v_{\max } 2955,2866,1712,1276,1112,1101,733 \mathrm{~cm}^{-1}$.

HRMS (ESI-TOF) $\mathrm{m} / \mathrm{z}$ calcd. for $\mathrm{C}_{13} \mathrm{H}_{19} \mathrm{O}_{2}\left([\mathrm{M}+\mathrm{H}]^{+}\right)$206.1306, found 206.1303. 


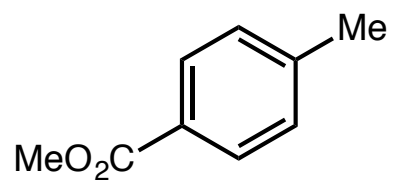

\section{methyl 4-methylbenzoate (37)}

Prepared following the general procedure outlined above using $\operatorname{Ir}\left[\mathrm{dF}\left(\mathrm{CF}_{3}\right) \text { ppy }\right]_{2}$ (dtbbpy)PF 6 (5.6 mg, $5.0 \mu \mathrm{mol}, 0.01$ equiv.), $\mathrm{NiCl}_{2} \bullet$ glyme (0.6 mg, $2.5 \mu \mathrm{mol}, 0.005$ equiv.), 4,4'-di-tert-butyl-2,2'-bipyridine ( $0.8 \mathrm{mg}, 3.0 \mu \mathrm{mol}, 0.006$ equiv.), $\mathrm{Na}_{2} \mathrm{CO}_{3}$ (106 mg, 1.00 mmol, 2.0 equiv.), $\mathrm{LiBr}(65.0 \mathrm{mg}, 0.75 \mathrm{mmol}, 1.5$ equiv.), TTMSS (154 $\mu \mathrm{L}$, $0.50 \mathrm{mmol}, 1.0$ equiv.), methyl 4-methylbenzenesulfonate (113 $\mu \mathrm{L}, 140 \mathrm{mg}, 0.75 \mathrm{mmol}$, 1.5 equiv.), methyl 4-bromobenzoate (108 mg, $0.50 \mathrm{mmol}, 1.0$ equiv.), and DME (5.0 $\mathrm{mL})$. Purification by column chromatography (C18 column) yielded the pure product as a clear oil (48 mg, $0.31 \mathrm{mmol}, 62 \%$ yield).

Additionally, use of $\mathrm{MeBr}$ in DME afforded the product as a clear oil (56 $\mathrm{mg}, 0.375$ mmol, $75 \%$ yield).

${ }^{1}$ H NMR (500 MHz, CDCl $) \delta 7.93(\mathrm{~d}, J=8.3 \mathrm{~Hz}, 2 \mathrm{H}), 7.24(\mathrm{~d}, J=8.0 \mathrm{~Hz}, 2 \mathrm{H}), 3.90$ (s, $3 \mathrm{H}), 2.41(\mathrm{~s}, 3 \mathrm{H})$.

${ }^{13}$ C NMR (125 MHz, $\left.\mathbf{C D C l}_{3}\right) \delta$ 167.20, 143.56, 129.60, 129.08, 127.42, 51.97, 21.68.

Spectroscopic data matches with previously reported data. ${ }^{13}$ 


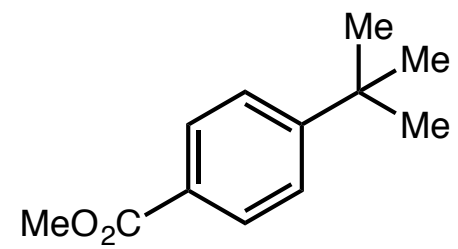

methyl 4-(tert-butyl)benzoate (38)

Prepared following the general procedure outlined above using $\operatorname{Ir}\left[\mathrm{dF}\left(\mathrm{CF}_{3}\right) \text { ppy }\right]_{2}$ (dtbbpy)PF 6 (5.6 mg, $5.0 \mu \mathrm{mol}, 0.01$ equiv.), $\mathrm{NiCl}_{2} \bullet$ glyme (0.6 mg, $2.5 \mu \mathrm{mol}, 0.005$ equiv.), 4,4'-di-tert-butyl-2,2'-bipyridine ( $0.8 \mathrm{mg}, 3.0 \mu \mathrm{mol}, 0.006$ equiv.), $\mathrm{Na}_{2} \mathrm{CO}_{3}$ (106 mg, 1.00 mmol, 2.0 equiv.), TTMSS (213 $\mu \mathrm{L}, 0.75$ mmol, 1.5 equiv.), 2-bromo-2methylpropane ( $84 \mu \mathrm{L}, 0.75 \mathrm{mmol}, 1.5$ equiv.), methyl 4-bromobenzoate (108 mg, 0.50 mmol, 1.0 equiv.), and 1,4-dioxanes $(5.0 \mathrm{~mL})$. The reaction was allowed to run for 48 hours. Purification by column chromatography the pure product as a clear oil $(39 \mathrm{mg}$, $0.26 \mathrm{mmol}, 52 \%$ yield).

${ }^{1}$ H NMR (500 MHz, CDCl $)_{3} \delta 7.97(\mathrm{~d}, J=8.7 \mathrm{~Hz}, 2 \mathrm{H}), 7.45(\mathrm{~d}, J=9.0 \mathrm{~Hz}, 2 \mathrm{H}), 3.90(\mathrm{~s}$, $3 \mathrm{H}), 1.35(\mathrm{~s}, 9 \mathrm{H})$.

${ }^{13} \mathbf{C}$ NMR (125 MHz, $\left.\mathbf{C D C l}_{3}\right) \delta 167.30,156.71,129.60,127.52,125.50,51.97,35.19$, 31.31 .

Spectroscopic data matches with previously reported data. ${ }^{14}$ 


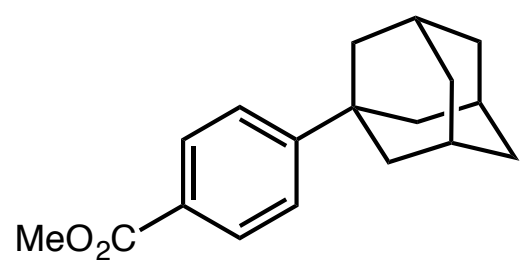

methyl 4-(adamantan-1-yl)benzoate (39)

Prepared following the general procedure outlined above using $\operatorname{Ir}\left[\mathrm{dF}\left(\mathrm{CF}_{3}\right) \text { ppy }\right]_{2}$ (dtbbpy)PF 6 (5.6 mg, $5.0 \mu \mathrm{mol}, 0.01$ equiv.), $\mathrm{NiCl}_{2} \bullet$ glyme (0.6 mg, $2.5 \mu \mathrm{mol}, 0.005$ equiv.), 4,4'-di-tert-butyl-2,2'-bipyridine ( $0.8 \mathrm{mg}, 3.0 \mu \mathrm{mol}, 0.006$ equiv.), $\mathrm{Na}_{2} \mathrm{CO}_{3}$ (106 mg, 1.00 mmol, 2.0 equiv.), TTMSS (213 $\mu \mathrm{L}, 0.75$ mmol, 1.5 equiv.), 1bromoadamantane (161 mg, $0.75 \mathrm{mmol}, 1.5$ equiv.), methyl 4-bromobenzoate (108 $\mathrm{mg}$, $0.50 \mathrm{mmol}, 1.0$ equiv.), and 1,4-dioxanes $(5.0 \mathrm{~mL})$. The reaction was allowed to run for 24 hours. Purification by column chromatography the pure product as white solide (84 $\mathrm{mg}, 0.310 \mathrm{mmol}, 62 \%$ yield).

${ }^{1}$ H NMR (500 MHz, $\left.\mathbf{C D C l}_{3}\right) \delta 7.98(\mathrm{~d}, J=8.5 \mathrm{~Hz}, 2 \mathrm{H}), 7.43(\mathrm{~d}, J=8.5 \mathrm{~Hz}, 2 \mathrm{H}), 3.90(\mathrm{~s}$, $3 \mathrm{H}), 2.16-2.06(\mathrm{~m}, 3 \mathrm{H}), 1.93(\mathrm{~s}, 6 \mathrm{H}), 1.78(\mathrm{q}, J=12.1 \mathrm{~Hz}, 6 \mathrm{H})$.

${ }^{13}$ C NMR (125 MHz, $\left.\mathbf{C D C l}_{3}\right) \delta 167.36,156.79,129.60,127.48,125.10,52.11,43.03$, $36.81,28.94$.

Spectroscopic data matches with previously reported data. ${ }^{15}$ 


\section{6) Cyclic Voltammetry Data}

Cyclic voltammetry was performed using a CHI 1140A potentiostat, a glassy carbon working electrode, a platinum mesh counter electrode, and a $\mathrm{Ag} / \mathrm{AgCl}$ reference electrode. Samples were prepared with a substrate concentration of $0.01 \mathrm{M}$ in a $0.1 \mathrm{M}$ tetrabutylammonium tetrafluoroborate in dimethoxyethane electrolyte solution and sparged with $\mathrm{N}_{2}$ for 15 minutes. Data was collected with a scan rate of $0.1 \mathrm{~V} / \mathrm{s}$.

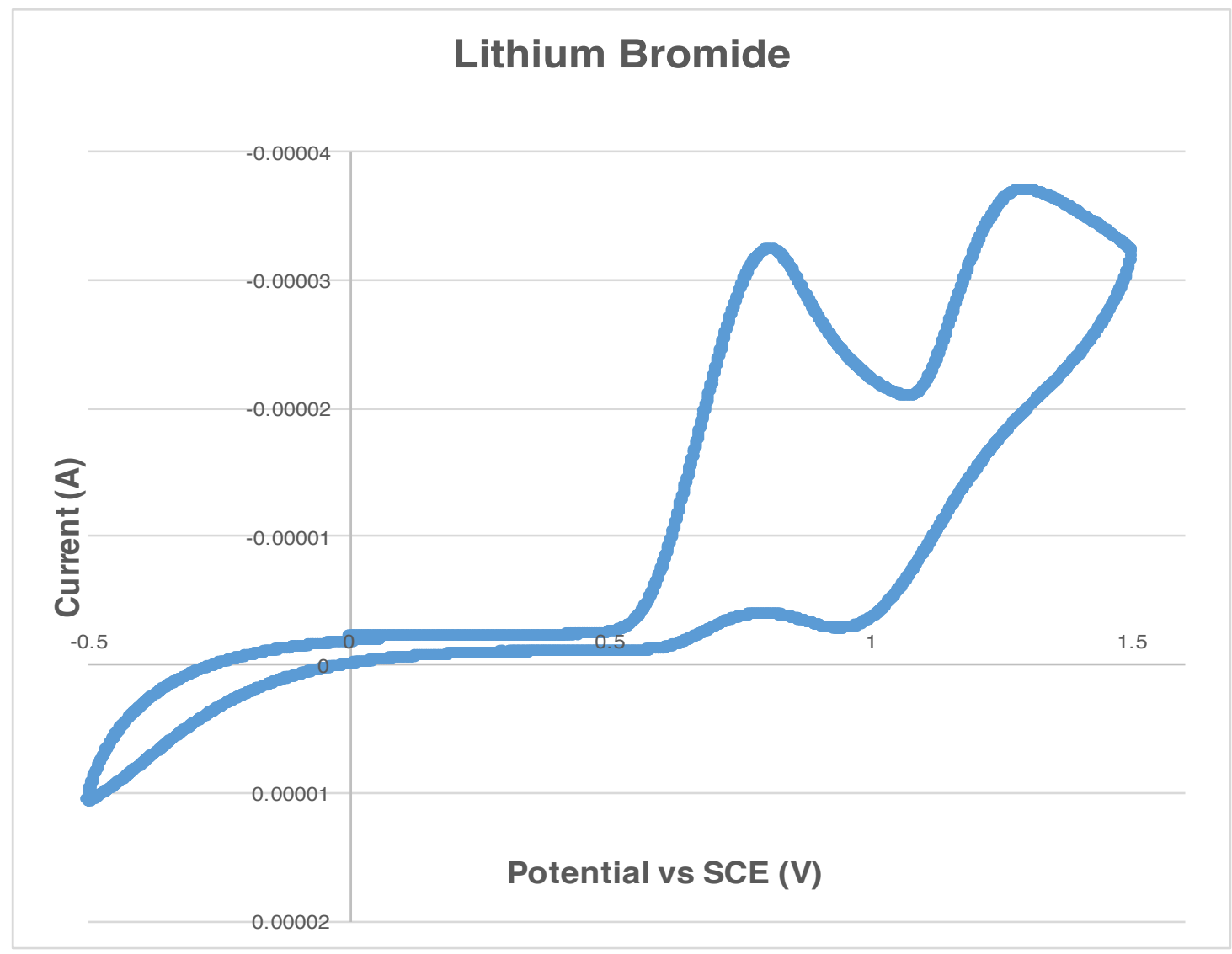




\section{7) Stern-Volmer Fluorescence Quenching Experiments}

Fluorescence quenching experiments were performed on an Agilent Cary Eclipse Fluorescence Spectrophotometer. In a typical experiment, a $2.5 \mu \mathrm{M}$ solution of $\operatorname{Ir}\left[\mathrm{dF}\left(\mathrm{CF}_{3}\right) \text { ppy }\right]_{2}(\mathrm{dtbbpy}) \mathrm{PF}_{6}$ in $\mathrm{DME}$ was added to the appropriate amount of quencher in a screw-top $1.0 \mathrm{~cm}$ quartz cuvette. After degassing by bubbling a stream of nitrogen for 10 minutes, the emission of the sample was collected. All solutions were excited at $\lambda=$ $380 \mathrm{~nm}$ (absorption maximum of the photocatalyst) and the emission intensity at $474 \mathrm{~nm}$ was observed (emission maximum). Plots were constructed according to the SternVolmer equation $\mathrm{I}_{0} / \mathrm{I}=1+\mathrm{kq}_{0}[\mathrm{Q}]{ }^{16}$

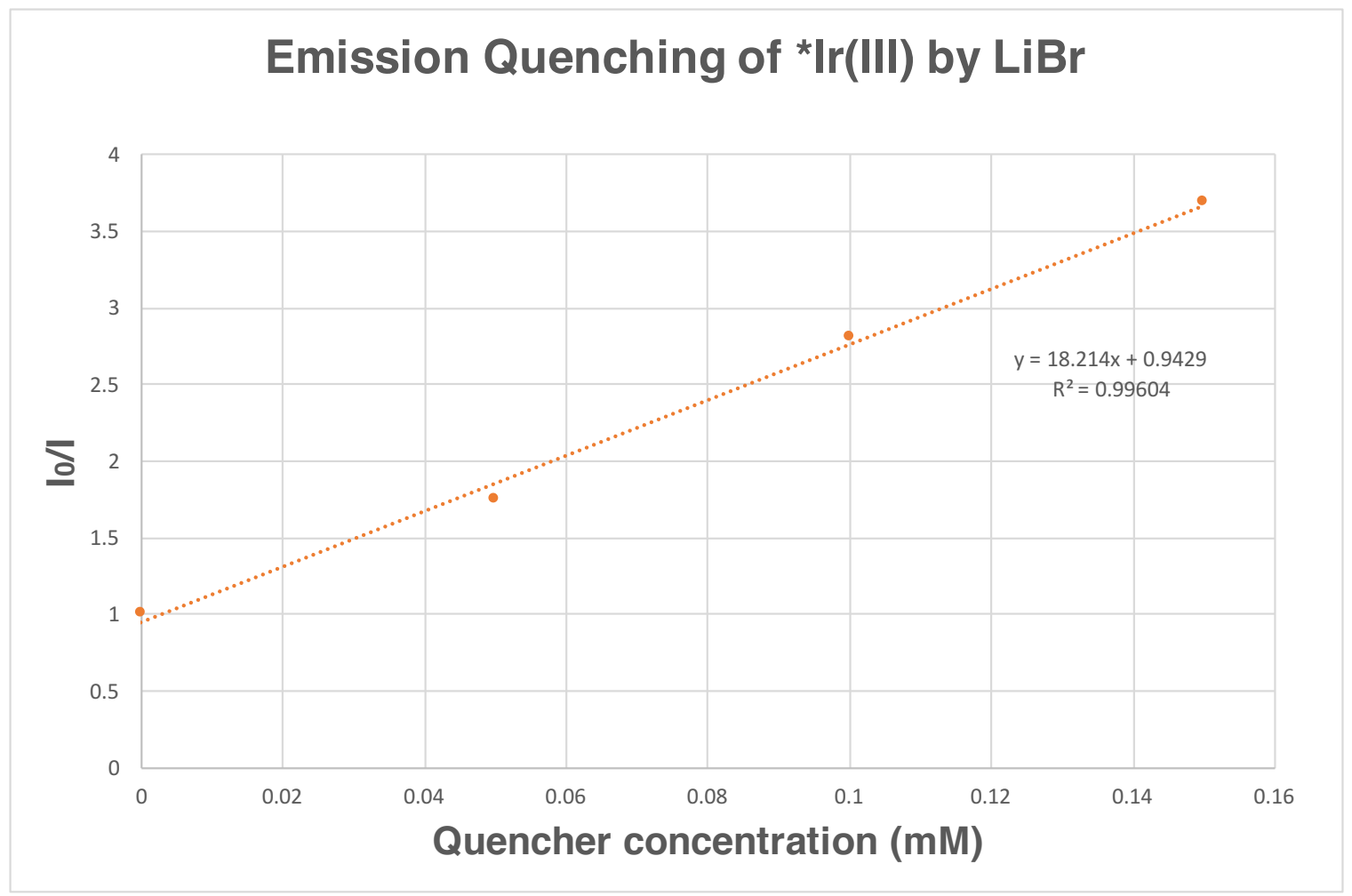




\section{8) Procedure for Investigating Other Reductants}

Reactions in Table 3 were run according to the following procedure:

To an $8 \mathrm{~mL}$ vial equipped with a stir bar was added the indicated photocatalyst $(2.5$ umol, 0.01 equiv.), methyl 4-bromo benzoate (54 mg, $0.25 \mathrm{mmol}, 1$ equiv.), 4bromotetrahydropyran $(42 \mu \mathrm{L}, 0.375 \mathrm{mmol}, 1.5$ equiv.), the indicated reductant $(0.25$ mmol, 1.0 equiv), and anhydrous sodium carbonate (53 $\mathrm{mg}, 0.5 \mathrm{mmol}, 2$ equiv.). The vial was sealed and placed under nitrogen before $2 \mathrm{~mL}$ of solvent was added. To a separate vial was added $\mathrm{NiCl}_{2} \bullet$ glyme (2.8 $\mathrm{mg}, 0.013 \mathrm{mmol}, 0.05$ equiv.) and 4,4'-di-tert-butyl2,2'-bipyridine (3.4 mg, $0.013 \mathrm{mmol}, 0.05$ equiv). The catalyst vial was sealed, purged with nitrogen then to it was added $1 \mathrm{~mL}$ of solvent. The precatalyst solution was sonicated or stirred for 5 minutes, after which, $0.1 \mathrm{~mL}$ of the solution $(0.5 \mathrm{~mol} \%$ catalyst, $1.25 \mu \mathrm{mol}, 0.005$ equv.) was syringed into the reaction vessel. The solution was degassed by sparging with nitrogen while stirring for 10 minutes before sealing with Parafilm. The reaction was stirred and irradiated with a $34 \mathrm{~W}$ blue LED lamp (7 cm away, with cooling fan to keep the reaction temperature at $25^{\circ} \mathrm{C}$ ) for 6 hours. The reaction was quenched by exposure to air. Mesitylene (internal standard, $35 \mu \mathrm{L}, 0.250 \mathrm{mmol}, 1.0$ equiv.) was added then the reaction mixture was analyzed by ${ }^{1} \mathrm{H}$ NMR. 


\section{9) References}

1) Perrin, D. D. \& Armarego, W. L. F. Purification of Laboratory Chemicals (Pergamon Press: Oxford, 1988) ed 3.

2) Lowry, M. S., Goldsmith, J. I., Slinker, J. D., Rohl, R., Pascal, R. A., Malliaras, G. G. \& Bernhard, S. Chem. Mater. 2005, 17, 5712.

3) Pangborn, A. B., Giardello, M. A., Grubbs, R. H., Rosen, R. K. \& Timmers, F. J. Organometallics 1996, 15, 1518.

4) Still, W. C., Kahn, M. \& Mitra, A. J. Org. Chem. 1978, 43, 2923.

5) Primer, D. N.; Karakaya, I.; Tellis, J. C.; Molander, G. A. J. Am. Chem. Soc. 2015, $137,2195$.

6) González-Bobes, F.; Fu, G. C. J. Am. Chem. Soc. 2006, 128, 5360.

7) Allwood, D. M.; Blakemore, D. C.; Brown, A. D.; Ley, S. V. J. Org. Chem. 2014, 79, 328.

8) Molander, G. A.; Traister, K. M.; O’Neill, B. T. J. Org. Chem. 2014, 79, 5771.

9) Hatakeyama, T.; Hashimoto, T.; Kondo, Y.; Fujiwara, Y.; Seike, H.; Takaya, H.;

Tamada, Y.; Ono, T.; Nakamura, M. J. Am. Chem. Soc. 2010, 132, 10674.

10) Zhang, M.; Cui, X.; Chen, X.; Wang, L.; Li, J.; Wu, Y.; Hou, L.; Wu, Y. Tetrahedron 2012, 68, 900 .

11) Strazzolini, P.; Runcio, A. Eur. J. Org. Chem. 2003, 526.

12) Dohle, W.; Lindsay, D. M.; Knochel, P. Org. Lett. 2001, 3, 2871.

13) Behera, H.; Ramkumar, V.; Madhavan, N. Chem. Eur. J. 2015, $21,10179$.

14) Bosset, C.; Coffinier, R.; Peixoto, P. A.; El Assal, M.; Miqueu, K.; Sotiropoulos, J.M.; Pouységu, L.; Quideau, S. Angew. Chem. Int. Ed. 2014, 53, 9860.

15) Zhang, X.; Yang, C. Adv. Synth. Catal. 2015, 357, 2721.

16) N. J. Turro, Modern Molecular Photochemistry, Benjamin/Cummings: Menlo Park, CA, 1978 


\section{0) Spectral Data for Alkyl-Aryl Compounds}

\section{${ }^{1} \mathrm{H}$ and ${ }^{13} \mathrm{C}$ Spectra for Novel Compounds}

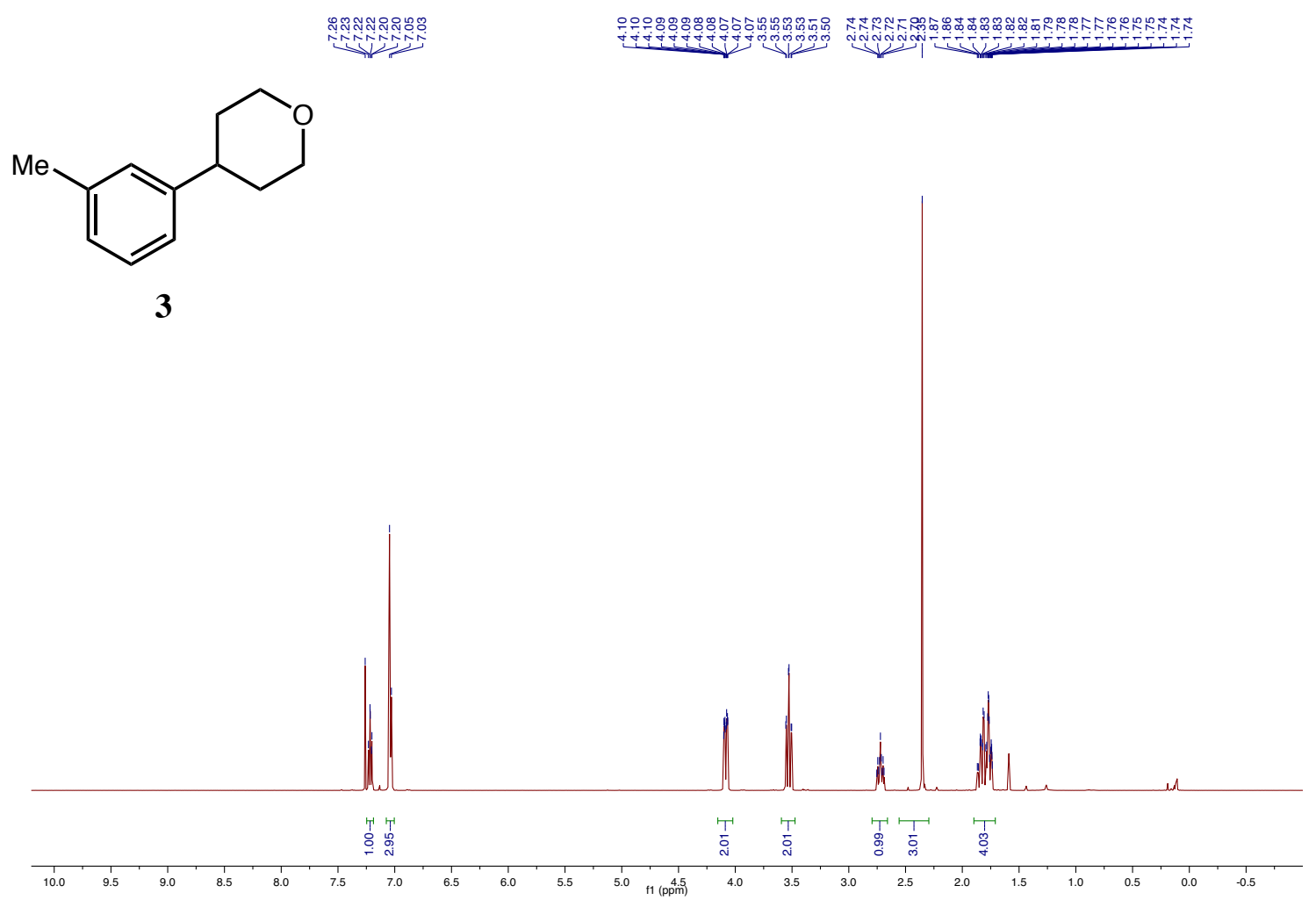




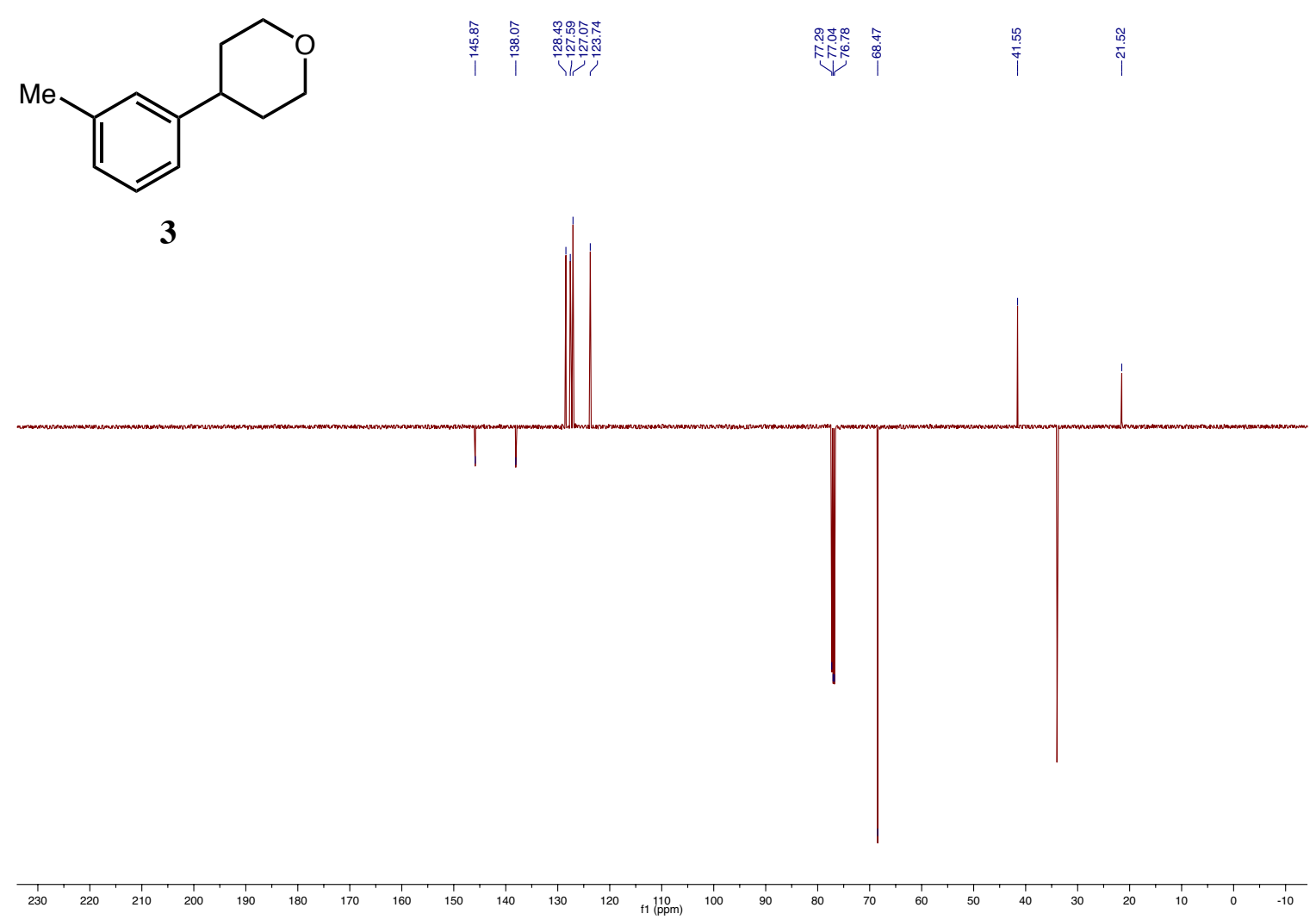




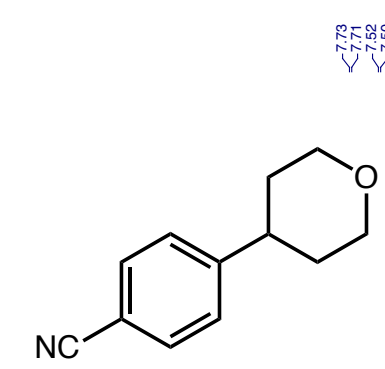

7

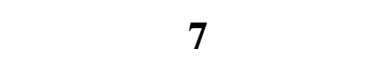

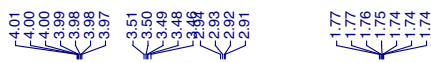
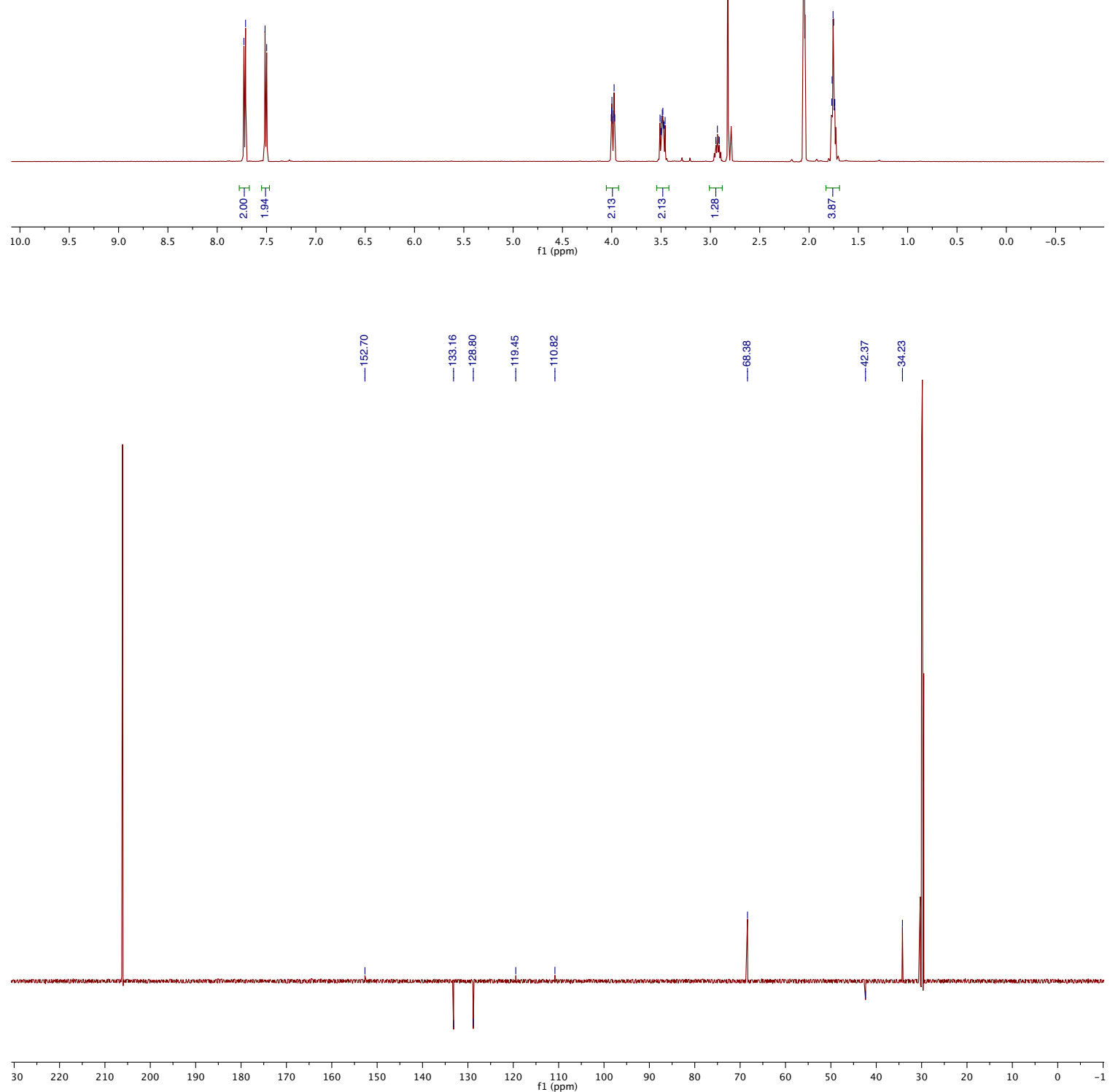


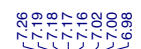

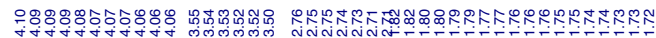

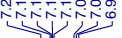
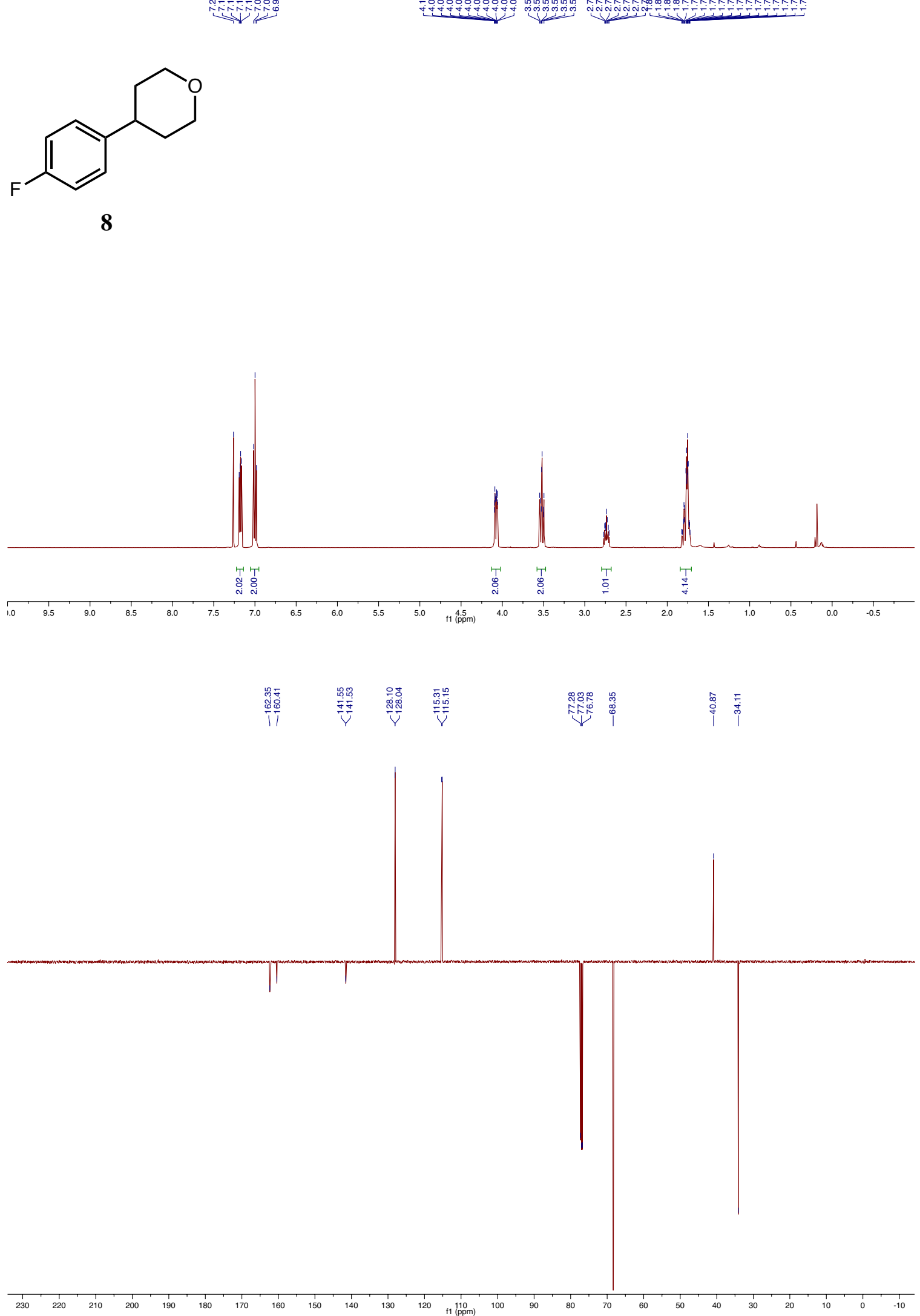
${ }^{19}$ F NMR<smiles>Fc1ccc(C2CCOCC2)cc1</smiles> 

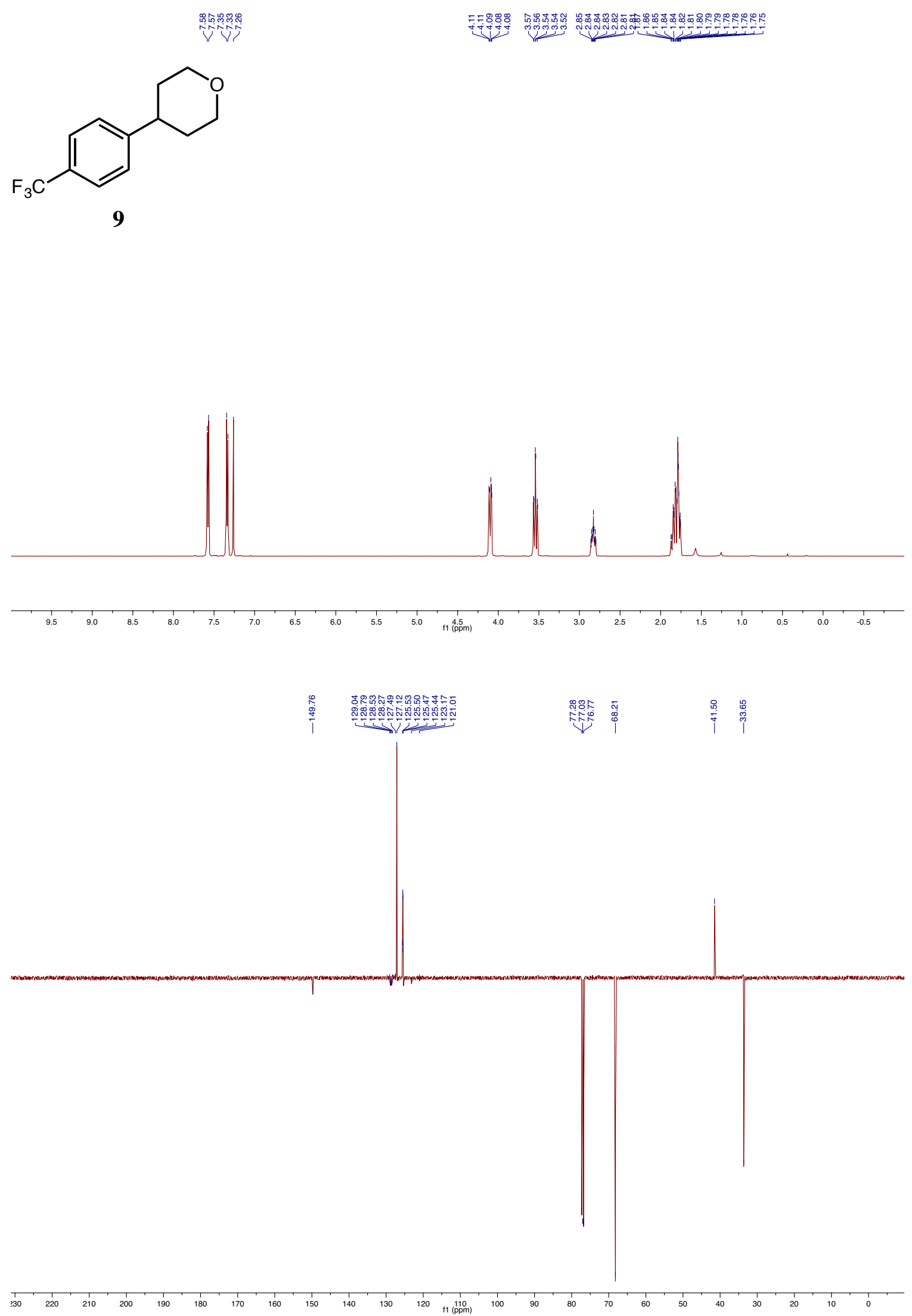
${ }^{19}$ F NMR

? 


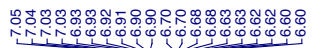

$\underbrace{\overline{2}}$<smiles>Nc1ccccc1C1CCOCC1</smiles>

10
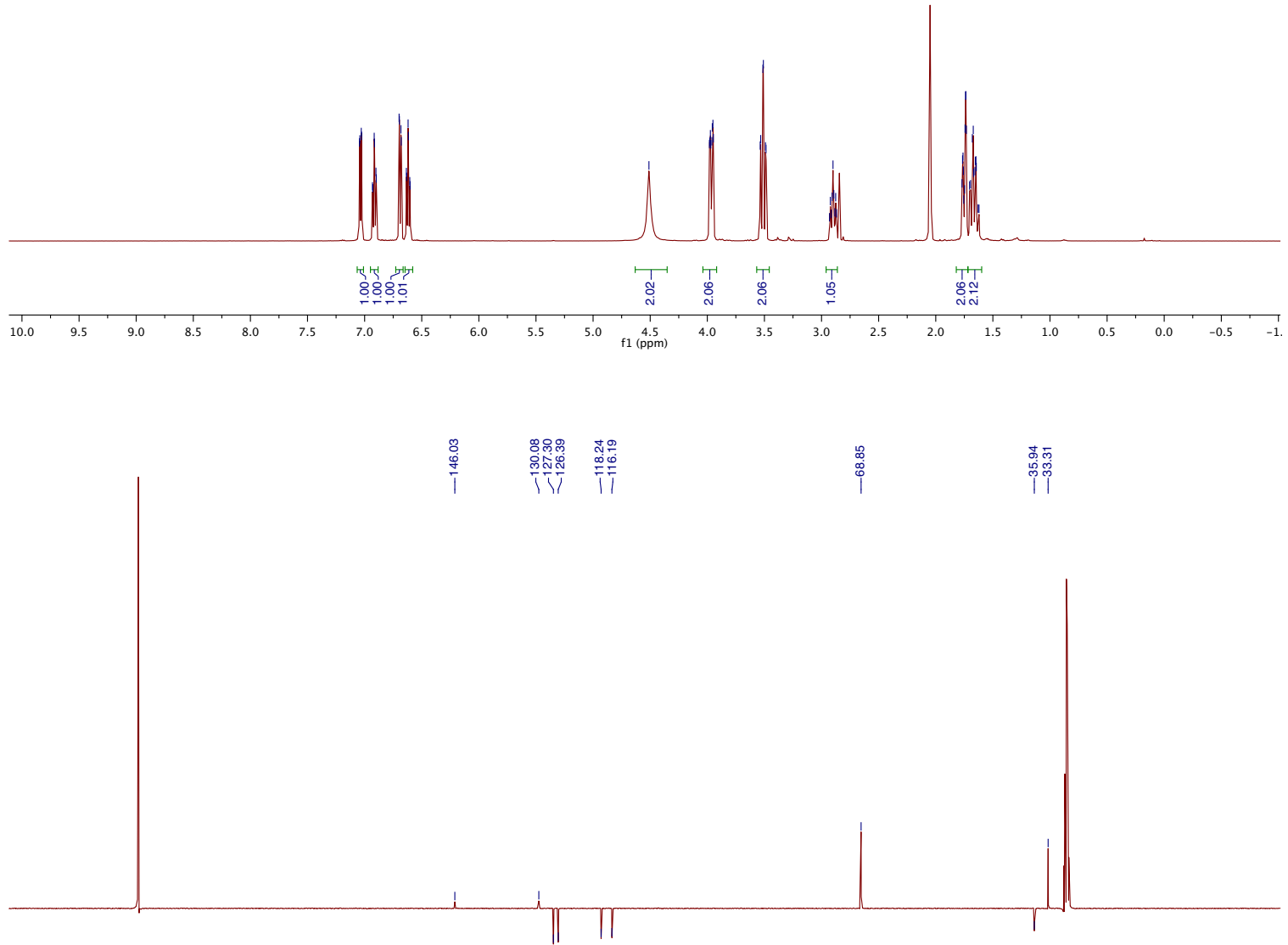

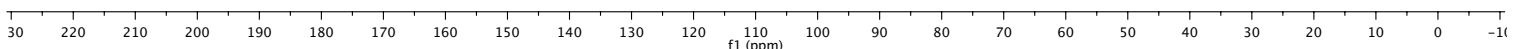




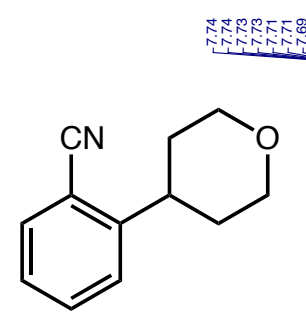

11

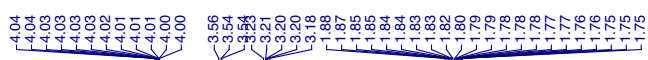

(1) 

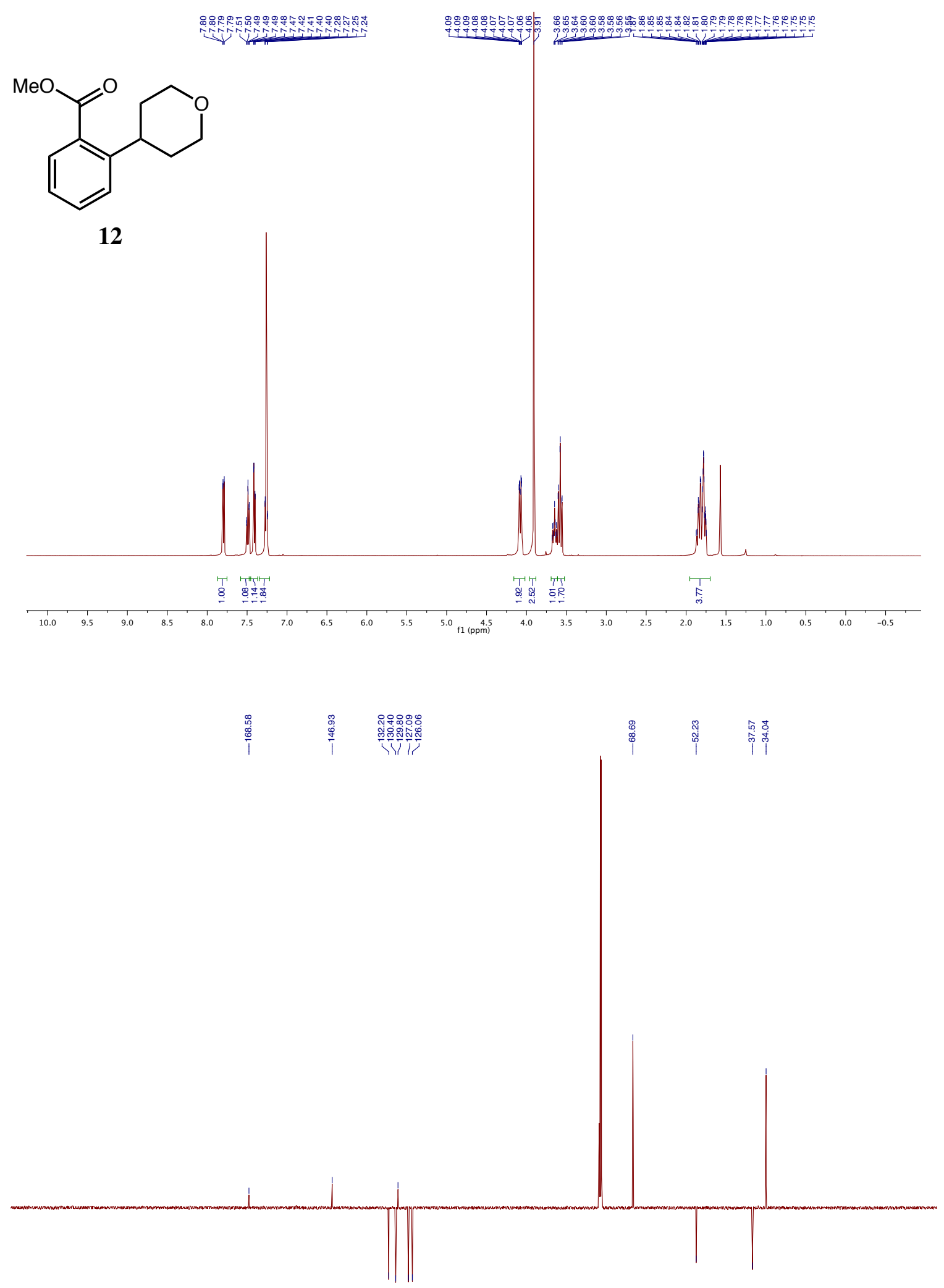

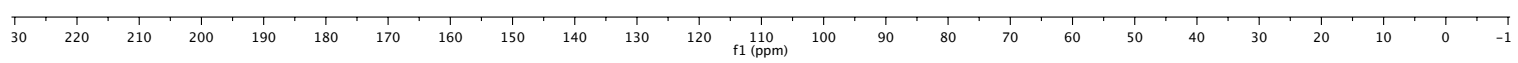


Бே.

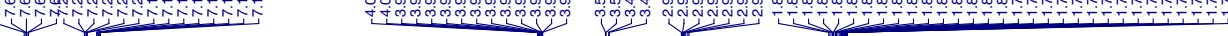<smiles>c1ccc(C2CCOCC2)nc1</smiles>

13

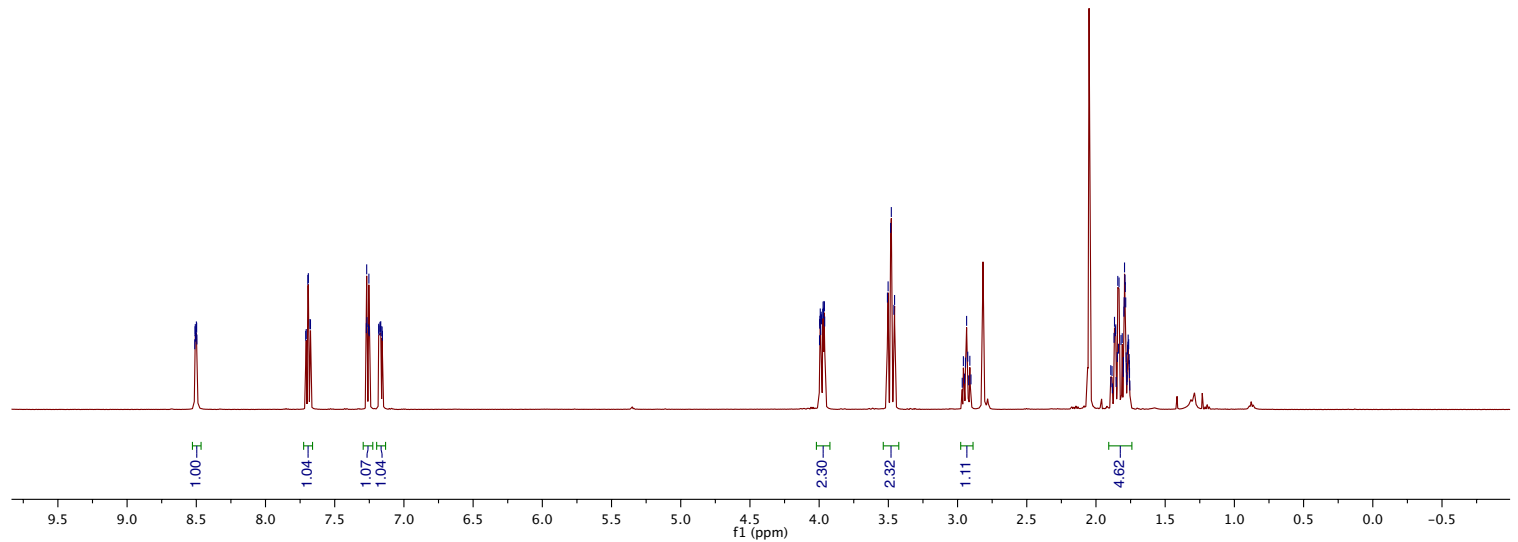

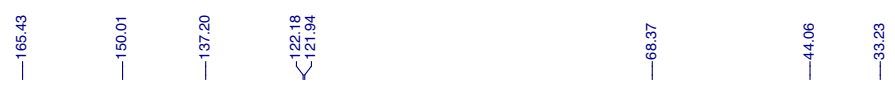

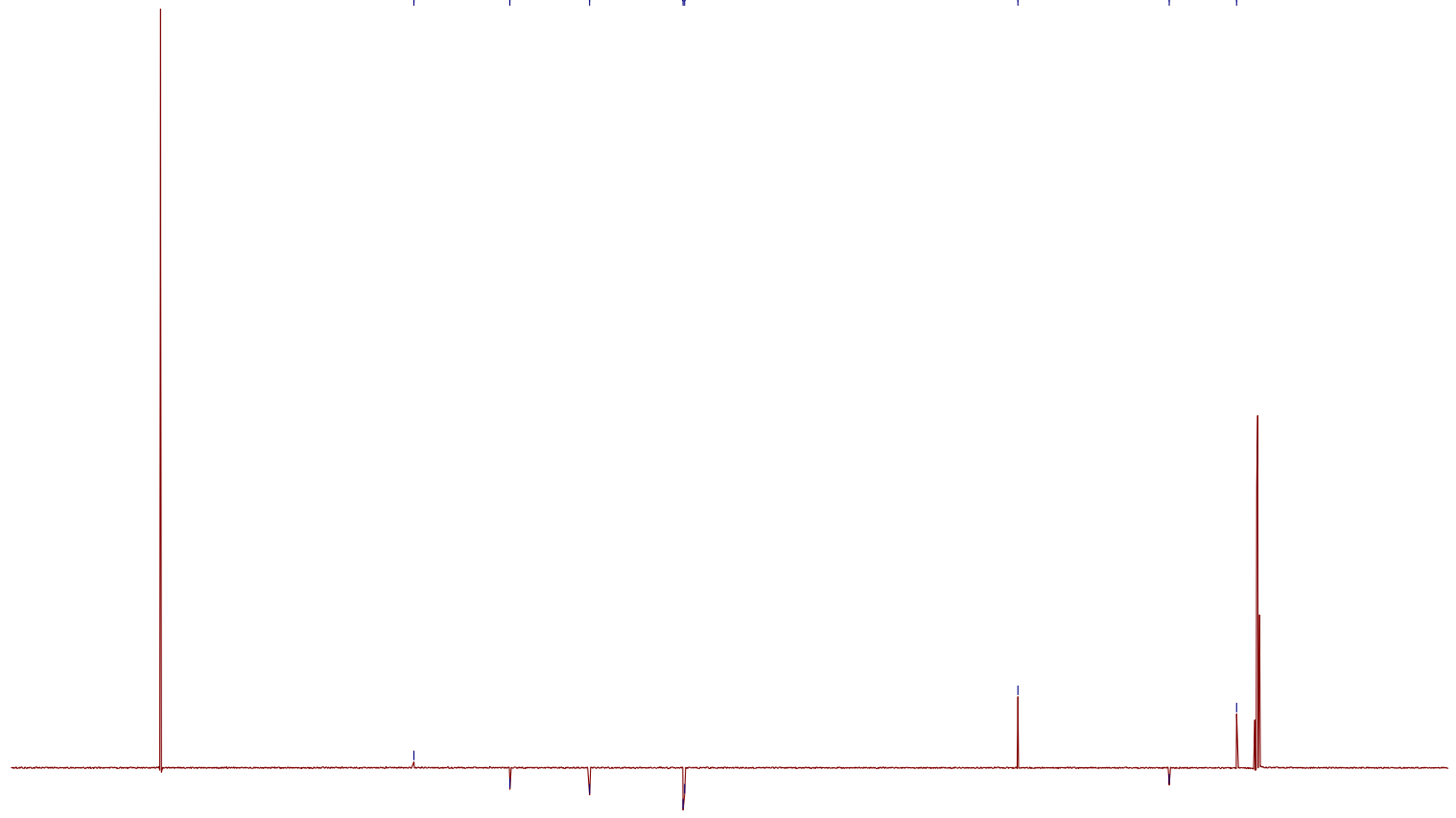

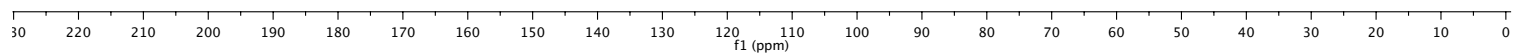




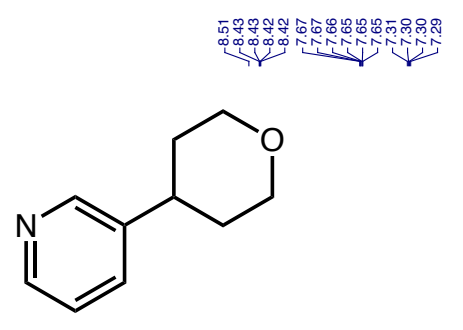

$\underbrace{8 \text { 8.8. }}$

14
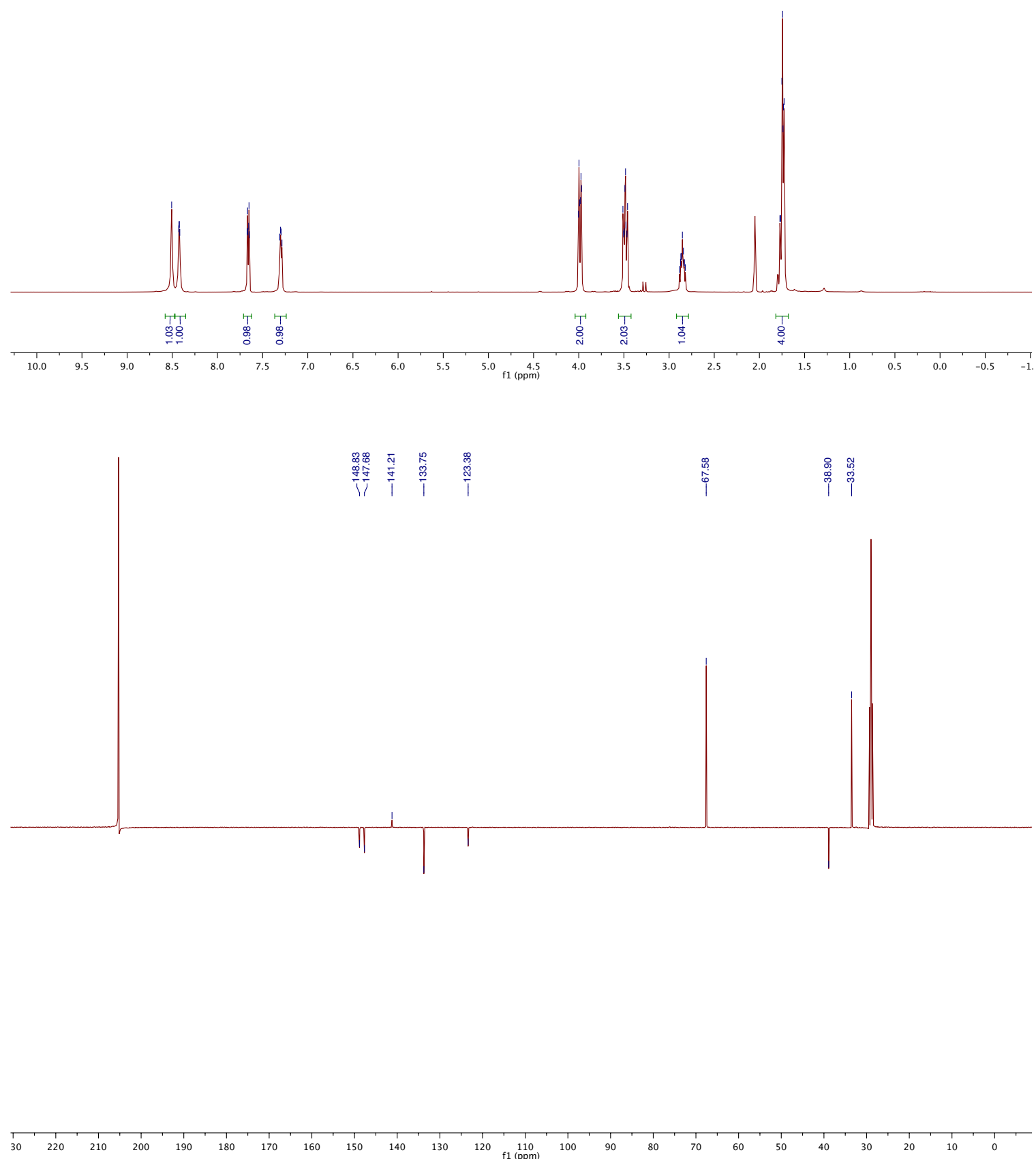


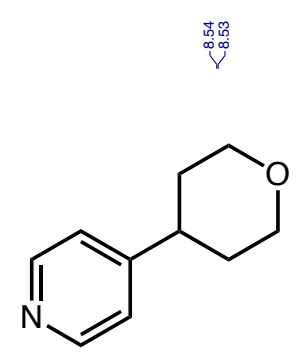

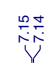

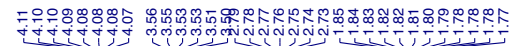

15
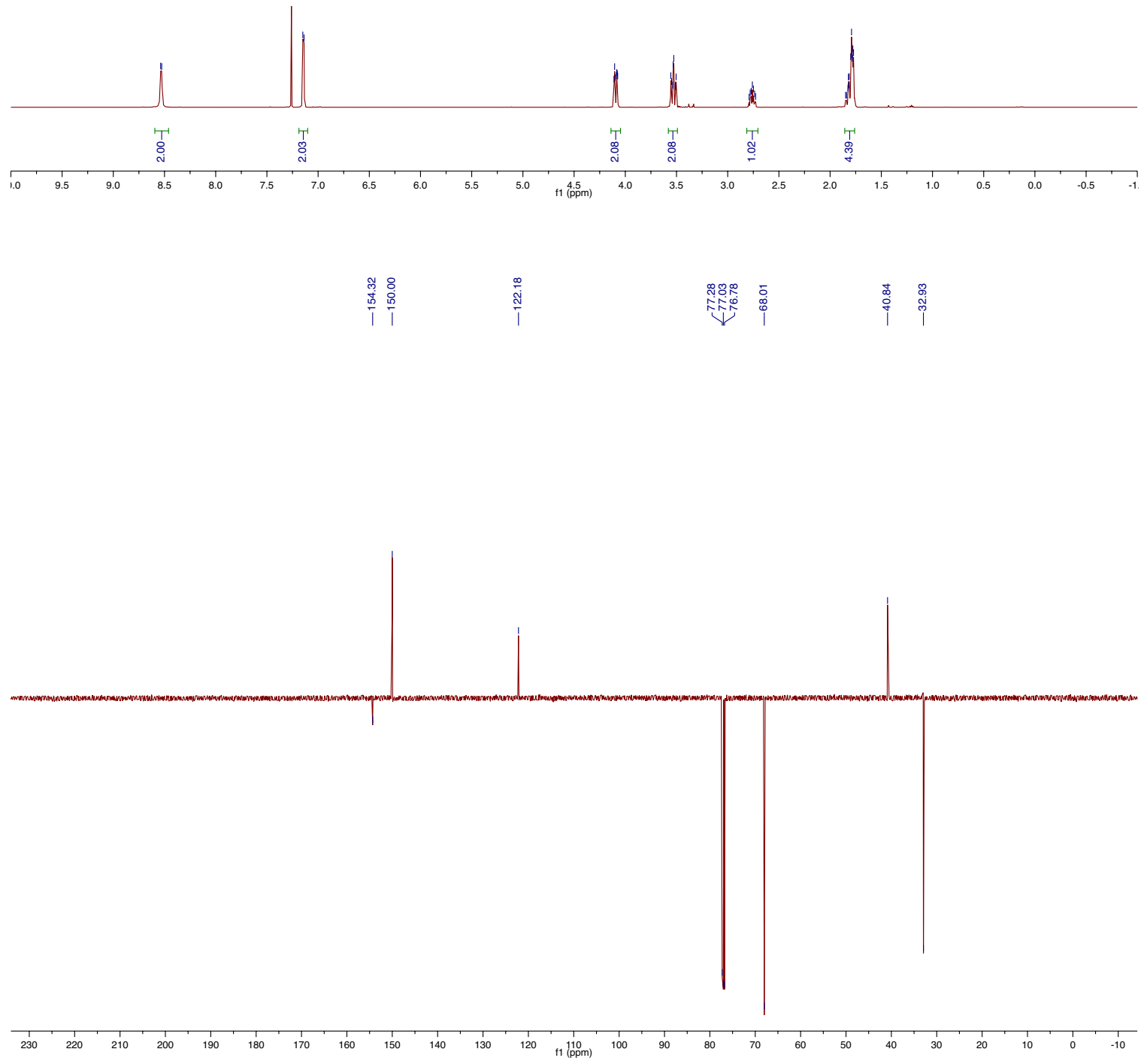
<smiles>Cc1cc(C2CCOCC2)ccn1</smiles>

16

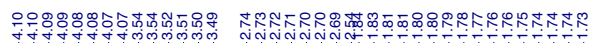
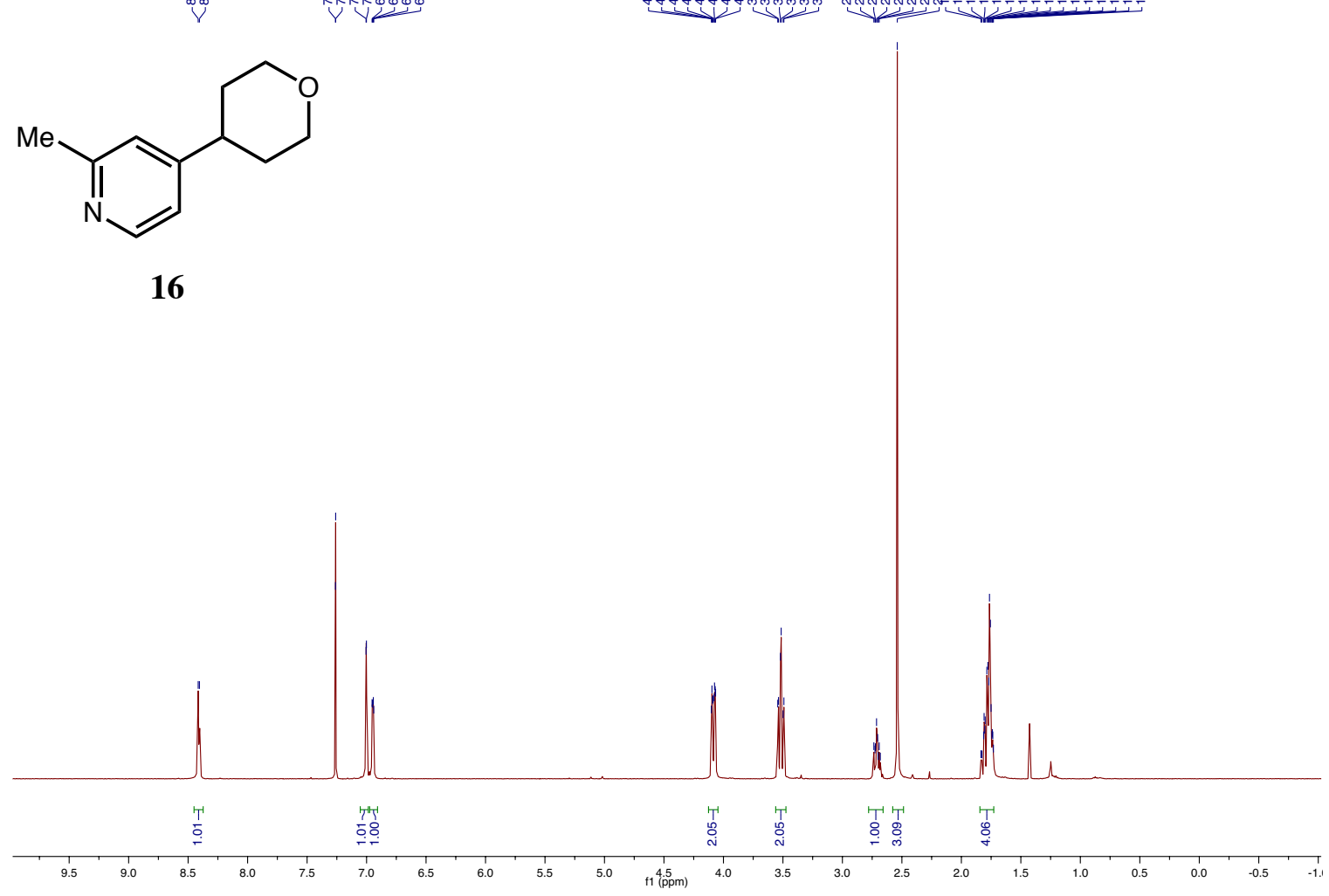
\#II
|

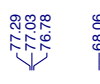

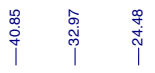
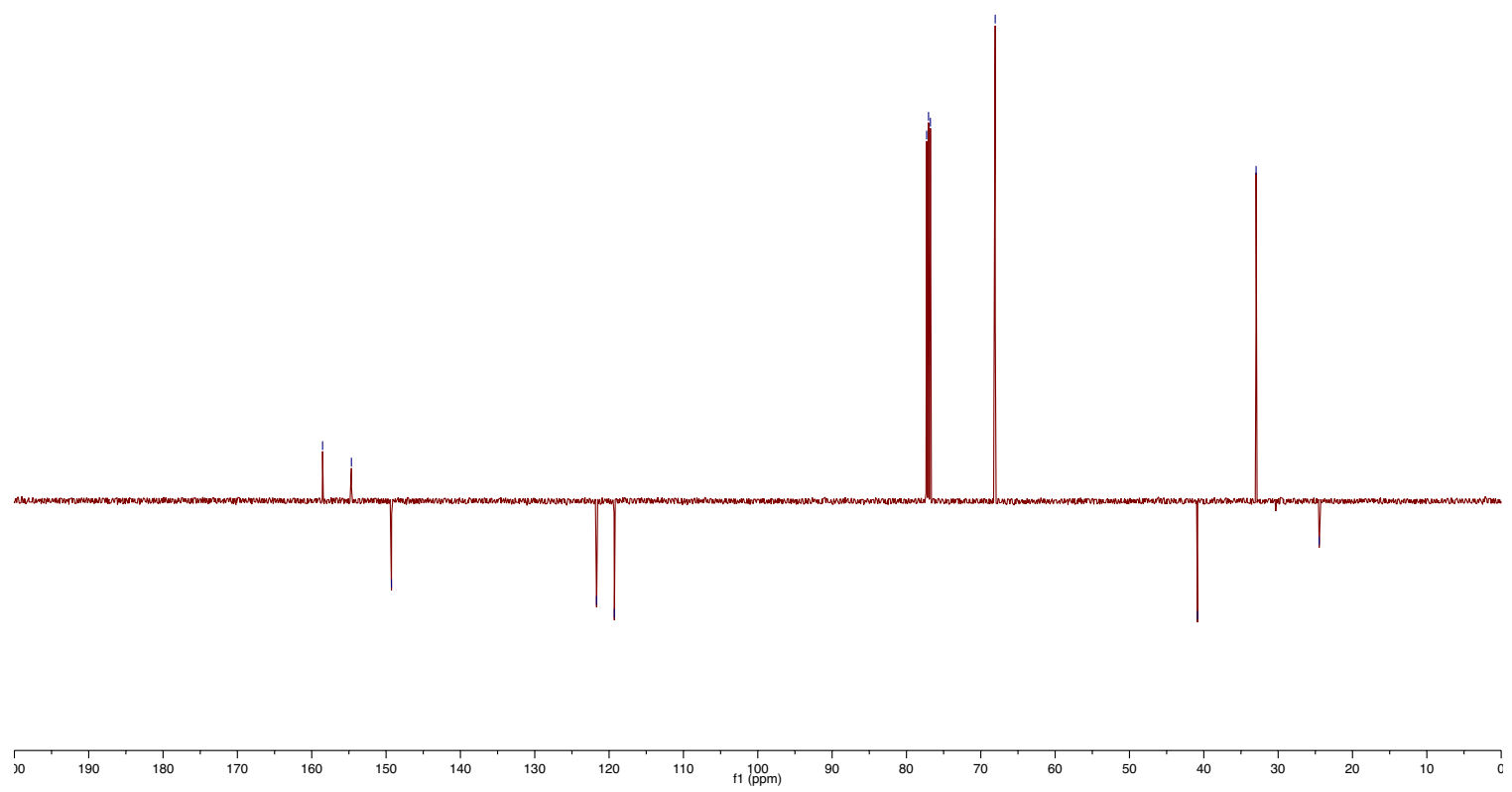


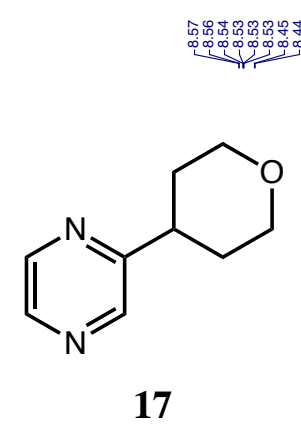

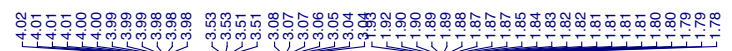
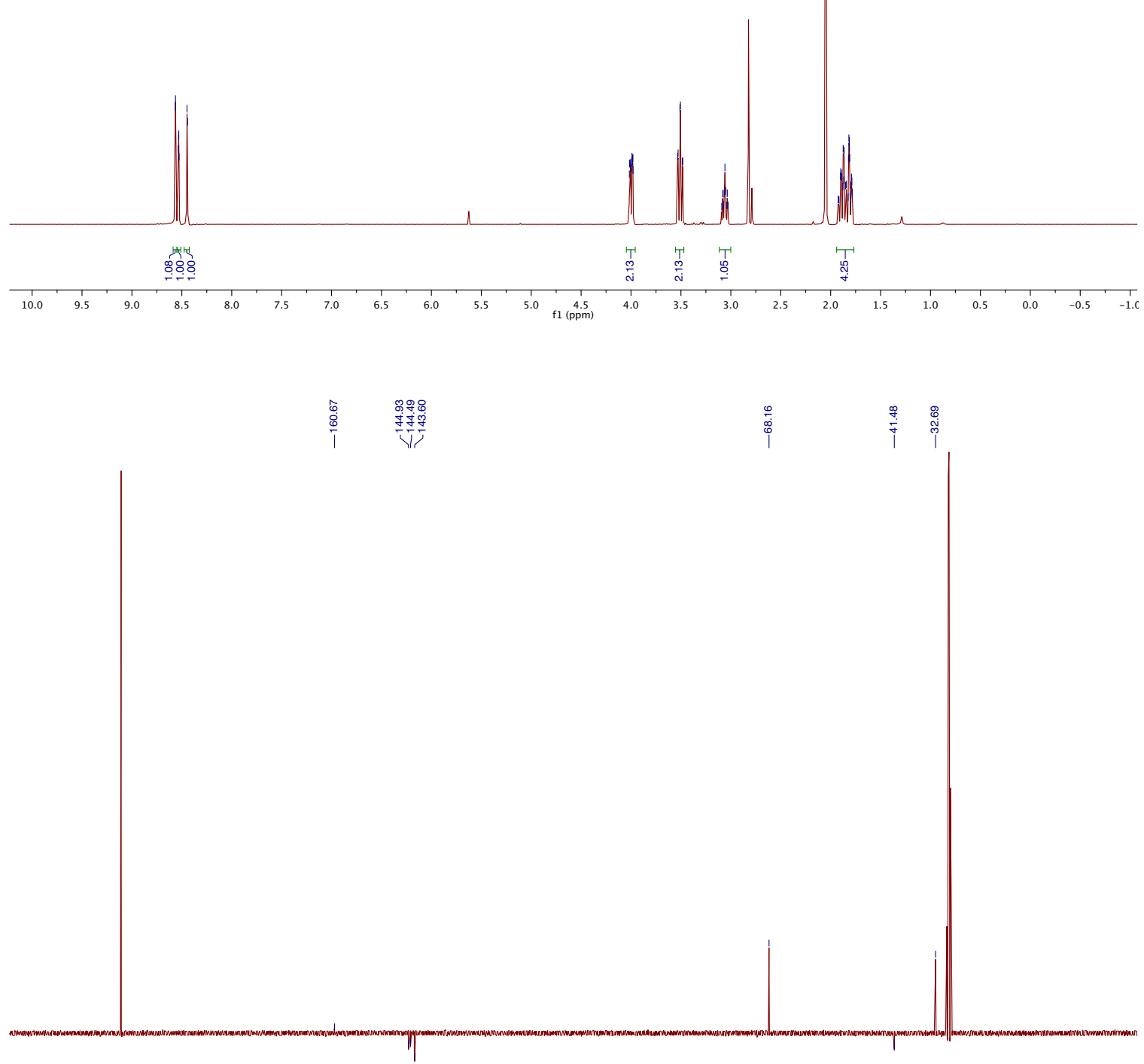

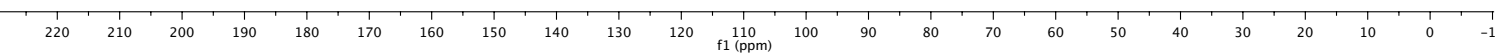



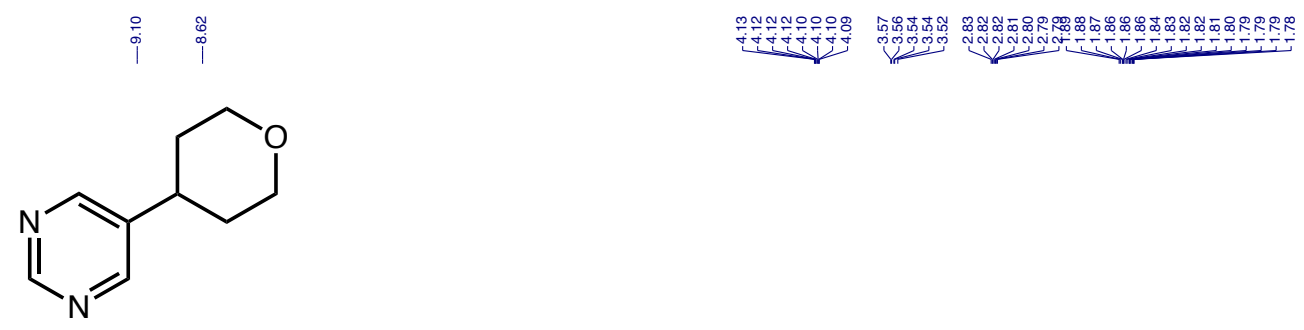

18
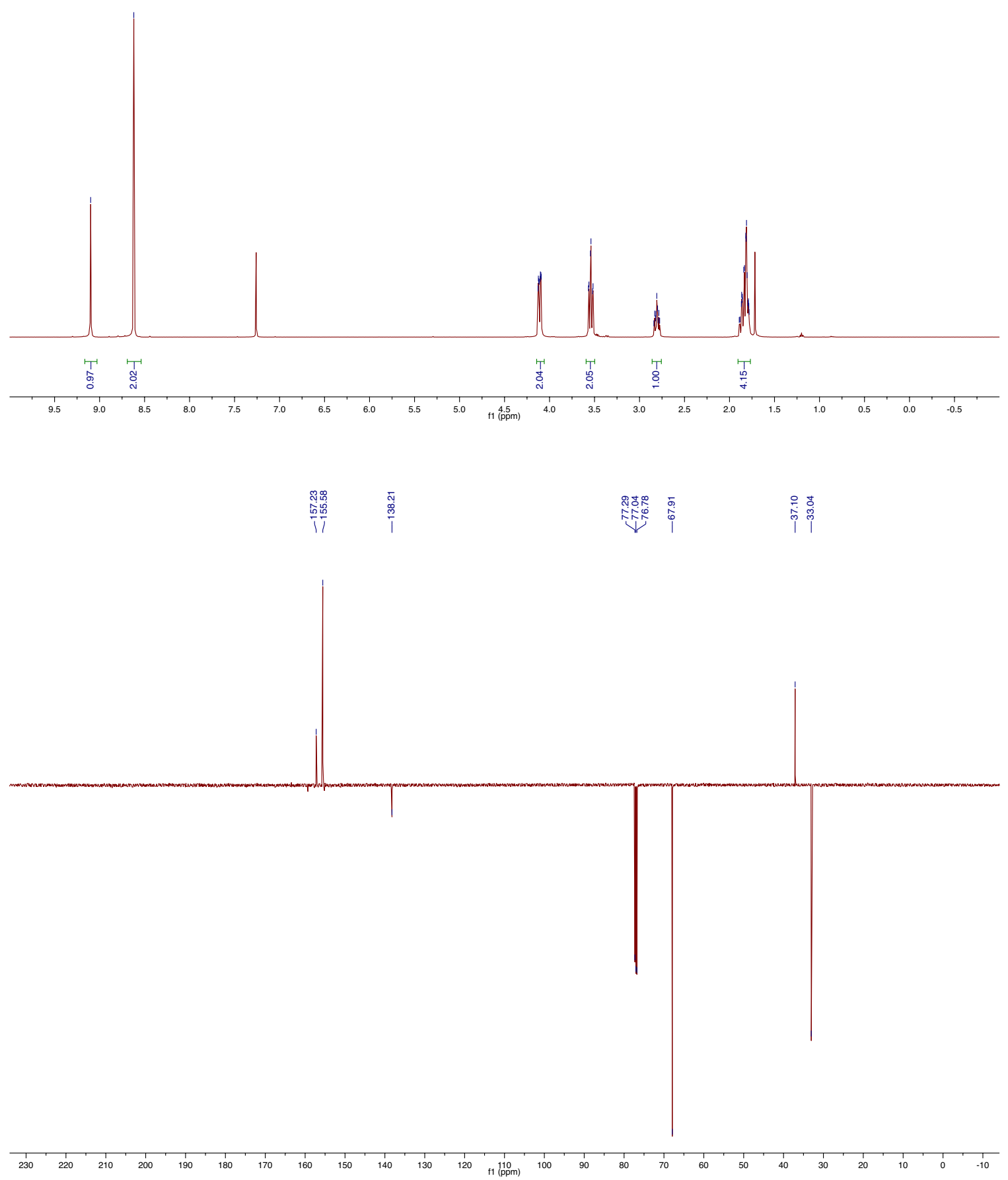

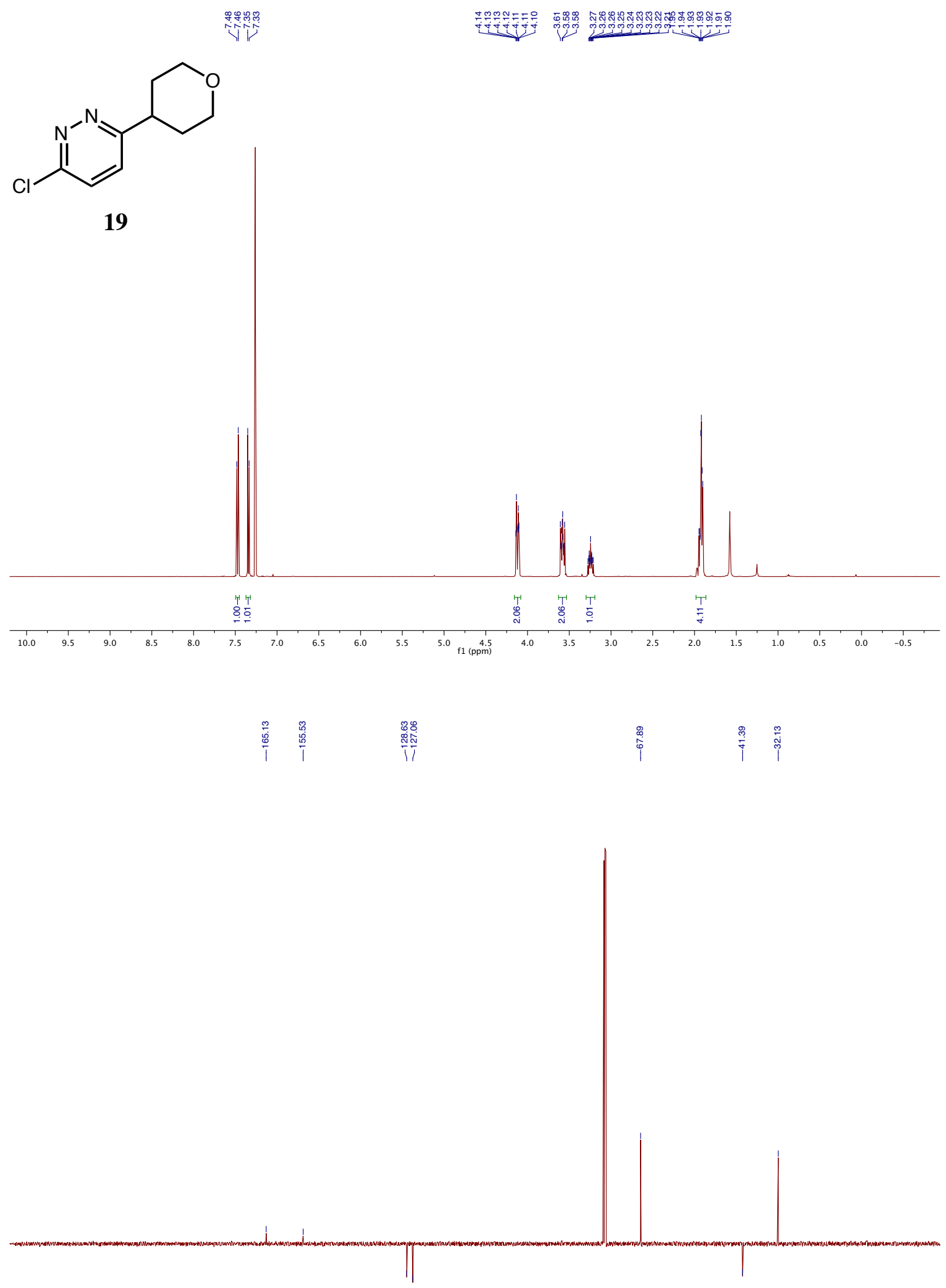

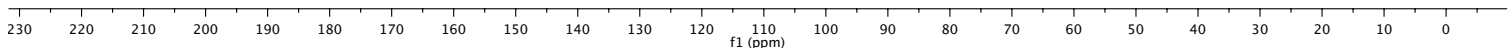




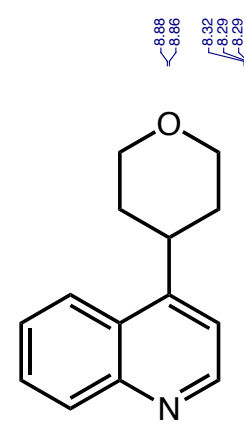

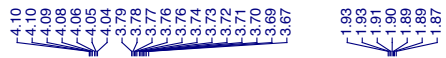

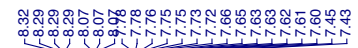

20

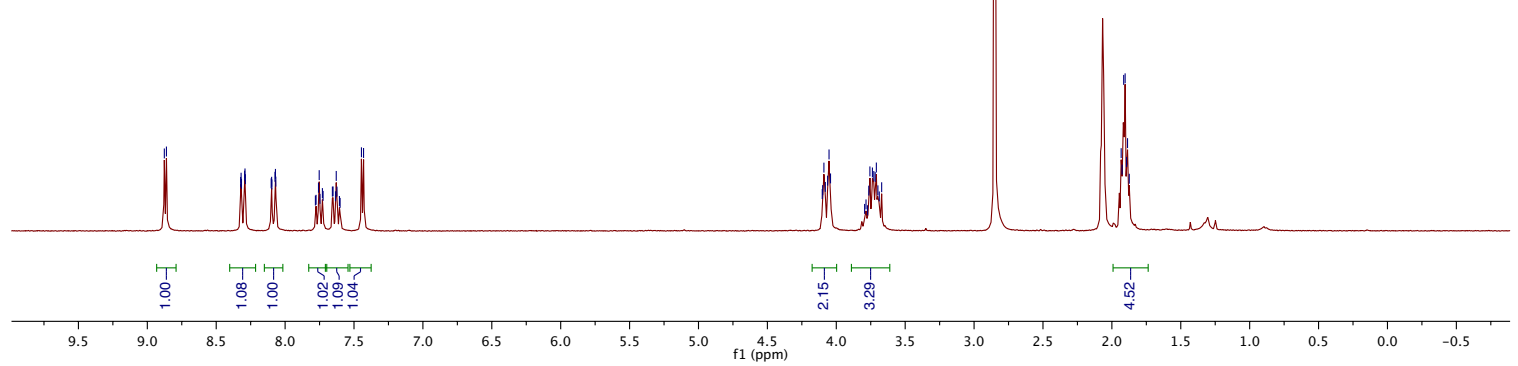

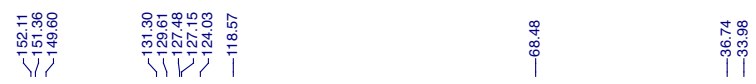

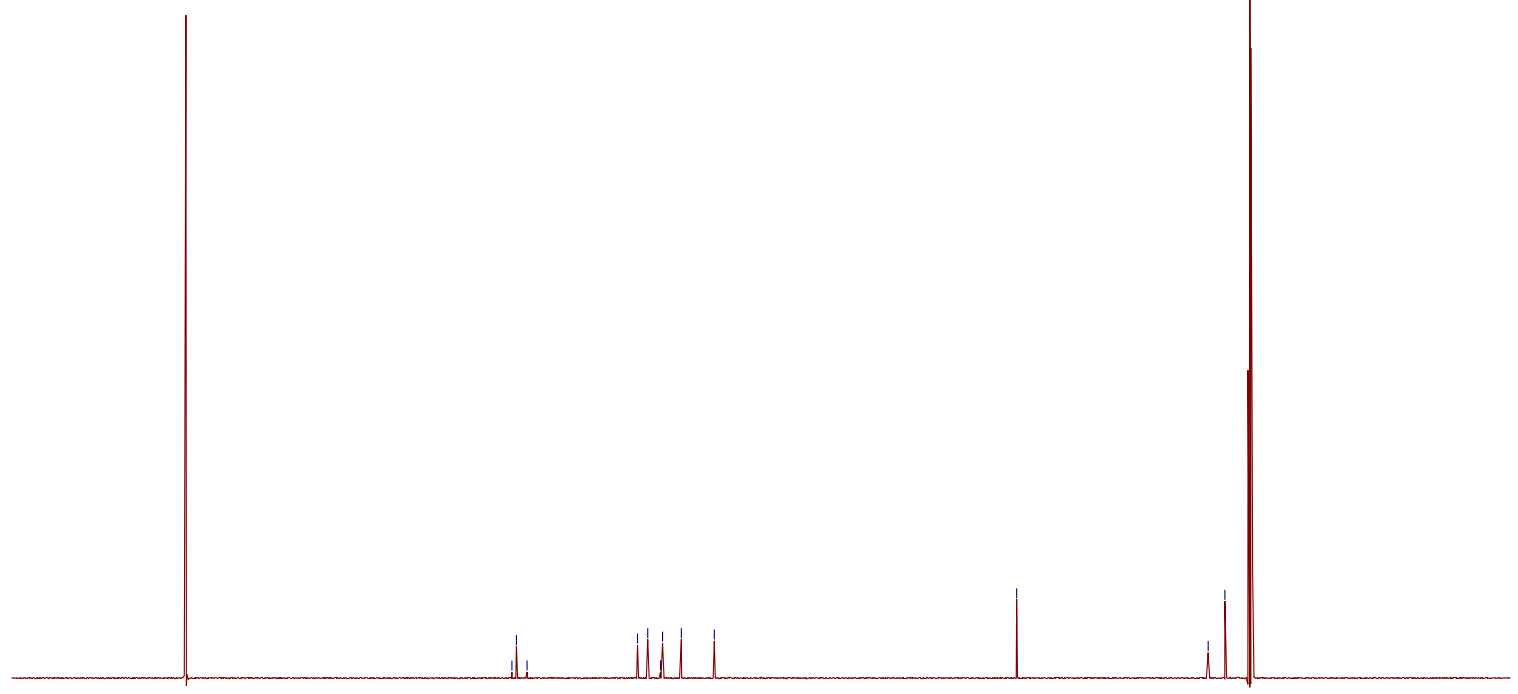

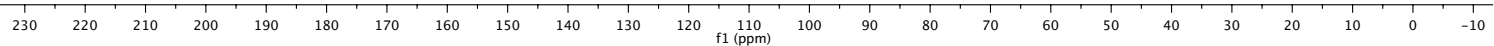



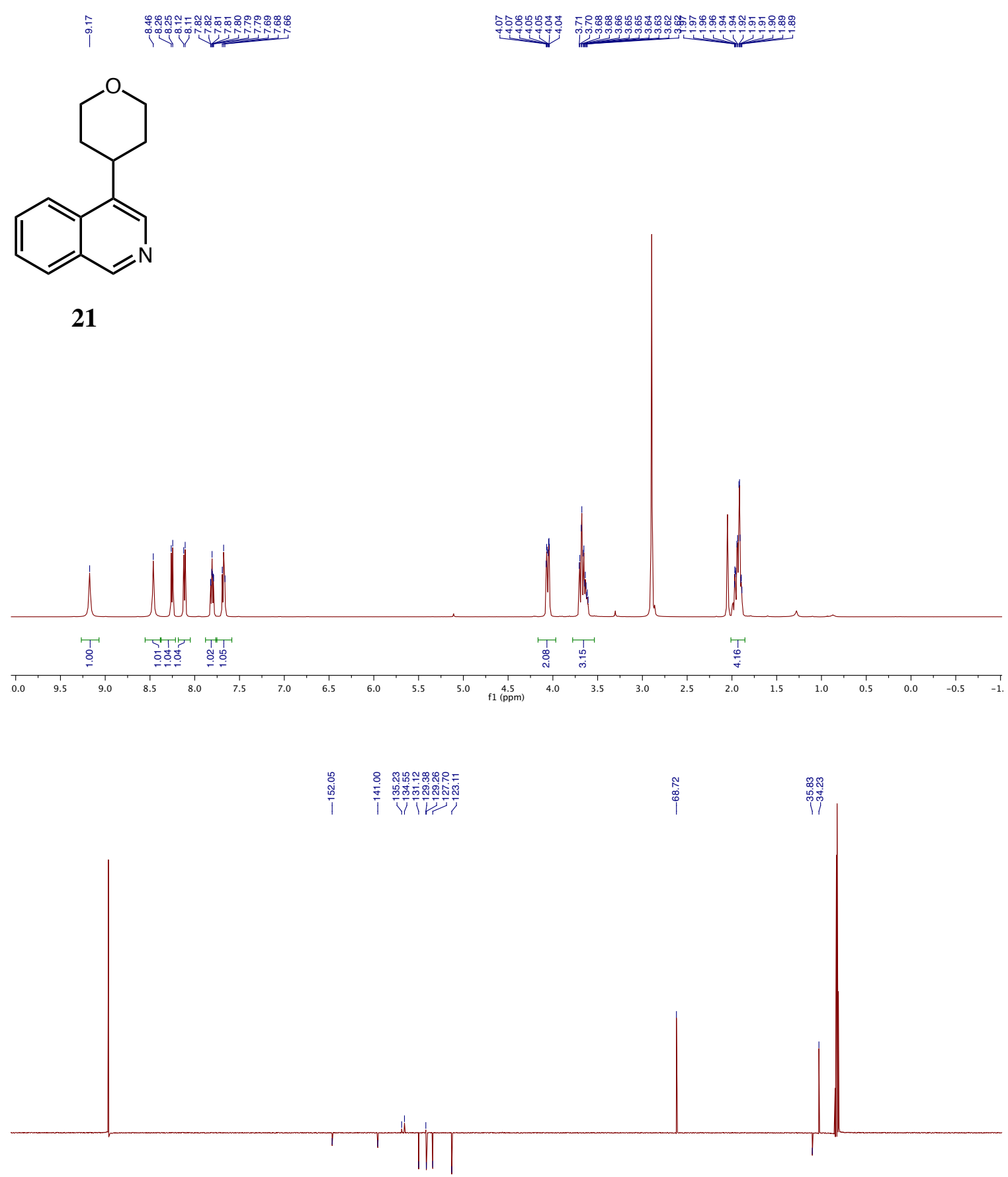

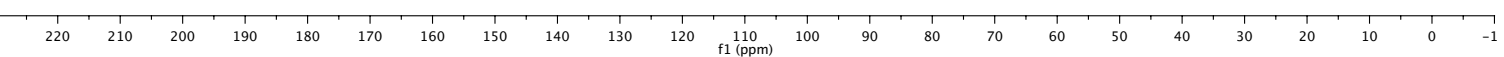




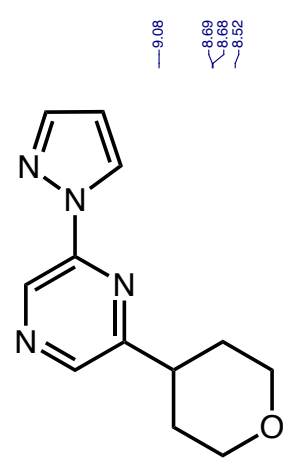

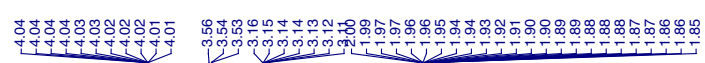

22
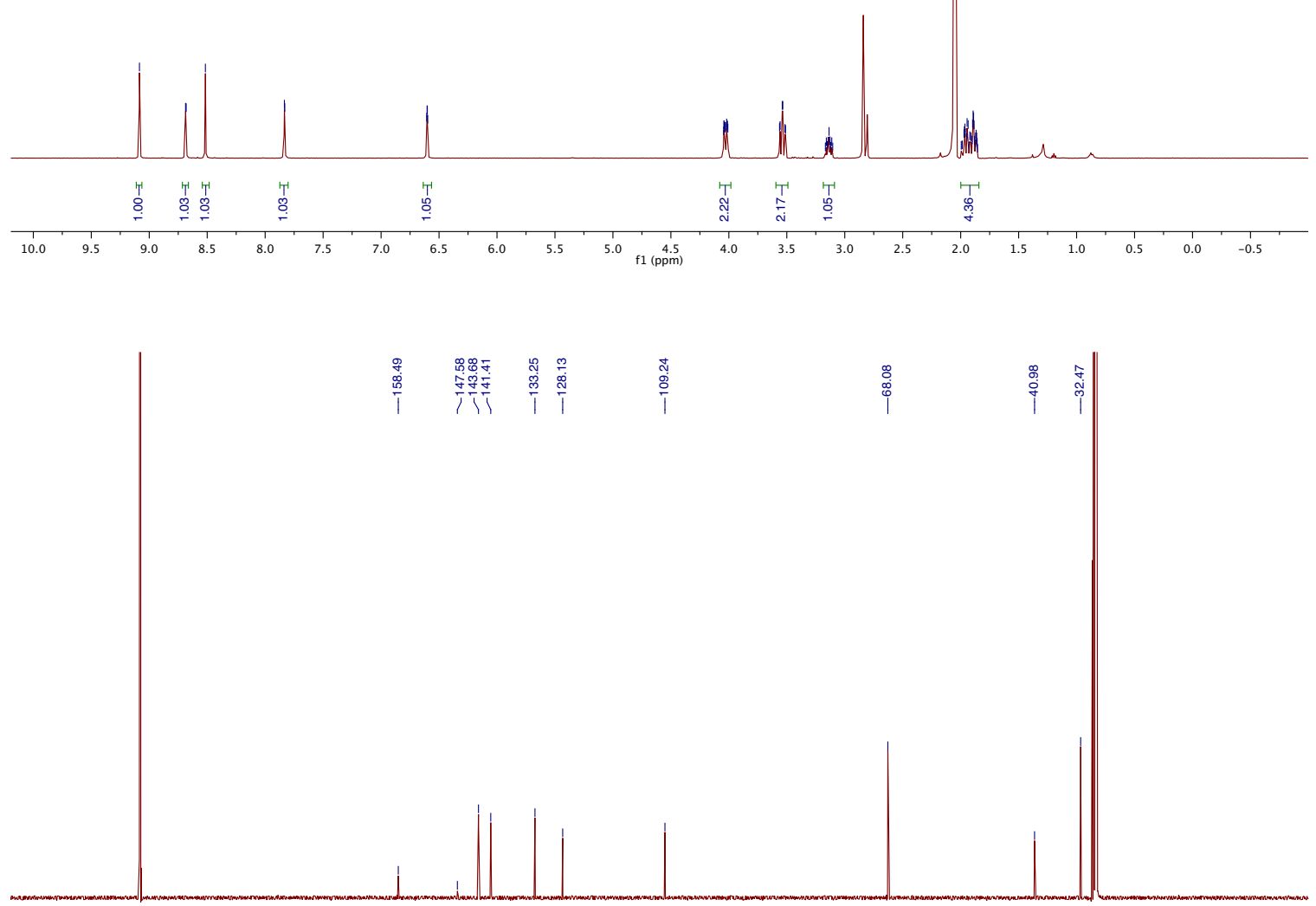

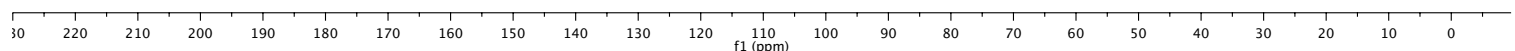




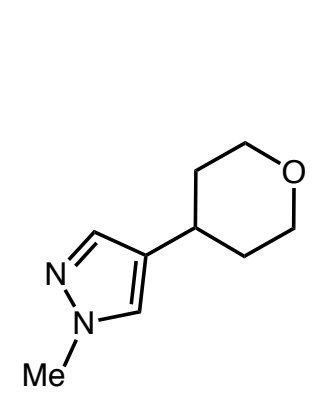

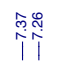

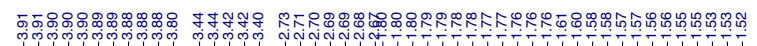

23
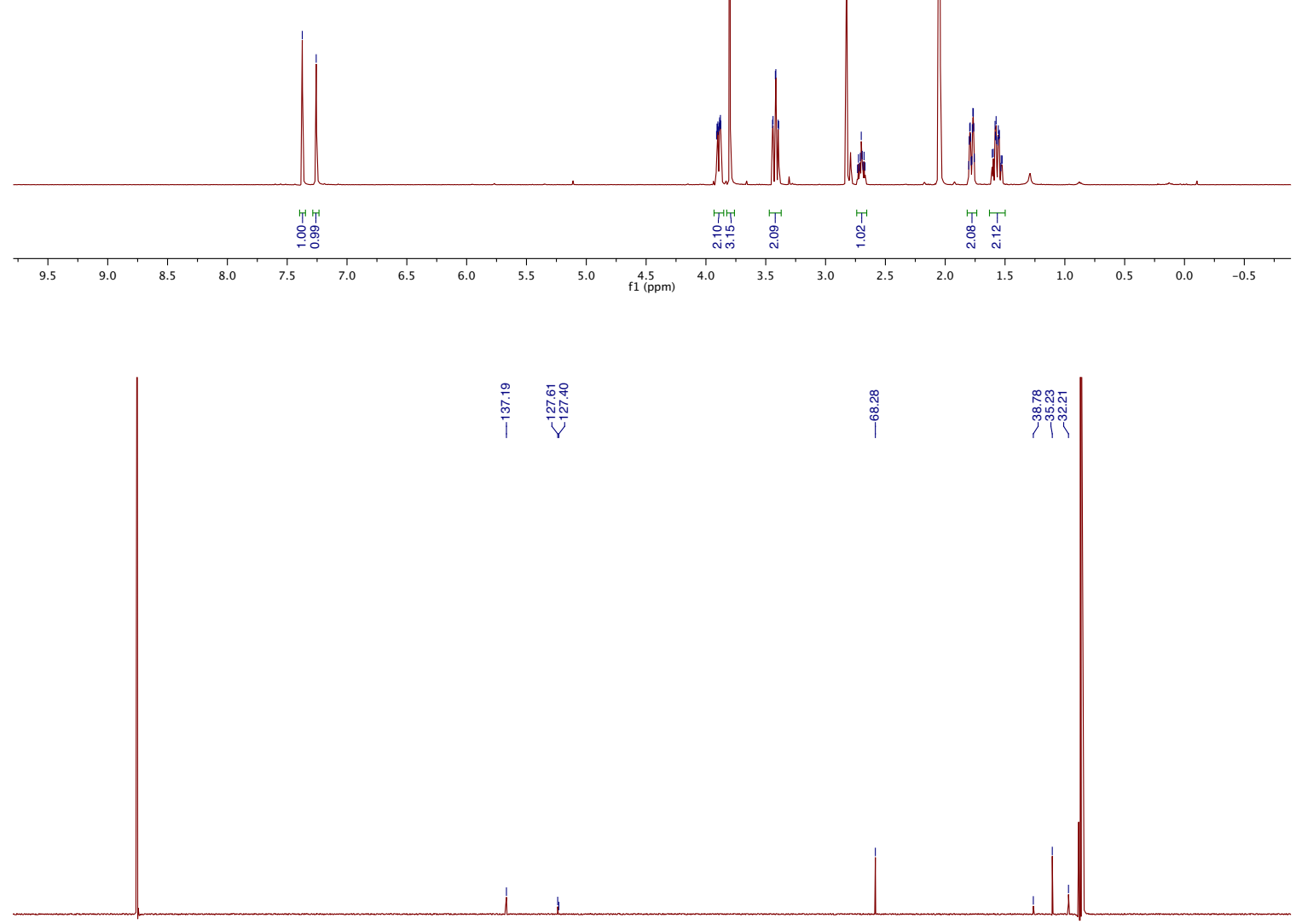

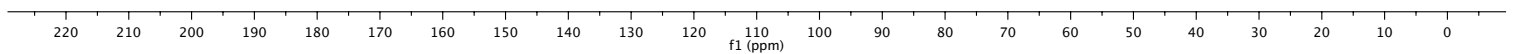



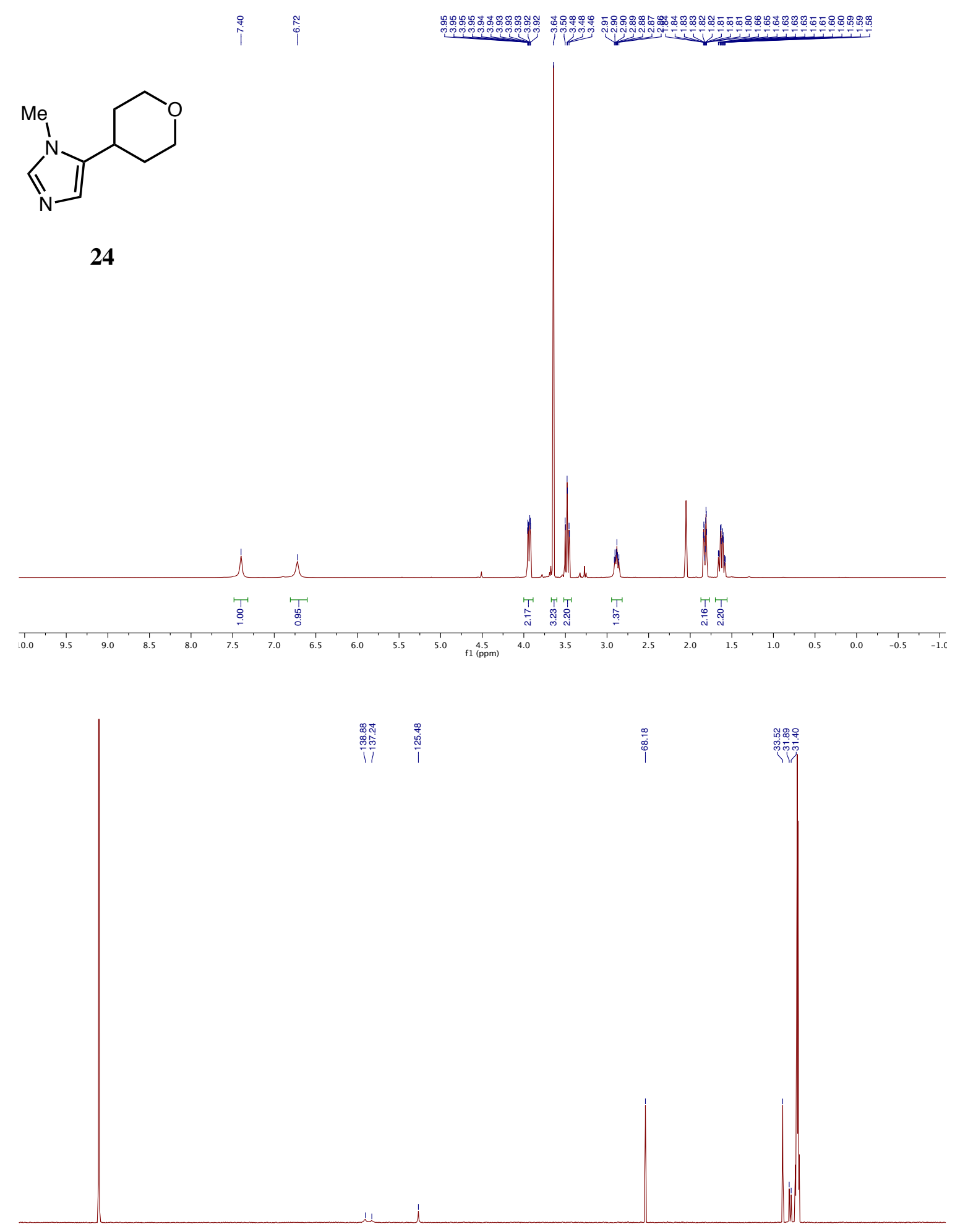

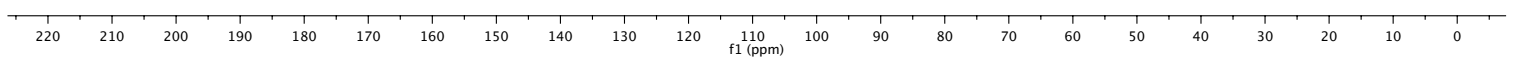




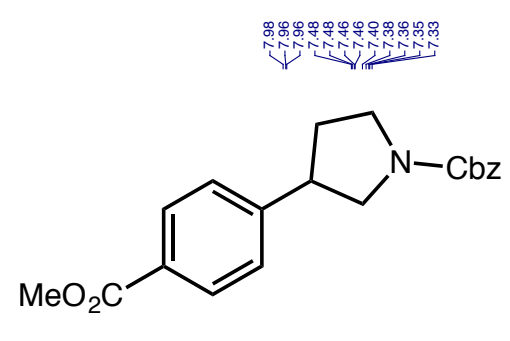

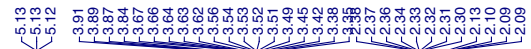

27
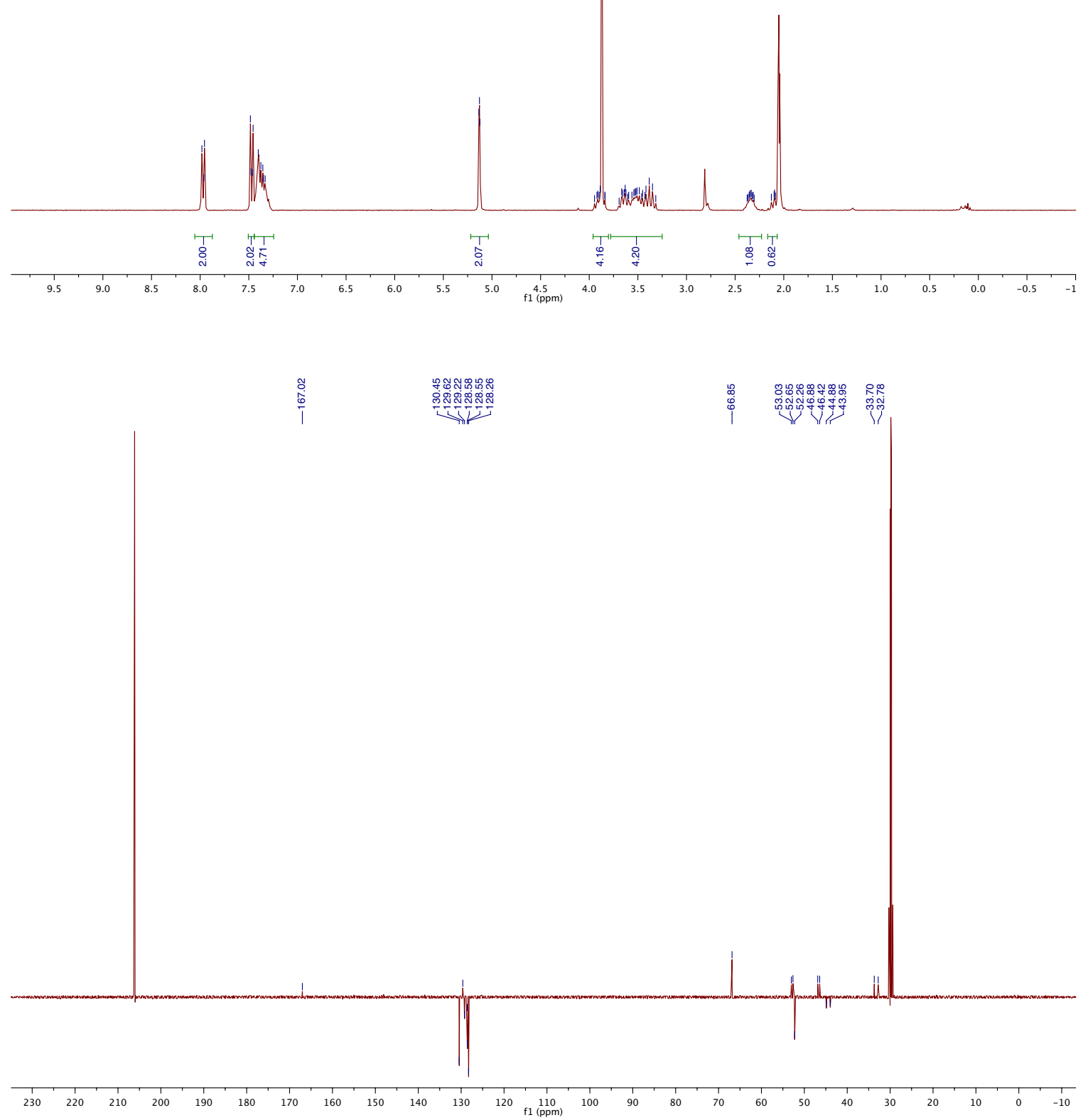


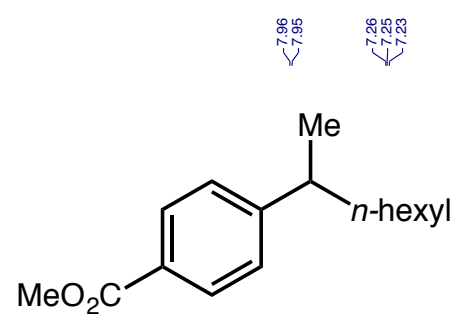

28

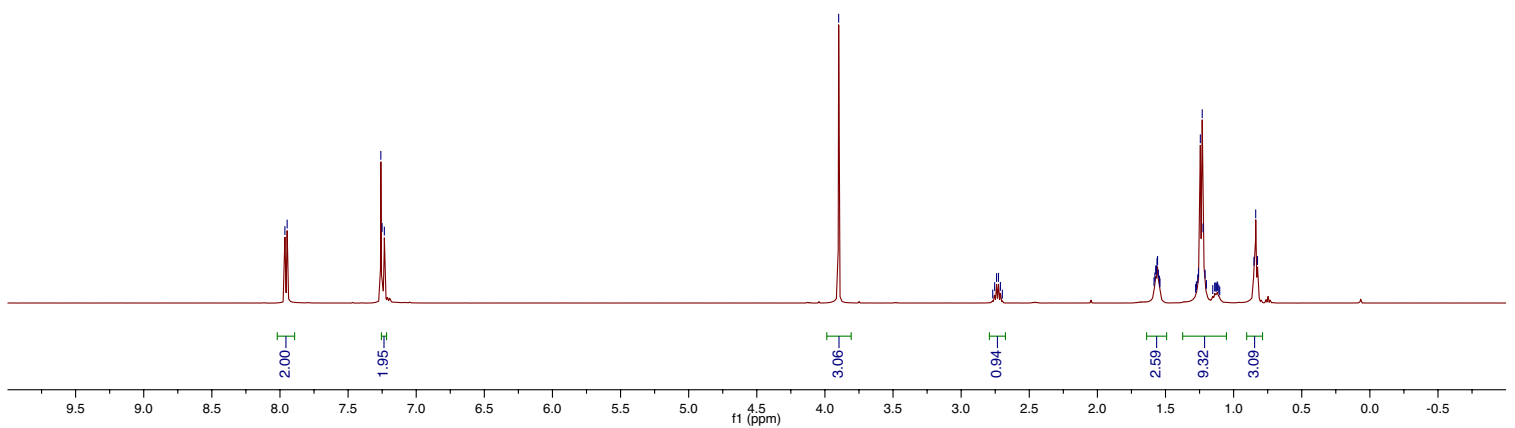

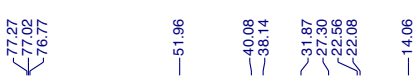

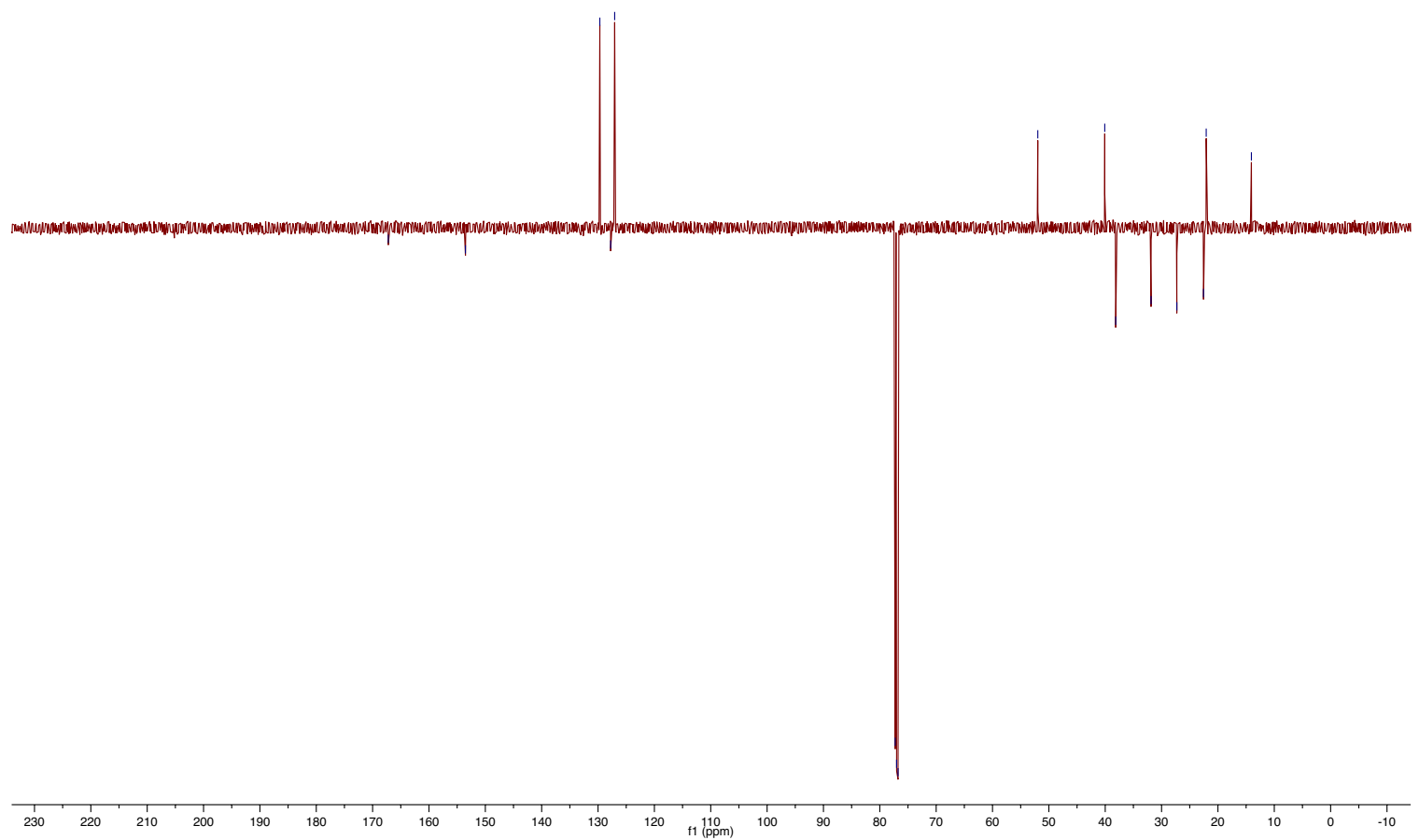




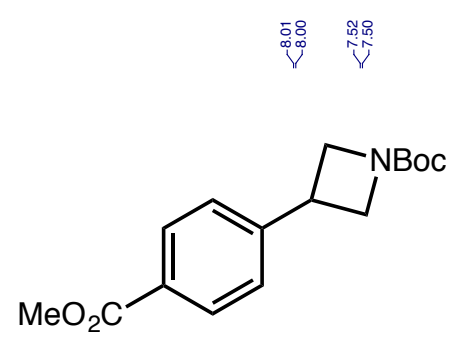

身

$\stackrel{+}{i}$

31
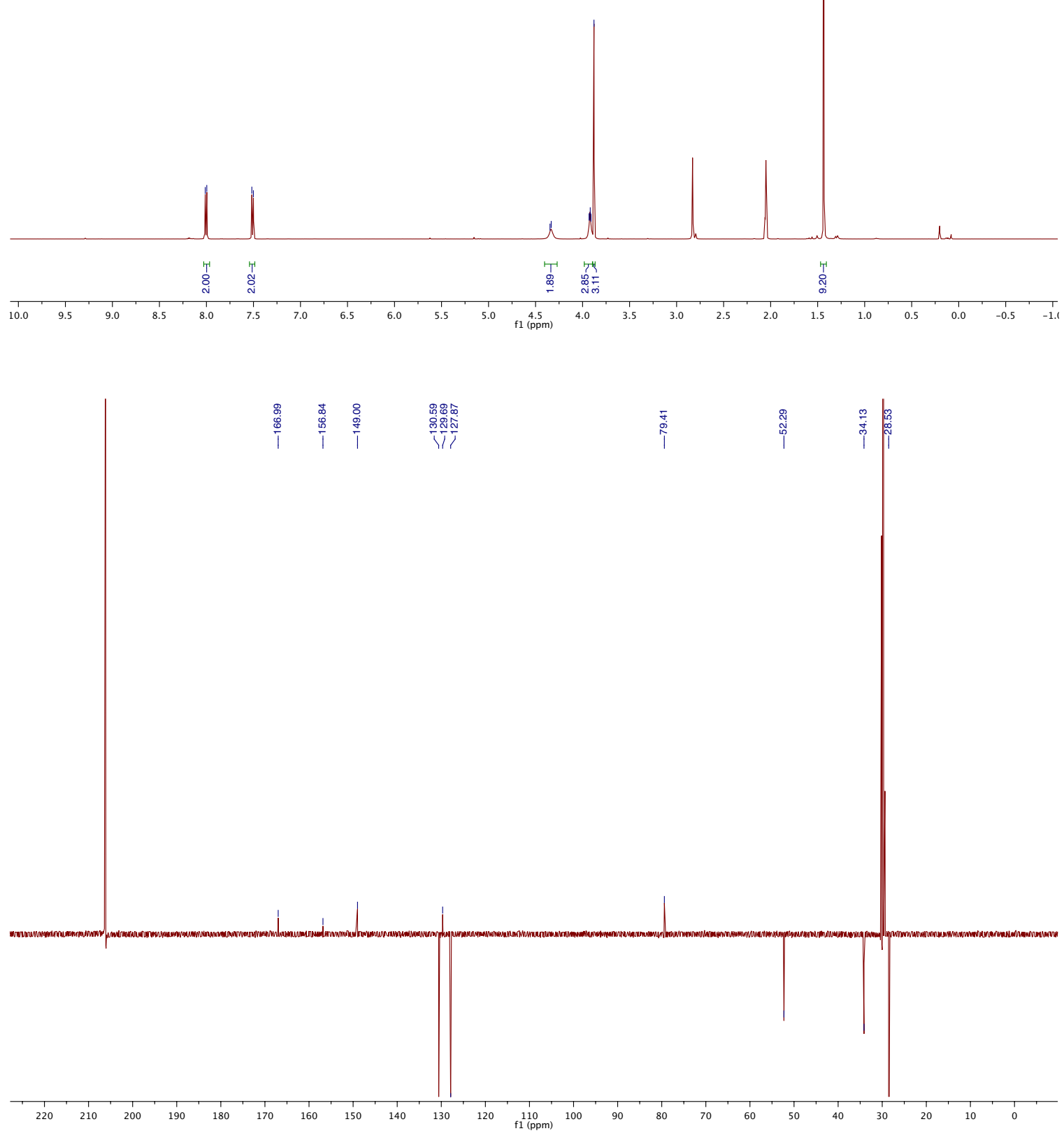


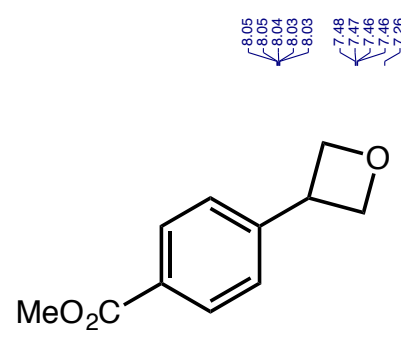

32

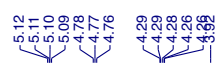

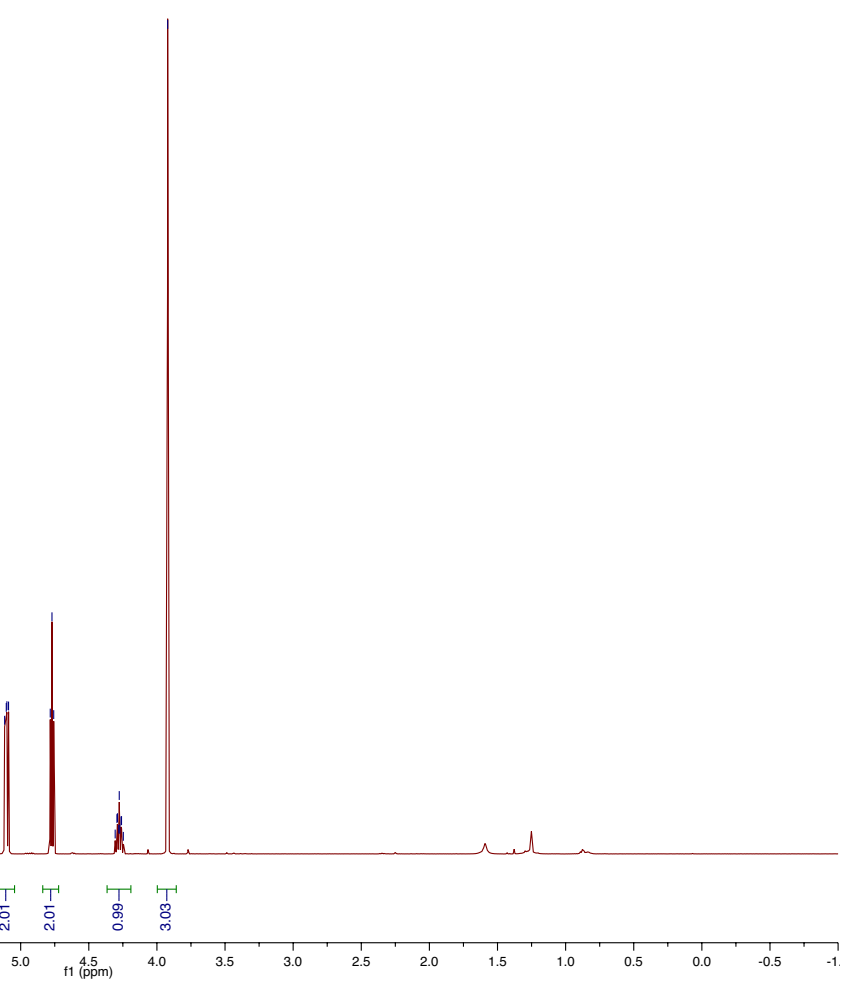

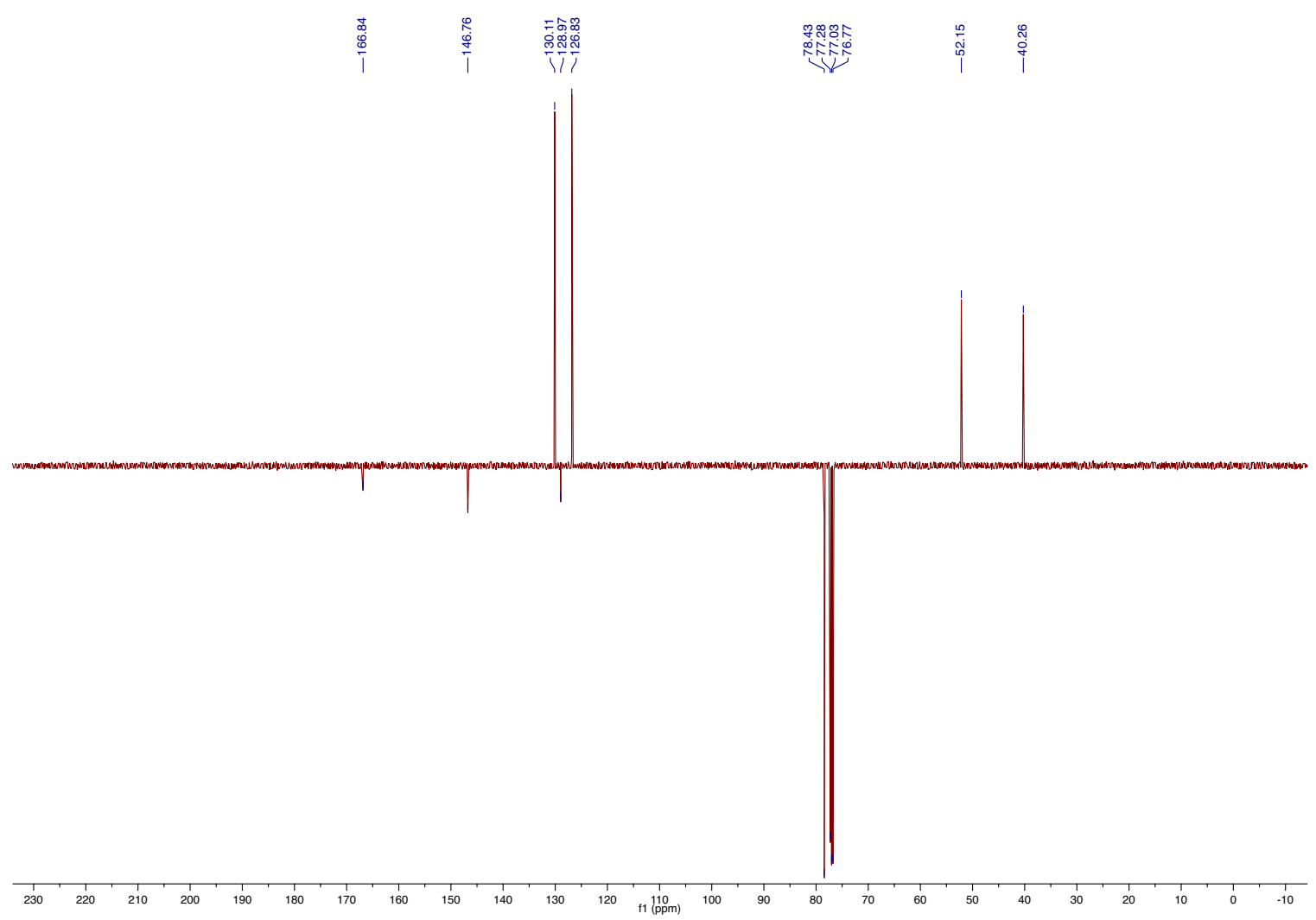


<smiles>COC(=O)c1ccc(CCCCC#N)cc1</smiles>

33
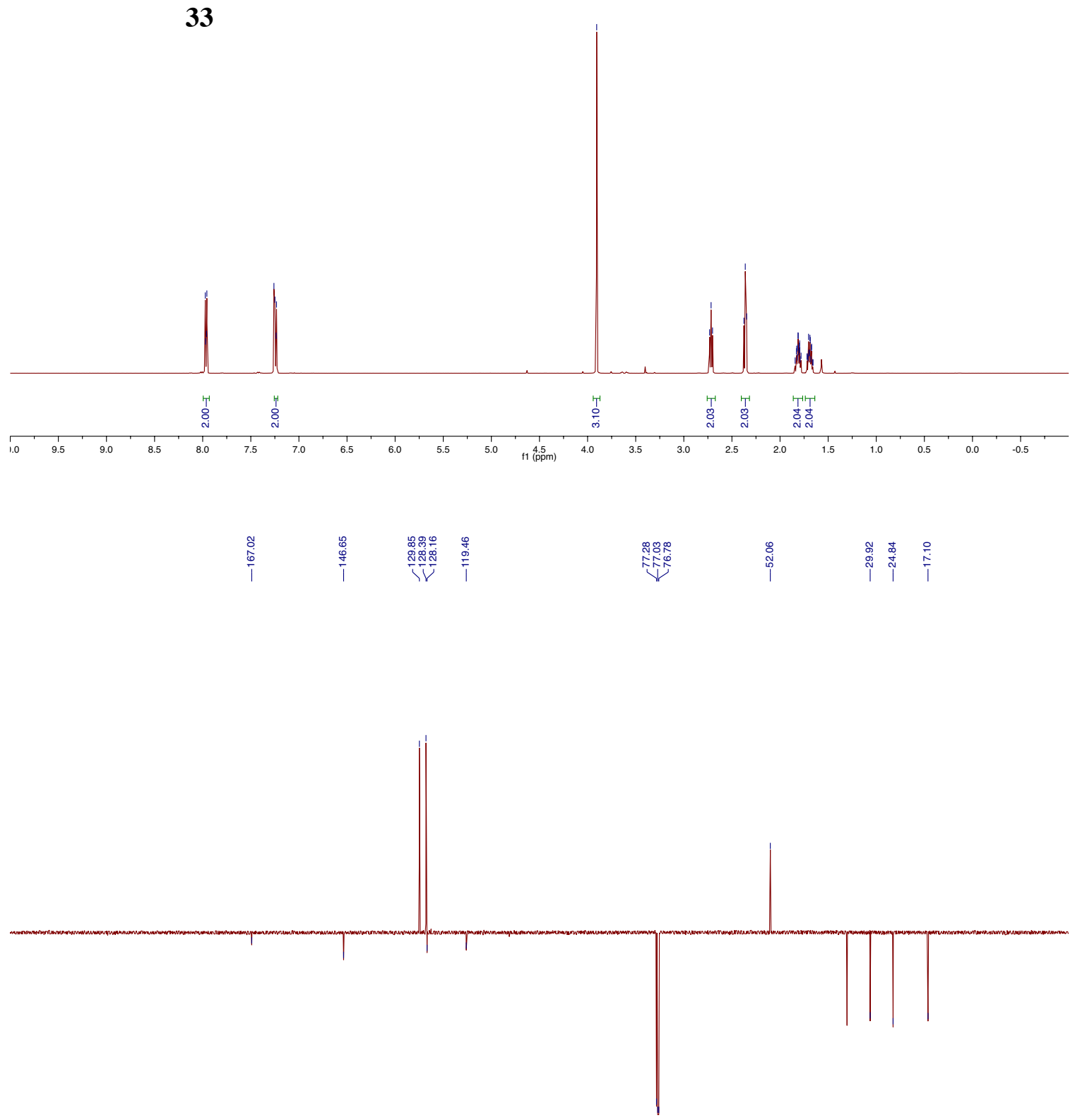

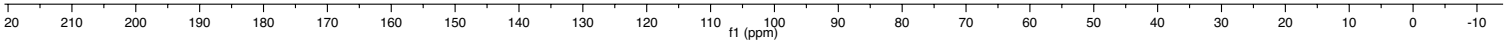




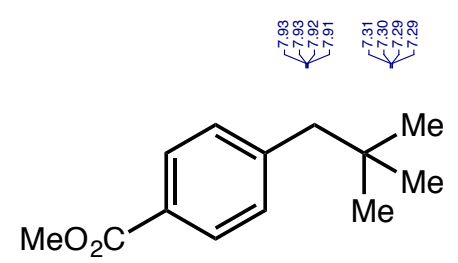

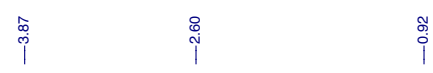

36
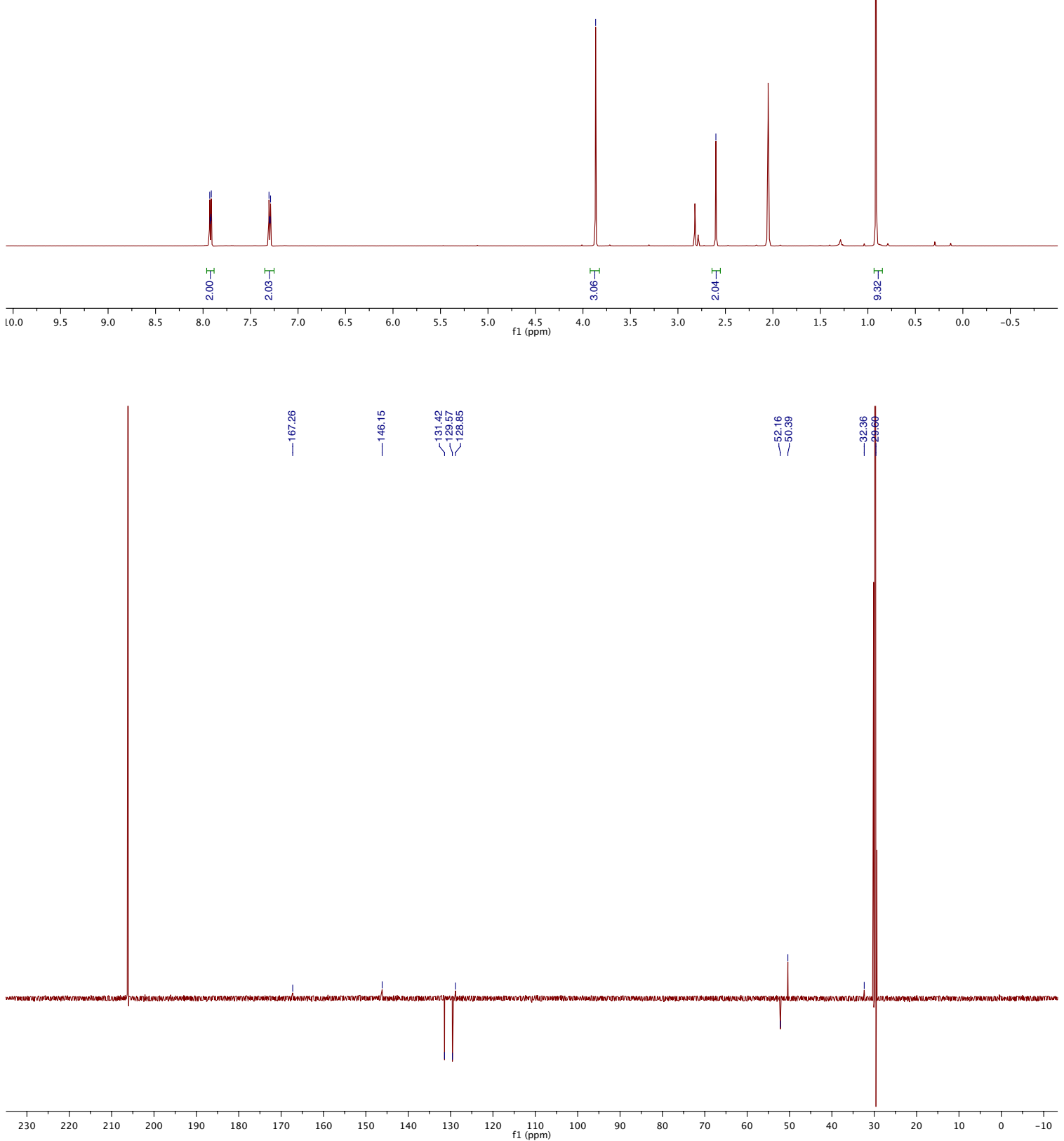